\title{
Ni-Catalyzed Intermolecular Allylic Dearomatization Reaction of Tryptophols and Tryptamines
}

\author{
Hui-Jun Zhang ${ }^{\dagger}$, Qing Gu*, and Shu-Li You*,†,* \\ ${ }^{\dagger}$ School of Chemistry and Molecular Engineering, East China University of Science \\ and Technology, 130 Meilong Road, Shanghai 200237, China \\ State Key Laboratory of Organometallic Chemistry, Shanghai Institute of Organic \\ Chemistry, Chinese Academy of Sciences, 345 Lingling Lu, Shanghai 200032, China \\ E-mail: $\underline{\text { slyou@ } \text { sioc.ac.cn }}$
}

\section{Table of Contents}

General methods S2

Optimization of the reaction conditions S3

Experimental details and characterization data S5

Transformation of products S25

X-Ray crystal data of $\mathbf{3 g}$ S28

Copies of NMR spectra S30

References 


\section{General methods}

Unless stated otherwise, all reactions were carried out in flame-dried glassware under a dry argon atmosphere. All solvents were purified and dried according to standard methods prior to use.

${ }^{1} \mathrm{H}$ and ${ }^{13} \mathrm{C}$ NMR spectra were recorded on an Agilent instrument $(400 \mathrm{MHz}$ and $100 \mathrm{MHz}$, respectively) or a Varian instrument (400 MHz and $100 \mathrm{MHz}$, respectively) or a Bruker instrument (400 $\mathrm{MHz}$ and $100 \mathrm{MHz}$, respectively) and internally referenced to tetramethylsilane signal or residual protio solvent signals. ${ }^{19} \mathrm{~F}$ NMR spectra were recorded on an Agilent instrument $(376 \mathrm{MHz})$ or a Bruker instrument (376 MHz) and referenced relative to $\mathrm{CFCl}_{3}$. Data for ${ }^{1} \mathrm{H} \mathrm{NMR}$ are recorded as follows: chemical shift $(\delta, \mathrm{ppm})$, multiplicity $(\mathrm{s}=$ singlet, $\mathrm{d}=$ doublet, $\mathrm{t}=$ triplet, $\mathrm{m}=$ multiplet or unresolved, br = broad singlet, coupling constant(s) in $\mathrm{Hz}$, integration). Data for ${ }^{13} \mathrm{C}$ NMR are reported in terms of chemical shift $(\delta, \mathrm{ppm})$.

Tryptophols $^{[1]}$, tryptamines ${ }^{[2]}$ and allylic alcohols ${ }^{[3]}$ were synthesized according to the literature procedure. 


\section{Optimization of the reaction conditions}

Table S1. Screening of chiral ligands. ${ }^{a} b c$

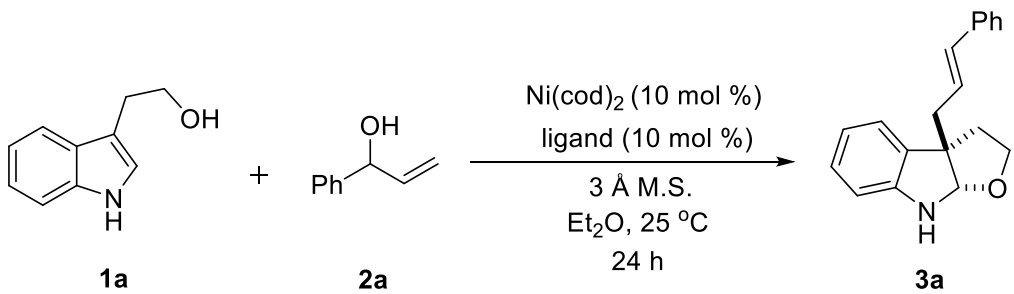<smiles>c1ccc(-c2ccc3ccccc3c2-c2c(-c3ccccc3)ccc3ccccc23)cc1</smiles>

L1

(R)-BINAP $80 \%$ NMR yield, $9 \%$ ee<smiles>c1ccc(-c2ccc3c(c2-c2c(P(c4ccccc4)c4ccccc4)ccc4c2CCCC4)CCCC3)cc1</smiles>

L2

(R)- $\mathrm{H}_{8}$-BINAP $78 \%$ NMR yield, $8 \%$ ee

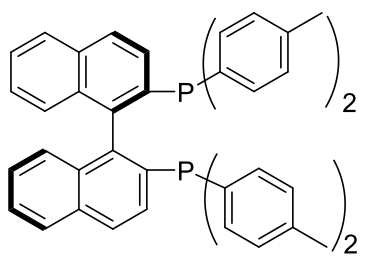

L3

(R)-TOL-BINAP $74 \%$ NMR yield, $1 \%$ ee<smiles>CN(C)p1oc2ccc3ccccc3c2c2c(ccc3ccccc32)o1</smiles>

(S)-L4

trace<smiles>C1=Cc2ccccc2N(p2oc3ccc4ccccc4c3c3c(ccc4ccccc43)o2)c2ccccc21</smiles>

L5

(S)-Carreira ligand trace<smiles>FC1(F)Oc2ccc(-c3ccccc3)c(-c3c(-c4ccccc4)ccc4c3OC(F)(F)O4)c2O1</smiles>

L10

(R)-DIFLUORPHOS $54 \%$ NMR yield, $9 \%$ ee

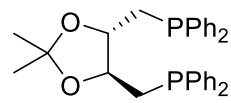

L14

(S,S)-DIOP trace<smiles>CC(C)(C)[C@H]1COC(c2ccc3ccccc3c2-c2c(P)ccc3ccccc23)=N1</smiles>

$\left(S, R_{a}\right)$-L6 trace<smiles>COc1cccc(P)c1-c1c(OC)cccc1-c1ccccc1</smiles>

L11 (R)-MeO-BIPHEP $80 \%$ NMR yield, $0 \%$ ee<smiles>c1ccc([C@@H]2N=C(CC3=N[C@H](c4ccccc4)[C@@H]([PH+](c4ccccc4)c4ccccc4)O3)O[C@H]2c2ccccc2)cc1</smiles>

$(R, R, R, R)-\mathbf{L} 15$ trace<smiles>CCCCc1ccc2c(c1-c1c([18F])ccc3c1OCO3)OCO2</smiles>

L7: $\mathrm{Ar}=\mathrm{Ph},(\mathrm{S})$-SEGPHOS $75 \%$ NMR yield, $14 \%$ ee L8: $\mathrm{Ar}=3,5-\mathrm{BBu}-4-\mathrm{MeOC}_{6} \mathrm{H}_{2}$, (S)-DTBM-SEGPHOS trace<smiles>C[C@@H]1CC[C@@H](C)P1c1ccccc1P1[C@H](C)CC[C@H]1C</smiles><smiles>O=C(N[C@H]1CCCC[C@@H]1NC(=O)c1ccccc1P)c1ccccc1P</smiles>

(S,S)-L13

$(R, R)$-Me-DUPHOS trace<smiles>CC(C)(C)[C@@H]1COC(c2ccccc2P)=N1</smiles>

(S)-L16 trace<smiles>Pc1ccccc1-c1c(-c2ccccc2)ccc2c1OCCO2</smiles>

19

(S)-SYNPHOS $44 \%$ NMR yield, $3 \%$ ee 


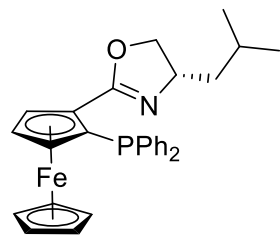

$\left(S, S_{p}\right)-\mathrm{L} 18$

$12 \%$ NMR yield, $67 \%$ ee

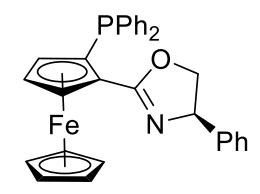

$\left(R, R_{p}\right)-\mathrm{L} 22$

$2 \%$ NMR yield, $85 \%$ ee

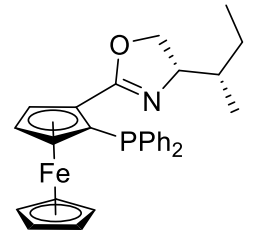

$\left(S, S, S_{p}\right)$-L19

$11 \%$ NMR yield, $67 \%$ ee

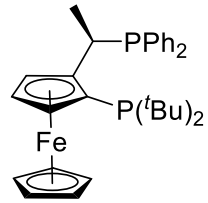

$\left(S, S_{p}\right)$-L23

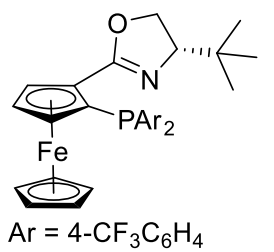

$\left(S, S_{p}\right)-\mathbf{L} 20$

$21 \%$ NMR yield, $16 \%$ ee

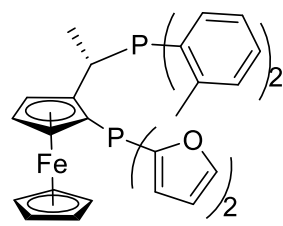

$\left(R, S_{p}\right)$-L24

$86 \%$ NMR yield, $-1 \%$ ee

${ }^{a}$ Reaction conditions: 1a $(0.2 \mathrm{mmol}), \mathbf{2 a}(0.24 \mathrm{mmol}), \mathrm{Ni}(\operatorname{cod})_{2}(10 \mathrm{~mol} \%)$, ligand $(10 \mathrm{~mol} \%), 3 \AA$ M.S. $(50 \mathrm{mg})$ in $\mathrm{Et}_{2} \mathrm{O}(1 \mathrm{~mL})$ at $25{ }^{\circ} \mathrm{C}$ for 24 h. ${ }^{b}$ Determined by ${ }^{1} \mathrm{H}$ NMR of the crude reaction mixture using dibromomethane as an internal standard. ${ }^{c}$ Determined by HPLC analysis.

Table S2. Screening of different molecular sieves. ${ }^{a}$<smiles>OCCc1c[nH]c2ccccc12</smiles>

$1 a$<smiles>C=CC(O)c1ccccc1</smiles>

$2 a$

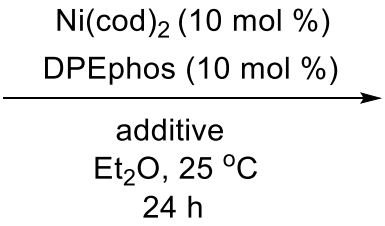

$24 \mathrm{~h}$<smiles>C(=C/c1ccccc1)\C[C@@]12CCO[C@@H]1Nc1ccccc12</smiles>

3a

\begin{tabular}{ccc}
\hline entry & additive & NMR yield $(\%)^{b}$ \\
\hline 1 & $3 \AA$ M.S. & $65\left(64^{c}\right)$ \\
2 & $4 \AA$ M.S. & $67\left(62^{c}\right)$ \\
3 & $5 \AA$ M.S. & 55
\end{tabular}

${ }^{a}$ Reaction conditions: 1a $(0.2 \mathrm{mmol}), 2 \mathrm{a}(0.24 \mathrm{mmol}), \mathrm{Ni}(\operatorname{cod})_{2}(10 \mathrm{~mol} \%)$, DPEphos (10 mol \%), $3 \AA$ M.S. $(50 \mathrm{mg})$ in $\mathrm{Et}_{2} \mathrm{O}(1 \mathrm{~mL})$ at $25{ }^{\circ} \mathrm{C}$ for 24 h. ${ }^{b}$ Determined by ${ }^{1} \mathrm{H}$ NMR of the crude reaction mixture using dibromomethane as an internal standard. ${ }^{c}$ Isolated yield. 


\section{Experimental details and characterization data}

\section{General procedure for $\mathrm{Ni}$-catalyzed tandem allylic substitution reaction}

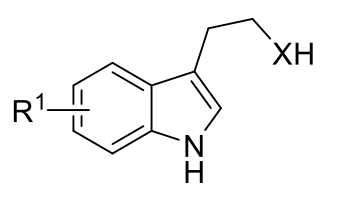

$\mathrm{X}=\mathrm{O}, \mathrm{NR}^{2}$<smiles>C=CC(O)Br</smiles>

2<smiles>[R]c1ccc2c(c1)NC([X])[C@]2(CC)C/C=C/Br</smiles>

3

In glovebox, $\mathrm{Ni}(\operatorname{cod})_{2}(0.026 \mathrm{mmol}, 13 \mathrm{~mol} \%)$ was added to a $10 \mathrm{~mL}$ vial. Then, DPEphos $(0.02 \mathrm{mmol}, 10 \mathrm{~mol} \%)$ and $\mathrm{Et}_{2} \mathrm{O}(1 \mathrm{~mL})$ were added to the vial under argon. The reaction mixture was stirred at room temperature. After 30 minutes, $3 \AA$ M.S. (50 $\mathrm{mg}), 1$ ( $0.2 \mathrm{mmol}, 1.0$ equiv) and 2 ( $0.24 \mathrm{mmol}, 1.2$ equiv) were added to the reaction vial under argon. After the reaction was complete (monitored by TLC), the reaction mixture was filtrated by silica gel and washed with EtOAc $(3 \times 15 \mathrm{~mL})$. The extracts were evaporated under reduced pressure. The diastereoisomeric ratio (3q(major) ${ }^{-}$ $3 \mathbf{q}_{\text {(minor) }}, 3 \mathbf{r}_{\text {(major) }}-3 \mathbf{r}_{\text {(minor) }}$ ) was determined by ${ }^{1} \mathrm{H}$ NMR of the crude reaction mixture. The residue was purified by silica gel column chromatography $(\mathrm{PE} / \mathrm{EtOAc}=20 / 1)$ to afford the desired product $\mathbf{3}$. The analytical data of the products are summarized below.<smiles>C(=C/c1ccccc1)\C[C@@]12CCO[C@@H]1Nc1ccccc12</smiles>

3a, yellow oil (Known compound, see: Kumar, N.; Maity, A.; Gavit, V. R.; Bisai, A. Chem. Commun. 2018, 54, 9083). $47.4 \mathrm{mg}, 85 \%$ yield. ${ }^{1} \mathrm{H}$ NMR (400 MHz, $\left.\mathrm{CDCl}_{3}\right) \delta$ 7.31-7.25 (m, 4H), 7.24-7.17 (m, 1H), 7.12-7.04 (m, 2H), $6.75(\mathrm{td}, J=7.2,0.8 \mathrm{~Hz}$, 1H), $6.58(\mathrm{~d}, J=8.0 \mathrm{~Hz}, 1 \mathrm{H}), 6.44(\mathrm{~d}, J=16.0 \mathrm{~Hz}, 1 \mathrm{H}), 6.11$ (ddd, $J=15.2,8.4,6.8$ $\mathrm{Hz}, 1 \mathrm{H}), 5.41$ (s, 1H), 4.60 (br s, 1H), 3.97 (ddd, $J=8.4,6.8,1.6 \mathrm{~Hz}, 1 \mathrm{H}), 3.57$ (ddd, 
$J=10.8,8.8,5.2 \mathrm{~Hz}, 1 \mathrm{H}), 2.75(\mathrm{ddd}, J=14.0,6.4,1.6 \mathrm{~Hz}, 1 \mathrm{H}), 2.69-2.57(\mathrm{~m}, 1 \mathrm{H})$, 2.27-2.09 (m, 2H).

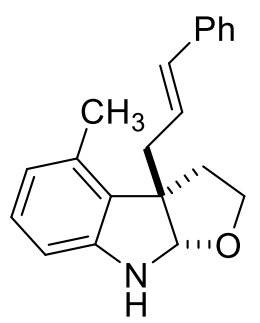

3b, yellow oil. $38.9 \mathrm{mg}, 67 \%$ yield. ${ }^{1} \mathrm{H}$ NMR (400 MHz, $\left.\mathrm{CDCl}_{3}\right) \delta$ 7.30-7.19 (m, 4H), 7.19-7.13 (m, 1H), 6.96 (t, $J=7.6 \mathrm{~Hz}, 1 \mathrm{H}), 6.53$ (d, $J=7.6 \mathrm{~Hz}, 1 \mathrm{H}), 6.50-6.36$ (m, 2H), 5.92 (ddd, $J=15.2,8.4,6.4 \mathrm{~Hz}, 1 \mathrm{H}), 5.42$ (s, 1H), 4.58 (br s, 1H), 3.99-3.88 (m, $1 \mathrm{H}), 3.63(\mathrm{td}, J=9.6,5.2 \mathrm{~Hz}, 1 \mathrm{H}), 2.98(\mathrm{dd}, J=14.4,6.0 \mathrm{~Hz}, 1 \mathrm{H}), 2.64(\mathrm{dd}, J=14.4$, $8.4 \mathrm{~Hz}, 1 \mathrm{H}), 2.36$ (s, 3H), $2.32(\mathrm{ddd}, J=12.4,5.6,2.4 \mathrm{~Hz}, 1 \mathrm{H}), 2.23-2.06(\mathrm{~m}, 1 \mathrm{H})$. ${ }^{13} \mathrm{C} \mathrm{NMR}\left(100 \mathrm{MHz}, \mathrm{CDCl}_{3}\right) \delta 150.0,137.4,134.0,132.8,128.9,128.6,128.4,127.3$, 126.3, 126.2, 121.4, 106.3, 97.6, 66.8, 58.2, 40.3, 38.1, 18.5. IR (thin film): $v_{\max }\left(\mathrm{cm}^{-1}\right)$ $=3349,3080,3054,3054,3025,2921,2862$, 2326, 2241, 2088, 1945, 1878, 1805, 1718, 1683, 1593, 1491, 1465, 1376, 1360, 1332, 1307, 1263, 1224, 1177, 1157, 1115, 1072, 1027, 962, 911, 873, 771, 739, 692, 627, 605, 577, 539, 509, 486, 419. HRMSESI calcd for $\mathrm{C}_{20} \mathrm{H}_{22} \mathrm{NO}[\mathrm{M}+\mathrm{H}]^{+}:$292.1696. Found: 292.1695.

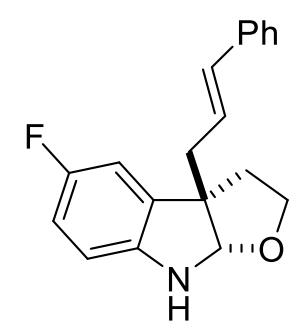

3c, yellow oil. $34.9 \mathrm{mg}, 59 \%$ yield. ${ }^{1} \mathrm{H}$ NMR $\left(400 \mathrm{MHz}, \mathrm{CDCl}_{3}\right) \delta$ 7.32-7.27 (m, 4H), 7.24-7.18 (m, 1H), $6.82(\mathrm{dd}, J=8.4,2.8 \mathrm{~Hz}, 1 \mathrm{H}), 6.80-6.74(\mathrm{~m}, 1 \mathrm{H}), 6.49(\mathrm{dd}, J=8.4$, $4.0 \mathrm{~Hz}, 1 \mathrm{H}), 6.47-6.41(\mathrm{~m}, 1 \mathrm{H}), 6.13-6.00(\mathrm{~m}, 1 \mathrm{H}), 5.42$ (s, 1H), 4.43 (br s, 1H), 3.97 (ddd, $J=8.8,7.2,1.6 \mathrm{~Hz}, 1 \mathrm{H}), 3.57$ (ddd, $J=10.8,8.8,5.2 \mathrm{~Hz}, 1 \mathrm{H}), 2.74$ (ddd, $J=$ 14.0, 6.4, $1.2 \mathrm{~Hz}, 1 \mathrm{H}), 2.61$ (ddd, $J=13.6,8.0,1.2 \mathrm{~Hz}, 1 \mathrm{H}), 2.26-2.16(\mathrm{~m}, 1 \mathrm{H}), 2.12$ (ddd, $J=12.0,5.2,1.6 \mathrm{~Hz}, 1 \mathrm{H}) .{ }^{19} \mathrm{~F} \mathrm{NMR}\left(376 \mathrm{MHz}, \mathrm{CDCl}_{3}\right) \delta-125.8(\mathrm{~m}) .{ }^{13} \mathrm{C} \mathrm{NMR}$ $\left(100 \mathrm{MHz}, \mathrm{CDCl}_{3}\right) \delta 157.2(\mathrm{~d}, J=235.5 \mathrm{~Hz}), 145.7,137.2,133.9(\mathrm{~d}, J=7.3 \mathrm{~Hz})$, 133.6, 128.7, 127.5, 126.3, 125.4, $114.5(\mathrm{~d}, J=23.3 \mathrm{~Hz}), 111.1(\mathrm{~d}, J=23.9 \mathrm{~Hz})$, 
$108.9(\mathrm{~d}, J=8.1 \mathrm{~Hz}), 98.4,67.3,58.5(\mathrm{~d}, J=1.8 \mathrm{~Hz}), 41.5,39.6$. IR (thin film): $v_{\max }\left(\mathrm{cm}^{-1}\right)=3350,3058,3026,2920,2868,2104,1946,1878,1721,1657,1613,1600$, 1485, 1448, 1360, 1334, 1275, 1254, 1233, 1182, 1146, 1114, 1012, 963, 909, 864, 806, 739, 693, 649, 572, 532, 502, 475. HRMS-ESI calcd for $\mathrm{C}_{19} \mathrm{H}_{19} \mathrm{FNO}[\mathrm{M}+\mathrm{H}]^{+}$: 296.1445. Found: 296.1442.

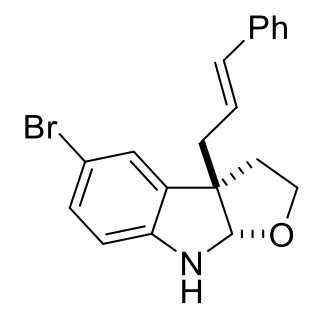

3d, yellow solid, m.p. $=105.2-106.0{ }^{\circ} \mathrm{C} .46 .2 \mathrm{mg}, 65 \%$ yield. ${ }^{1} \mathrm{H}$ NMR $(400 \mathrm{MHz}$, $\left.\mathrm{CDCl}_{3}\right) \delta 7.28(\mathrm{~d}, J=4.4 \mathrm{~Hz}, 4 \mathrm{H}), 7.24-7.18(\mathrm{~m}, 2 \mathrm{H}), 7.15(\mathrm{dd}, J=8.4,2.0 \mathrm{~Hz}, 1 \mathrm{H})$, 6.48-6.41 (m, 2H), 6.03 (ddd, $J=15.2,8.4,6.4 \mathrm{~Hz}, 1 \mathrm{H}), 5.40$ (s, 1H), 4.55 (br s, 1H), 4.08-3.88 (m, 1H), $3.56(\mathrm{ddd}, J=10.8,8.8,5.2 \mathrm{~Hz}, 1 \mathrm{H}), 2.74(\mathrm{ddd}, J=14.0,6.4,1.2$ $\mathrm{Hz}, 1 \mathrm{H}), 2.60$ (dd, $J=14.0,8.4 \mathrm{~Hz}, 1 \mathrm{H}), 2.19(\mathrm{td}, J=11.2,7.2 \mathrm{~Hz}, 1 \mathrm{H}), 2.14-2.08$ (m, 1H). ${ }^{13} \mathrm{C} \mathrm{NMR}\left(100 \mathrm{MHz}, \mathrm{CDCl}_{3}\right) \delta 148.7,137.2,134.6,133.7,131.0,128.6,127.5$, 126.7, 126.3, 125.4, 110.4, 109.7, 97.6, 67.3, 58.2, 41.5, 39.6. IR (thin film): $v_{\max }\left(\mathrm{cm}^{-}\right.$ $\left.{ }^{1}\right)=3333,3103,3058,3025,2970,2943,2921,2878,2404,2296,2083,1988,1941$, 1861, 1796, 1740, 1647, 1599, 1472, 1442, 1346, 1325, 1256, 1219, 1156, 1124, 1092, 1071, 1027, 1006, 964, 943, 915, 895, 876, 857, 839, 808, 739, 718, 690, 631, 601, 570, 550, 512, 464, 443, 425. HRMS-ESI calcd for $\mathrm{C}_{19} \mathrm{H}_{19}{ }^{79} \mathrm{BrNO}[\mathrm{M}+\mathrm{H}]^{+}: 356.0645$. Found: 356.0643.

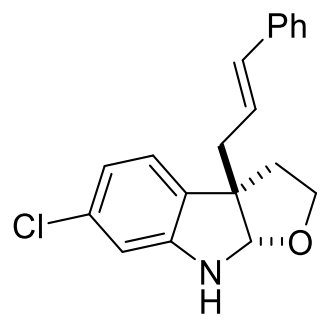

3e, brown solid, m.p. $=102.8-103.7{ }^{\circ} \mathrm{C} .29 .0 \mathrm{mg}, 47 \%$ yield. ${ }^{1} \mathrm{H}$ NMR $(400 \mathrm{MHz}$, $\left.\mathrm{CDCl}_{3}\right) \delta 7.29(\mathrm{~d}, J=4.0 \mathrm{~Hz}, 4 \mathrm{H}), 7.24-7.18(\mathrm{~m}, 1 \mathrm{H}), 6.98(\mathrm{~d}, J=7.6 \mathrm{~Hz}, 1 \mathrm{H}), 6.70$ $(\mathrm{dd}, J=7.6,1.6 \mathrm{~Hz}, 1 \mathrm{H}), 6.54(\mathrm{~d}, J=1.6 \mathrm{~Hz}, 1 \mathrm{H}), 6.44(\mathrm{~d}, J=16.0 \mathrm{~Hz}, 1 \mathrm{H}), 6.06$ (ddd, $J=15.6,8.4,6.4 \mathrm{~Hz}, 1 \mathrm{H}), 5.41$ (s, 1H), 4.66 (br s, 1H), 4.04-3.91 (m, 1H), 3.56 $(\mathrm{ddd}, J=11.2,8.8,5.2 \mathrm{~Hz}, 1 \mathrm{H}), 2.73(\mathrm{ddd}, J=14.0,6.4,0.8 \mathrm{~Hz}, 1 \mathrm{H}), 2.60(\mathrm{dd}, J=$ 
14.0, 8.4 Hz, 1H), 2.25-2.07 (m, 2H). $\left.{ }^{13} \mathrm{C} \mathrm{NMR} \mathrm{(100} \mathrm{MHz,} \mathrm{CDCl}_{3}\right) \delta$ 150.8, 137.2, 133.9, 133.5, 130.7, 128.7, 127.5, 126.3, 125.5, 124.5, 118.7, 108.4, 97.8, 67.4, 57.6, 41.6, 39.7. IR (thin film): $v_{\max }\left(\mathrm{cm}^{-1}\right)=3343,3024,2976,2948,2921,2893,2850$, 2146, 2103, 2087, 1938, 1883, 1857, 1736, 1648, 1601, 1483, 1462, 1429, 1382, 1362, 1347, 1333, 1285, 1226, 1198, 1157, 1119, 1061, 1030, 1012, 984, 962, 919, 893, 845, 794, 741, 693, 679, 619, 591, 527, 500, 470, 448. HRMS-ESI calcd for $\mathrm{C}_{19} \mathrm{H}_{19} \mathrm{CINO}[\mathrm{M}+\mathrm{H}]^{+}: 312.1150$. Found: 312.1154.

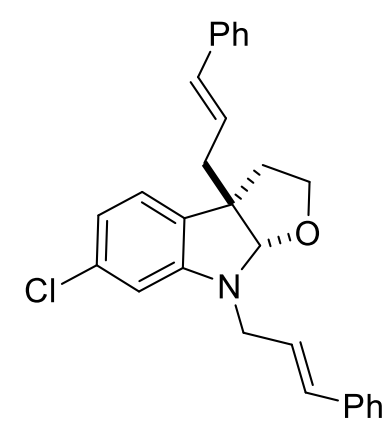

3e', yellow solid, m.p. $=105.6-106.2{ }^{\circ} \mathrm{C} .18 .9 \mathrm{mg}, 22 \%$ yield. ${ }^{1} \mathrm{H}$ NMR $(400 \mathrm{MHz}$, $\left.\mathrm{CDCl}_{3}\right) \delta 7.28-7.17(\mathrm{~m}, 10 \mathrm{H}), 6.97(\mathrm{~d}, J=7.6 \mathrm{~Hz}, 1 \mathrm{H}), 6.64(\mathrm{dd}, J=8.0,2.0 \mathrm{~Hz}, 1 \mathrm{H})$, $6.52(\mathrm{~d}, J=16.0 \mathrm{~Hz}, 1 \mathrm{H}), 6.44(\mathrm{~d}, J=16.0 \mathrm{~Hz}, 1 \mathrm{H}), 6.38(\mathrm{~d}, J=1.6 \mathrm{~Hz}, 1 \mathrm{H}), 6.12$ (dt, $J=15.6,5.2 \mathrm{~Hz}, 1 \mathrm{H}), 6.03(\mathrm{ddd}, J=15.2,8.8,6.0 \mathrm{~Hz}, 1 \mathrm{H}), 5.37(\mathrm{~s}, 1 \mathrm{H}), 4.06-3.96(\mathrm{~m}$, $3 \mathrm{H}), 3.51$ (ddd, $J=11.2,8.8,5.2 \mathrm{~Hz}, 1 \mathrm{H}), 2.78(\mathrm{ddd}, J=13.6,6.0,1.2 \mathrm{~Hz}, 1 \mathrm{H}), 2.59$ $(\mathrm{dd}, J=14.0,8.8 \mathrm{~Hz}, 1 \mathrm{H}), 2.23-2.06(\mathrm{~m}, 2 \mathrm{H}) .{ }^{13} \mathrm{C} \mathrm{NMR}\left(100 \mathrm{MHz}, \mathrm{CDCl}_{3}\right) \delta 151.5$, 137.2 , 136.7, 134.2, 133.5, 132.1, 131.1, 128.7, 128.6, 127.6, 127.5, 126.5, 126.3, 125.6, 125.1, 124.1, 117.2, 105.6, 101.4, 67.1, 56.2, 46.4, 41.9, 40.4. IR (thin film): $v_{\max }\left(\mathrm{cm}^{-1}\right)=3055,3025,2963,2924,2872,2388,1875,1658,1602,1584,1491$, 1447, 1418, 1360, 1327, 1298, 1251, 1206, 1181, 1156, 1127, 1116, 1070, 1044, 1010, 960, 939, 910, 892, 867, 818, 751, 731, 689, 609, 530, 506, 463, 420. HRMS-ESI calcd for $\mathrm{C}_{28} \mathrm{H}_{27} \mathrm{ClNO}[\mathrm{M}+\mathrm{H}]^{+}:$428.1776. Found: 428.1769 .<smiles>Cc1cccc2c1N[C@@H]1OCC[C@]21C/C=C/c1ccccc1</smiles> 
3f, yellow solid, m.p. $=104.0-105.4{ }^{\circ} \mathrm{C} .22 .2 \mathrm{mg}, 38 \%$ yield. ${ }^{1} \mathrm{H}$ NMR $(400 \mathrm{MHz}$, $\left.\mathrm{CDCl}_{3}\right) \delta 7.33-7.26(\mathrm{~m}, 4 \mathrm{H}), 7.23-7.17(\mathrm{~m}, 1 \mathrm{H}), 6.96(\mathrm{~d}, J=7.6 \mathrm{~Hz}, 1 \mathrm{H}), 6.92(\mathrm{~d}, J=$ $7.2 \mathrm{~Hz}, 1 \mathrm{H}), 6.71(\mathrm{t}, J=7.2 \mathrm{~Hz}, 1 \mathrm{H}), 6.45(\mathrm{~d}, J=15.6 \mathrm{~Hz}, 1 \mathrm{H}), 6.13(\mathrm{ddd}, J=15.2$, 8.4, $6.8 \mathrm{~Hz}, 1 \mathrm{H}), 5.44$ (s, 1H), 4.44 (br s, 1H), 3.97 (ddd, $J=8.4,7.2,1.6 \mathrm{~Hz}, 1 \mathrm{H}$ ), 3.57 (ddd, $J=10.8,8.4,5.2 \mathrm{~Hz}, 1 \mathrm{H}), 2.73$ (ddd, $J=14.0,6.4,1.2 \mathrm{~Hz}, 1 \mathrm{H}$ ), 2.64 (ddd, $J=14.0,8.4,0.8 \mathrm{~Hz}, 1 \mathrm{H}), 2.28-2.09(\mathrm{~m}, 2 \mathrm{H}), 2.15(\mathrm{~s}, 3 \mathrm{H}) .{ }^{13} \mathrm{C} \mathrm{NMR}(100 \mathrm{MHz}$, $\left.\mathrm{CDCl}_{3}\right) \delta 148.2,137.5,133.2,131.7,129.3,128.6,127.4,126.3,126.1,121.3,119.2$, 118.0, 97.6, 67.4 , 58.4, 41.8, 39.6, 16.9. IR (thin film): $v_{\max }\left(\mathrm{cm}^{-1}\right)=3393,3348,3186$, 3101, 3049, 2920, 2884, 2850, 2357, 2341, 2290, 2122, 1960, 1944, 1893, 1849, 1805, 1719, 1646, 1599, 1541, 1483, 1467, 1444, 1418, 1383, 1358, 1331, 1314, 1261, 1224, 1196, 1156, 1127, 1086, 1070, 1051, 1038, 1020, 957, 930, 898, 864, 844, 811, 772, 744, 691, 637, 608, 549, 500. HRMS-ESI calcd for $\mathrm{C}_{20} \mathrm{H}_{22} \mathrm{NO}[\mathrm{M}+\mathrm{H}]^{+}: 292.1696$. Found: 292.1696.

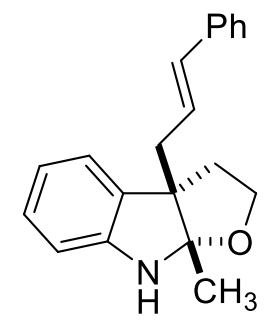

3g, yellow solid, m.p. $=102.6-103.3{ }^{\circ} \mathrm{C} .52 .0 \mathrm{mg}, 89 \%$ yield. ${ }^{1} \mathrm{H}$ NMR $(400 \mathrm{MHz}$, $\left.\mathrm{CDCl}_{3}\right) \delta$ 7.36-7.28 (m, 4H), 7.23-7.18 (m, 1H), 7.12-7.02 (m, 2H), 6.78-6.66 (m, $J=$ $6.6 \mathrm{~Hz}, 1 \mathrm{H}), 6.58(\mathrm{~d}, J=5.6 \mathrm{~Hz}, 1 \mathrm{H}), 6.41(\mathrm{~d}, J=16.0 \mathrm{~Hz}, 1 \mathrm{H}), 6.28-6.12(\mathrm{~m}, 1 \mathrm{H})$, 4.50 (br s, 1H), 3.97-3.82 (m, 1H), 3.52-3.41 (m, 1H), 2.62 (dd, $J=14.0,6.4 \mathrm{~Hz}, 1 \mathrm{H})$, $2.50(\mathrm{dd}, J=13.6,7.6 \mathrm{~Hz}, 1 \mathrm{H}), 2.30-2.23(\mathrm{~m}, 1 \mathrm{H}), 2.22-2.12(\mathrm{~m}, 1 \mathrm{H}), 1.54(\mathrm{~s}, 3 \mathrm{H})$. ${ }^{13} \mathrm{C} \mathrm{NMR}\left(100 \mathrm{MHz}, \mathrm{CDCl}_{3}\right) \delta 148.6,137.6,133.1,132.4,128.6,128.2,127.3,126.4$, 126.2, 124.4, 118.9, 108.7, 103.4, 66.4, 57.5, 39.7, 39.5, 22.6. IR (thin film): $v_{\max }\left(\mathrm{cm}^{-}\right.$ $\left.{ }^{1}\right)=3300,3049,3027,2975,2925,2873,2332,2120,1990,1965,1887,1768,1647$, $1608,1594,1485,1468,1448,1419,1375,1346,1329,1293,1263,1238,1219,1202$, 1133, 1109, 1083, 1063, 1031, 964, 928, 881, 843, 818, 798, 744, 697, 664, 629, 571, 544, 504, 467, 447. HRMS-ESI calcd for $\mathrm{C}_{20} \mathrm{H}_{22} \mathrm{NO}[\mathrm{M}+\mathrm{H}]^{+}$: 292.1696. Found: 292.1701. 


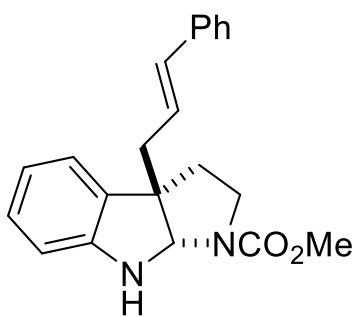

3h, yellow solid, m.p. $=116.3-117.1{ }^{\circ} \mathrm{C}$ (Known compound, see: Kumar, N.; Maity, A.; Gavit, V. R.; Bisai, A. Chem. Commun. 2018, 54, 9083). $63.5 \mathrm{mg}, 95 \%$ yield. ${ }^{1} \mathrm{H}$ NMR (400 MHz, $\mathrm{CDCl}_{3}$ ) (two sets of signals were observed due to the existence of rotamers) $\delta$ 7.37-7.25 (m, 4H), 7.23-7.17 (m, 1H), 7.12-7.04 (m, 2H), 6.80-6.71 (m, $1 \mathrm{H}), 6.60(\mathrm{dd}, J=7.6,4.4 \mathrm{~Hz}, 1 \mathrm{H}), 6.41(\mathrm{~d}, J=16.0 \mathrm{~Hz}, 1 \mathrm{H}), 6.14(\mathrm{ddd}, J=23.2$, 15.2, 7.2 Hz, 1H), 5.19, 5.13 (s, 1H), 3.75, 3.67 (s, 3H), 3.77-3.57 (m, 1H), 3.14-2.97 (m, 1H), 2.66-2.52 (m, 2H), 2.25-2.08 (m, 2H).

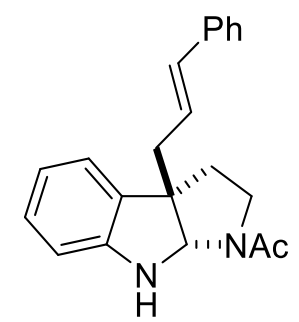

3i, yellow solid, m.p. $=154.1-155.0{ }^{\circ} \mathrm{C} .32 .9 \mathrm{mg}, 52 \%$ yield. ${ }^{1} \mathrm{H}$ NMR $(400 \mathrm{MHz}$, $\left.\mathrm{CDCl}_{3}\right) \delta$ 7.35-7.26 (m, 4H), 7.24-7.18 (m, 1H), 7.11-7.06 (m, 2H), $6.75(\mathrm{td}, J=7.6$, $1.2 \mathrm{~Hz}, 1 \mathrm{H}), 6.60(\mathrm{dd}, J=7.6,0.4 \mathrm{~Hz}, 1 \mathrm{H}), 6.42(\mathrm{~d}, J=16.0 \mathrm{~Hz}, 1 \mathrm{H}), 6.23-6.10$ (m, $1 \mathrm{H}), 5.29(\mathrm{~s}, 1 \mathrm{H}), 3.58(\mathrm{ddd}, J=10.0,7.6,2.0 \mathrm{~Hz}, 1 \mathrm{H}), 3.20(\mathrm{td}, J=10.4,6.8 \mathrm{~Hz}, 1 \mathrm{H})$, $2.59(\mathrm{dd}, J=7.6,0.8 \mathrm{~Hz}, 2 \mathrm{H}), 2.32-2.19(\mathrm{~m}, 2 \mathrm{H}), 1.99$ (s, 3H). ${ }^{13} \mathrm{C} \mathrm{NMR}(100 \mathrm{MHz}$, $\left.\mathrm{CDCl}_{3}\right) \delta 170.3,149.4,137.3,133.7,131.3,128.7,128.6,127.4,126.3,125.3,123.3$, 118.8, 109.5, 79.9, 56.4, 47.3, 41.1, 34.9, 22.8. IR (thin film): $v_{\max }\left(\mathrm{cm}^{-1}\right)=3323$, 3027, 2928, 2880, 2848, 2392, 2302, 1951, 1892, 1809, 1778, 1724, 1631, 1603, 1484, 1468, 1438, 1421, 1358, 1332, 1312, 1259, 1217, 1169, 1152, 1128, 1102, 1065, 1017, 964, 905, 879, 800, 750, 643, 623, 585, 513, 496, 462, 430. HRMS-ESI calcd for $\mathrm{C}_{21} \mathrm{H}_{23} \mathrm{~N}_{2} \mathrm{O}[\mathrm{M}+\mathrm{H}]^{+}:$319.1805. Found: 319.1805. 


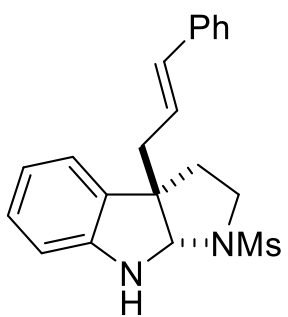

3j, yellow solid, m.p. $=124.2-125.8{ }^{\circ} \mathrm{C} .48 .6 \mathrm{mg}, 69 \%$ yield. ${ }^{1} \mathrm{H}$ NMR $(400 \mathrm{MHz}$, $\left.\mathrm{CDCl}_{3}\right) \delta$ 7.33-7.27 (m, 4H), 7.25-7.18 (m, 1H), 7.16-7.07 (m, 2H), $6.83(\mathrm{t}, J=7.2 \mathrm{~Hz}$, $1 \mathrm{H}), 6.66(\mathrm{~d}, J=7.6 \mathrm{~Hz}, 1 \mathrm{H}), 6.44(\mathrm{~d}, J=15.6 \mathrm{~Hz}, 1 \mathrm{H}), 6.09-5.97(\mathrm{~m}, 1 \mathrm{H}), 5.30(\mathrm{~s}$, $1 \mathrm{H}), 4.74$ (br s, 1H), 3.62-3.51 (m, 1H), 3.15-3.03 (m, 1H), 2.89 (s, 3H), 2.70-2.63 (m, $1 \mathrm{H}), 2.59(\mathrm{dd}, J=13.6,8.0 \mathrm{~Hz}, 1 \mathrm{H}), 2.31-2.24(\mathrm{~m}, 2 \mathrm{H}) .{ }^{13} \mathrm{C} \mathrm{NMR}\left(100 \mathrm{MHz}, \mathrm{CDCl}_{3}\right)$ $\delta 149.0,137.1,133.9,131.8,128.9,128.7,127.5,126.2,124.9,123.4,119.9,110.0$, 82.1, 58.8, 47.0, 42.1, 38.8, 36.6. IR (thin film): $v_{\max }\left(\mathrm{cm}^{-1}\right)=3364,3080,3056,3029$, 2920, 2892, 2848, 2105, 2087, 1921, 1886, 1646, 1608, 1483, 1464, 1436, 1410, 1361, 1304, 1241, 1202, 1151, 1105, 1083, 1050, 1036, 1022, 969, 911, 879, 859, 813, 765, 739, 691, 652, 619, 603, 550, 537, 513, 496, 466, 450. HRMS-ESI calcd for $\mathrm{C}_{20} \mathrm{H}_{23} \mathrm{~N}_{2} \mathrm{O}_{2} \mathrm{~S}[\mathrm{M}+\mathrm{H}]^{+}:$355.1475. Found: 355.1486.

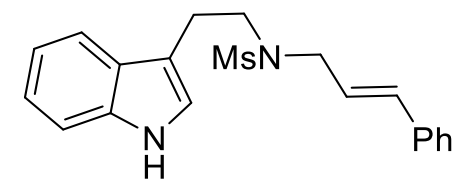

4j, yellow solid, m.p. $=101.6-102.0{ }^{\circ} \mathrm{C} .11 .1 \mathrm{mg}, 16 \%$ yield. ${ }^{1} \mathrm{H}$ NMR $(400 \mathrm{MHz}$, $\left.\mathrm{CDCl}_{3}\right) \delta 8.02(\mathrm{br} \mathrm{s}, 1 \mathrm{H}), 7.57(\mathrm{~d}, J=8.0 \mathrm{~Hz}, 1 \mathrm{H}), 7.38-7.30(\mathrm{~m}, 5 \mathrm{H}), 7.30-7.26(\mathrm{~m}$, 1H), $7.19(\mathrm{t}, J=7.6 \mathrm{~Hz}, 1 \mathrm{H}), 7.10-7.04(\mathrm{~m}, 2 \mathrm{H}), 6.53(\mathrm{~d}, J=16.0 \mathrm{~Hz}, 1 \mathrm{H}), 6.17(\mathrm{dt}, J$ $=16.0,7.2 \mathrm{~Hz}, 1 \mathrm{H}), 4.01(\mathrm{~d}, J=6.8 \mathrm{~Hz}, 2 \mathrm{H}), 3.59-3.50(\mathrm{~m}, 2 \mathrm{H}), 3.15-3.06(\mathrm{~m}, 2 \mathrm{H})$, $2.79(\mathrm{~s}, 3 \mathrm{H}) .{ }^{13} \mathrm{C} \mathrm{NMR}\left(100 \mathrm{MHz}, \mathrm{CDCl}_{3}\right) \delta 136.3,136.2,134.5,128.8,128.3,127.3$, $126.7,124.0,122.4,122.3,119.7,118.8,112.6,111.4,50.3,47.8,39.3,25.4$. IR (thin film): $v_{\max }\left(\mathrm{cm}^{-1}\right)=3414,3024,2956,2921,2850,2387,2112,1875,1645,1552$, 1493, 1452, 1425, 1320, 1209, 1139, 1116, 1079, 1044, 1009, 955, 918, 854, 826, 797, 749, 729, 693, 640, 598, 537, 516, 497, 462, 443, 422. HRMS-ESI calcd for $\mathrm{C}_{20} \mathrm{H}_{23} \mathrm{~N}_{2} \mathrm{O}_{2} \mathrm{~S}[\mathrm{M}+\mathrm{H}]^{+}:$355.1475. Found: 355.1476. 


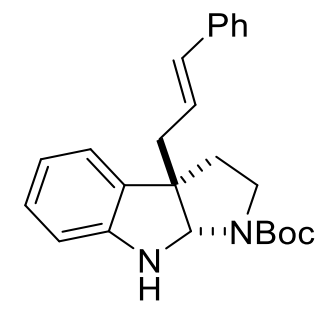

3k, yellow oil (Known compound, see: Kumar, N.; Maity, A.; Gavit, V. R.; Bisai, A. Chem. Commun. 2018, 54, 9083). $70.6 \mathrm{mg}, 94 \%$ yield. ${ }^{1} \mathrm{H}$ NMR (400 MHz, $\mathrm{CDCl}_{3}$ ) (two sets of signals were observed due to the existence of rotamers) $\delta 7.34-7.21(\mathrm{~m}$, 4H), 7.20-7.13 (m, 1H), 7.09-7.01 (m, 2H), $6.73(\mathrm{q}, J=7.6 \mathrm{~Hz}, 1 \mathrm{H}), 6.58(\mathrm{t}, J=8.4$ $\mathrm{Hz}, 1 \mathrm{H}), 6.38(\mathrm{~d}, J=15.6 \mathrm{~Hz}, 1 \mathrm{H}), 6.21-6.08(\mathrm{~m}, 1 \mathrm{H}), 5.16,5.06(\mathrm{~s}, 1 \mathrm{H}), 4.70(\mathrm{br} \mathrm{s}$, 1H), 3.73-3.62, 3.60-3.49 (m, 1H), 3.07-2.94 (m, 1H), 2.55 (t, $J=6.8$ Hz, 2H), 2.19$2.03(\mathrm{~m}, 2 \mathrm{H}), 1.51,1.43(\mathrm{~s}, 9 \mathrm{H})$.

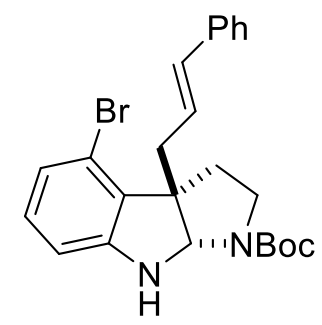

31, yellow solid, m.p. $=88.4-89.9{ }^{\circ} \mathrm{C} .70 .6 \mathrm{mg}, 78 \%$ yield. ${ }^{1} \mathrm{H}$ NMR $(400 \mathrm{MHz}$, $\mathrm{CDCl}_{3}$ ) (two sets of signals were observed due to the existence of rotamers) $\delta 7.25$ (d, $J=4.4 \mathrm{~Hz}, 4 \mathrm{H}), 7.21-7.14(\mathrm{~m}, 1 \mathrm{H}), 6.92-6.81(\mathrm{~m}, 2 \mathrm{H}), 6.48(\mathrm{~d}, J=7.6 \mathrm{~Hz}, 1 \mathrm{H}), 6.44$ $(\mathrm{d}, J=16.0 \mathrm{~Hz}, 1 \mathrm{H}), 6.01(\mathrm{ddd}, J=22.8,15.2,7.6 \mathrm{~Hz}, 1 \mathrm{H}), 5.24,5.13$ (s, 1H), 3.70, $3.56(\mathrm{t}, J=9.2 \mathrm{~Hz}, 1 \mathrm{H}), 3.14-2.95(\mathrm{~m}, 2 \mathrm{H}), 2.86(\mathrm{dd}, J=12.8,6.0 \mathrm{~Hz}, 1 \mathrm{H}), 2.71-2.61$ $(\mathrm{m}, 1 \mathrm{H}), 2.12-1.98(\mathrm{~m}, 1 \mathrm{H}), 1.51(\mathrm{~s}, 3 \mathrm{H}), 1.44(\mathrm{~s}, 6 \mathrm{H}) .{ }^{13} \mathrm{C} \mathrm{NMR}\left(100 \mathrm{MHz}, \mathrm{CDCl}_{3}\right)$ (two sets of signals were observed due to the existence of rotamers) $\delta 154.6,153.5$, $151.6,151.3,137.4,133.4,133.2,130.1,128.5,128.4,128.2,127.2,126.3,125.4$, 125.3, 123.2, 122.8, 119.1, 119.0, 108.1, 108.0, 80.4, 80.0, 79.2, 60.0, 58.9, 45.7, 45.3, 39.1, 38.8, 32.9, 32.8, 28.8, 28.6. IR (thin film): $v_{\max }\left(\mathrm{cm}^{-1}\right)=3370,3080,3022,2980$, 2924, 2881, 2116, 2080, 1896, 1803, 1670, 1598, 1570, 1495, 1476, 1453, 1402, 1363, 1304, 1251, 1219, 1166, 1113, 1083, 1048, 960, 915, 888, 859, 809, 765, 731, 691, 639, 619, 585, 558, 530, 502. HRMS-ESI calcd for $\mathrm{C}_{24} \mathrm{H}_{28}{ }^{79} \mathrm{BrN}_{2} \mathrm{O}_{2}[\mathrm{M}+\mathrm{H}]^{+}$: 455.1329. Found: 455.1330. 
<smiles>COc1ccc2c(c1)[C@]1(C/C=C/c3ccccc3)CC[C@H](N2)[C@H]1C(C)(C)C</smiles>

3m, yellow oil (Known compound, see: Liu, Y.; Du, H. Org. Lett. 2013, 15, 740). $63.4 \mathrm{mg}, 78 \%$ yield. ${ }^{1} \mathrm{H}$ NMR (400 MHz, $\mathrm{CDCl}_{3}$ ) (two sets of signals were observed due to the existence of rotamers) $\delta 7.42-7.24(\mathrm{~m}, 4 \mathrm{H}), 7.23-7.15(\mathrm{~m}, J=6.3 \mathrm{~Hz}, 1 \mathrm{H})$, 6.72-6.67 (m, 1H), 6.65 (dd, $J=8.4,2.4 \mathrm{~Hz}, 1 \mathrm{H}), 6.55$ (d, $J=8.4 \mathrm{~Hz}, 1 \mathrm{H}), 6.42$ (d, $J$ $=16.0 \mathrm{~Hz}, 1 \mathrm{H}), 6.25-6.09(\mathrm{~m}, 1 \mathrm{H}), 5.16,5.06(\mathrm{~s}, 1 \mathrm{H}), 3.71(\mathrm{~s}, 3 \mathrm{H}), 3.75-3.65,3.60-$ $3.51(\mathrm{~m}, 1 \mathrm{H}), 3.10-2.97(\mathrm{~m}, 1 \mathrm{H}), 2.58(\mathrm{t}, J=6.4 \mathrm{~Hz}, 2 \mathrm{H}), 2.21-2.08(\mathrm{~m}, 2 \mathrm{H}), 1.51(\mathrm{~s}$, $4 \mathrm{H}), 1.44(\mathrm{~s}, 5 \mathrm{H})$.

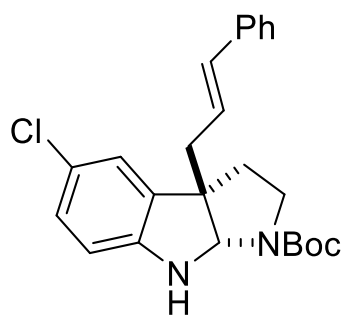

3n, yellow oil. $72.1 \mathrm{mg}, 88 \%$ yield. ${ }^{1} \mathrm{H}$ NMR (400 MHz, $\mathrm{CDCl}_{3}$ ) (two sets of signals were observed due to the existence of rotamers) $\delta 7.38-7.25(\mathrm{~m}, 4 \mathrm{H}), 7.23-7.18(\mathrm{~m}$, 1H), 7.06-6.99 (m, 2H), 6.55-6.49 (m, 1H), 6.42 (d, $J=15.6 \mathrm{~Hz}, 1 \mathrm{H}), 6.17-6.04(\mathrm{~m}$, 1H), 5.18, 5.08 (s, 1H), 5.13, 4.65 (br s, 1H), 3.76-3.63, 3.62-3.50 (m, 1H), 3.11-2.96 $(\mathrm{m}, 1 \mathrm{H}), 2.64-2.49(\mathrm{~m}, 2 \mathrm{H}), 2.21-2.09(\mathrm{~m}, 2 \mathrm{H}), 1.51(\mathrm{~s}, 3 \mathrm{H}), 1.44(\mathrm{~s}, 6 \mathrm{H}) .{ }^{13} \mathrm{C}$ NMR $\left(100 \mathrm{MHz}, \mathrm{CDCl}_{3}\right.$ ) (two sets of signals were observed due to the existence of rotamers) $\delta 154.6,153.4,148.1,147.8,137.2,134.0,133.9,133.8,128.64,128.61$, $128.4,128.3,127.5,127.4,126.3,124.9,123.6,123.5,123.1,110.2,110.1,80.5,80.2$, 80.1, 58.1, 57.0, 45.8, 45.4, 41.4, 41.1, 34.85, 34.82, 28.8, 28.5. IR (thin film): $v_{\max }\left(\mathrm{cm}^{-1}\right)=3422,3027,2977,2931,2875,2138,1948,1874,1675,1603,1479,1450$, 1428, 1393, 1366, 1320, 1278, 1254, 1208, 1160, 1114, 1039, 966, 907, 879, 827, 810, 773, 736, 691, 646, 625, 584, 524, 459. HRMS-ESI calcd for $\mathrm{C}_{24} \mathrm{H}_{28} \mathrm{ClN}_{2} \mathrm{O}_{2}[\mathrm{M}+\mathrm{H}]^{+}$: 411.1834. Found: 411.1830. 


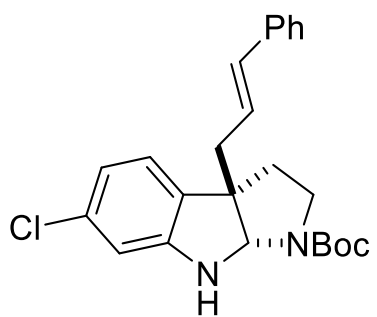

3o, colorless oil. $59.8 \mathrm{mg}, 73 \%$ yield. ${ }^{1} \mathrm{H}$ NMR (400 $\mathrm{MHz}, \mathrm{CDCl}_{3}$ ) (two sets of signals were observed due to the existence of rotamers) $\delta 7.35-7.25(\mathrm{~m}, 4 \mathrm{H}), 7.24$ $7.17(\mathrm{~m}, 1 \mathrm{H}), 6.93,6.88$ (d, $J=7.6 \mathrm{~Hz}$; d, $J=8.0 \mathrm{~Hz} ; 1 \mathrm{H}), 6.86-6.80,6.57$ (m; dd, $J=$ 9.2, $1.6 \mathrm{~Hz} ; 1 \mathrm{H}), 6.76-6.64(\mathrm{~m}, 1 \mathrm{H}), 6.39(\mathrm{~d}, J=15.6 \mathrm{~Hz}, 1 \mathrm{H}), 6.20-6.03(\mathrm{~m}, 1 \mathrm{H})$, $5.22,4.75$ (br s, 1H), 5.16, $5.06(\mathrm{~d}, J=5.6 \mathrm{~Hz}, 1 \mathrm{H}), 3.73-3.63,3.60-3.50(\mathrm{~m}, 1 \mathrm{H})$, 3.07-2.95 (m, 1H), 2.61-2.46 (m, 2H), 2.21-2.05 (m, 2H), 1.51, $1.44(\mathrm{~s}, 9 \mathrm{H}) .{ }^{13} \mathrm{C}$ NMR (100 MHz, $\mathrm{CDCl}_{3}$ ) (two sets of signals were observed due to the existence of rotamers) $\delta 154.5,153.4,150.9,150.6,150.5,150.3,137.2,134.0,133.9,133.72$, 133.69, 133.67, 133.6, 131.0, 130.8, 130.4, 130.2, 128.61, 128.58, 127.43, 127.38, 126.2 , 125.05, 125.01, 124.96, 124.7, 124.5, 124.2, 124.1, 122.02, 121.99, 121.6, $121.2,118.8,118.3,112.3,112.2,109.45,109.38,80.4,80.2,80.1,80.0,57.5,57.4$, $56.4,56.3,45.8,45.4,41.5,41.4,41.25,41.17,34.9,34.8,34.7,28.7,28.5$. IR (thin film): $v_{\max }\left(\mathrm{cm}^{-1}\right)=3352,3028,2970,2929,2914,2882,2836,2135,2102,1942$, 1893, 1678, 1604, 1508, 1482, 1398, 1365, 1302, 1263, 1240, 1161, 1111, 1028, 962 , $910,886,840,798,774,740,693,658,621,573,559,520,470,449$. HRMS-ESI calcd for $\mathrm{C}_{24} \mathrm{H}_{28} \mathrm{ClN}_{2} \mathrm{O}_{2}[\mathrm{M}+\mathrm{H}]^{+}:$411.1834. Found: 411.1832 .

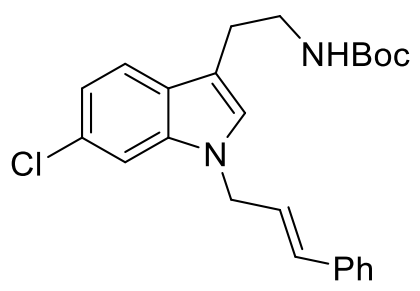

3o', colorless oil. $16.4 \mathrm{mg}, 20 \%$ yield. ${ }^{1} \mathrm{H}$ NMR (400 MHz, $\left.\mathrm{CDCl}_{3}\right) \delta 7.50$ (d, $J=8.4$ $\mathrm{Hz}, 1 \mathrm{H}), 7.37-7.27$ (m, 5H), 7.27-7.21 (m, 1H), 7.08 (dd, J = 8.4, 2.0 Hz, 1H), 6.97 (s, $1 \mathrm{H}), 6.48(\mathrm{~d}, J=15.6 \mathrm{~Hz}, 1 \mathrm{H}), 6.30(\mathrm{dt}, J=15.6,5.6 \mathrm{~Hz}, 1 \mathrm{H}), 4.79(\mathrm{dd}, J=6.0,1.2$ $\mathrm{Hz}, 2 \mathrm{H}), 4.60(\mathrm{~s}, 1 \mathrm{H}), 3.43(\mathrm{~d}, J=6.0 \mathrm{~Hz}, 2 \mathrm{H}), 2.91(\mathrm{t}, J=6.8 \mathrm{~Hz}, 2 \mathrm{H}), 1.42(\mathrm{~s}, 9 \mathrm{H})$. ${ }^{13} \mathrm{C} \mathrm{NMR}\left(100 \mathrm{MHz}, \mathrm{CDCl}_{3}\right) \delta 156.0,137.0,136.1,132.8,128.8,128.2,128.0,126.8$, $126.7,126.4,124.4,120.1,119.9,112.7,109.7,79.3,48.4,41.1,28.5,25.8$. IR (thin film): $v_{\max }\left(\mathrm{cm}^{-1}\right)=3431,3355,2973,2921,2852,2384,1694,1610,1502,1467$, 
1391, 1364, 1324, 1245, 1164, 1066, 1025, 964, 909, 859, 837, 801, 732, 693, 663, 647, 599, 577, 530, 496, 465, 426. HRMS-ESI calcd for $\mathrm{C}_{24} \mathrm{H}_{28} \mathrm{ClN}_{2} \mathrm{O}_{2}[\mathrm{M}+\mathrm{H}]^{+}$: 411.1834. Found: 411.1832.

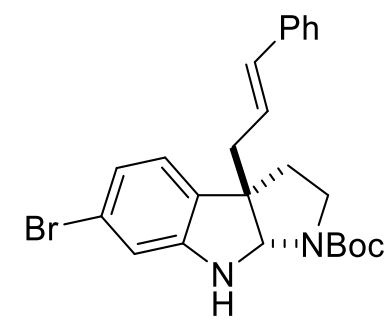

3p, yellow solid, m.p. $=66.0-66.8{ }^{\circ} \mathrm{C} .71 .9 \mathrm{mg}, 79 \%$ yield. ${ }^{1} \mathrm{H}$ NMR $(400 \mathrm{MHz}$, $\mathrm{CDCl}_{3}$ ) (two sets of signals were observed due to the existence of rotamers) $\delta 7.34$ $7.26(\mathrm{~m}, 4 \mathrm{H}), 7.24-7.18(\mathrm{~m}, 1 \mathrm{H}), 6.90(\mathrm{~d}, J=7.6 \mathrm{~Hz}, 1 \mathrm{H}), 6.88-6.82(\mathrm{~m}, 1 \mathrm{H}), 6.77-$ $6.72(\mathrm{~m}, 1 \mathrm{H}), 6.40(\mathrm{~d}, J=15.6 \mathrm{~Hz}, 1 \mathrm{H}), 6.18-6.04(\mathrm{~m}, 1 \mathrm{H}), 5.15,5.05$ (s, 1H), 3.743.65, 3.61-3.52 (m, 1H), 3.07-2.96 (m, 1H), 2.64-2.48 (m, 2H), 2.21-2.07 (m, 2H), $1.51(\mathrm{~s}, 3 \mathrm{H}), 1.44(\mathrm{~s}, 6 \mathrm{H}),{ }^{13} \mathrm{C} \mathrm{NMR}\left(100 \mathrm{MHz} \mathrm{CDCl}_{3}\right)$ (two sets of signals were observed due to the existence of rotamers) $\delta 154.5,153.4,150.9,150.5,137.2,133.8$, $133.7,131.0,130.8,128.7,128.6,127.5,127.4,126.3,125.05,125.02,124.7,124.6$, $122.1,122.0,121.7,121.3,112.4,112.3,80.5,80.20,80.17,80.1,57.5,56.5,45.9$, 45.5, 41.4, 41.2, 34.9, 34.8, 28.8, 28.6. IR (thin film): $v_{\max }\left(\mathrm{cm}^{-1}\right)=3364,3079,3056$, 3028, 2930, 2891, 2849, 2357, 2309, 1952, 1920, 1882, 1803, 1677, 1606, 1481, 1464, 1436, 1405, 1363, 1304, 1240, 1202, 1152, 1109, 1083, 1049, 1036, 968, 910, $880,859,812,765,739,691,651,618,602,550,536,513,466,450$. HRMS-ESI calcd for $\mathrm{C}_{24} \mathrm{H}_{28}{ }^{79} \mathrm{BrN}_{2} \mathrm{O}_{2}[\mathrm{M}+\mathrm{H}]^{+}: 455.1329$. Found: 455.1331 .

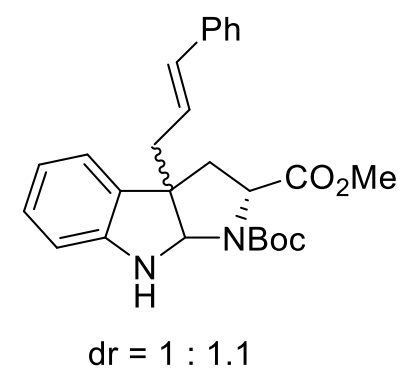

3q(major), yellow oil. $42.6 \mathrm{mg}, 49 \%$ yield. $[\alpha]_{\mathrm{D}}{ }^{27}=-180.8\left(\mathrm{c}=0.6, \mathrm{CHCl}_{3}\right) .{ }^{1} \mathrm{H} \mathrm{NMR}$ (400 $\mathrm{MHz}, \mathrm{CDCl}_{3}$ ) (two sets of signals were observed due to the existence of rotamers) $\delta$ 7.36-7.26 (m, 4H), 7.25-7.18 (m, 1H), 7.10-7.01 (m, 1H), 7.01-6.94 (m, 
$1 \mathrm{H}), 6.73-6.59(\mathrm{~m}, 2 \mathrm{H}), 6.42(\mathrm{~d}, J=15.6 \mathrm{~Hz}, 1 \mathrm{H}), 6.23-6.09(\mathrm{~m}, 1 \mathrm{H}), 5.23,5.12(\mathrm{~s}$, 1H), 4.76 (br s, 1H), 4.58, 4.43 (d, $J=8.4 \mathrm{~Hz}$; d, $J=7.6 \mathrm{~Hz} ; 1 \mathrm{H}), 3.16,3.15$ (s, 3H), 2.67-2.42 (m, 4H), $1.55(\mathrm{~s}, 3 \mathrm{H}), 1.40(\mathrm{~s}, 6 \mathrm{H}) .{ }^{13} \mathrm{C} \mathrm{NMR}\left(100 \mathrm{MHz}, \mathrm{CDCl}_{3}\right.$ ) (two sets of signals were observed due to the existence of rotamers) $\delta 172.2,171.7,154.2$, 153.5, 150.1, 149.8, 137.31, 137.29, 133.8, 133.7, 130.9, 130.6, 129.0, 128.9, 128.7, $128.6,127.5,127.4,126.31,126.29,125.1$, 124.0, 123.7, 119.0, 118.6, 109.5, 109.4, 81.1, 81.0, 80.8, 80.6, 59.7, 59.3, 57.2, 56.1, 52.1, 52.0, 41.3, 41.0, 39.0, 38.8, 28.7, 28.4. IR (thin film): $v_{\max }\left(\mathrm{cm}^{-1}\right)=3396,3054,2974,2925,2849,2390,1755,1732$, 1687, 1608, 1483, 1451, 1433, 1388, 1323, 1275, 1256, 1225, 1201, 1166, 1112, 1095, 1057, 1019, 967, 905, 858, 811, 741, 693, 664, 613, 535, 498, 468. HRMS-ESI calcd for $\mathrm{C}_{26} \mathrm{H}_{31} \mathrm{~N}_{2} \mathrm{O}_{4}[\mathrm{M}+\mathrm{H}]^{+}$: 435.2278. Found: 435.2276.

3q(minor), yellow solid, m.p. $=106.0-106.9 .39 .1 \mathrm{mg}, 45 \%$ yield. $[\alpha]_{\mathrm{D}}{ }^{27}=251.5(\mathrm{c}=$ $0.5, \mathrm{CHCl}_{3}$ ). ${ }^{1} \mathrm{H} \mathrm{NMR}\left(400 \mathrm{MHz}, \mathrm{CDCl}_{3}\right.$ ) (two sets of signals were observed due to the existence of rotamers) $\delta 7.36-7.26(\mathrm{~m}, 4 \mathrm{H}), 7.24-7.18(\mathrm{~m}, 1 \mathrm{H}), 7.13-7.05(\mathrm{~m}, 2 \mathrm{H})$, 6.80-6.74 (m, 1H), $6.64(\mathrm{dd}, J=12.0,8.0 \mathrm{~Hz}, 1 \mathrm{H}), 6.41(\mathrm{dd}, J=15.6,4.4 \mathrm{~Hz}, 1 \mathrm{H})$, 6.19-6.05 (m, 1H), 5.40, $4.87(\mathrm{~s}, 1 \mathrm{H}), 5.33,5.24(\mathrm{~s}, 1 \mathrm{H}), 4.10-4.00(\mathrm{~m}, 1 \mathrm{H}), 3.73,3.70$ $(\mathrm{s}, 3 \mathrm{H}), 2.66-2.52(\mathrm{~m}, 3 \mathrm{H}), 2.28-2.19(\mathrm{~m}, 1 \mathrm{H}), 1.52,1.37(\mathrm{~s}, 9 \mathrm{H}) .{ }^{13} \mathrm{C}$ NMR $(100$ $\mathrm{MHz}, \mathrm{CDCl}_{3}$ ) (two sets of signals were observed due to the existence of rotamers) $\delta$ $173.6,173.1,153.9,153.5,148.8,148.4,137.3,133.8,133.7,131.7,131.5,128.82$, 128.76, 128.65, 128.63, 127.5, 127.4, 126.3, 125.1, 125.0, 123.5, 119.3, 119.0, 109.9, 109.6, 81.41, 81.35, 81.0, 80.9, 59.50, 59.47, 57.0, 56.0, 52.4, 52.2, 41.1, 41.0, 39.5, 39.3, 28.7, 28.3. IR (thin film): $v_{\max }\left(\mathrm{cm}^{-1}\right)=3427,3003,2971,2921,2850,1742$, $1686,1649,1608,1479,1463,1396,1362,1335,1304,1273,1256,1228,1201$, 1172, 1157, 1128, 1097, 1047, 1010, 969, 920, 901, 848, 777, 741, 696, 656, 613, 580, 531, 499, 469. HRMS-ESI calcd for $\mathrm{C}_{26} \mathrm{H}_{31} \mathrm{~N}_{2} \mathrm{O}_{4}[\mathrm{M}+\mathrm{H}]^{+}$: 435.2278. Found: 435.2273 . 


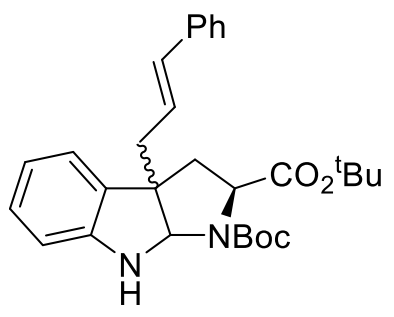

$\mathrm{dr}=1: 1.3$

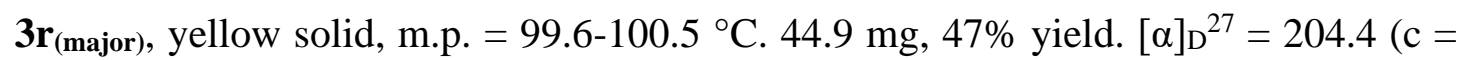
0.45, $\mathrm{CHCl}_{3}$ ). ${ }^{1} \mathrm{H} \mathrm{NMR}\left(400 \mathrm{MHz}, \mathrm{CDCl}_{3}\right.$ ) (two sets of signals were observed due to the existence of rotamers) $\delta 7.36-7.26(\mathrm{~m}, 4 \mathrm{H}), 7.24-7.18(\mathrm{~m}, 1 \mathrm{H}), 7.09-7.02(\mathrm{~m}, 1 \mathrm{H})$, $7.00(\mathrm{~d}, J=7.6 \mathrm{~Hz}, 1 \mathrm{H}), 6.72-6.65(\mathrm{~m}, 1 \mathrm{H}), 6.62(\mathrm{dd}, J=14.8,7.2 \mathrm{~Hz}, 1 \mathrm{H}), 6.41(\mathrm{~d}, J$ $=16.0 \mathrm{~Hz}, 1 \mathrm{H}), 6.20-6.07(\mathrm{~m}, 1 \mathrm{H}), 5.23,5.12(\mathrm{~s}, 1 \mathrm{H}), 5.19,4.77$ (br s, 1H), 4.46, 4.36-4.28 (d, $J=8.8 \mathrm{~Hz} ; \mathrm{m} ; 1 \mathrm{H}), 2.63-2.43(\mathrm{~m}, 4 \mathrm{H}), 1.54$ (s, 2H), 1.43 (s, 7H), 1.08, $1.07(\mathrm{~s}, 9 \mathrm{H}) .{ }^{13} \mathrm{C} \mathrm{NMR}\left(100 \mathrm{MHz}, \mathrm{CDCl}_{3}\right.$ ) (two sets of signals were observed due to the existence of rotamers) $\delta 170.8,170.2,154.2,153.3,149.9,149.6,137.4,133.8$, $133.7,131.5,131.2$, 128.9, 128.8, 128.7, 128.6, 127.4, 126.32, 126.30, 125.2, 124.0, 123.7, 119.1, 118.7, 109.38, 109.36, 81.09, 81.06, 80.82, 80.79, 80.7, 80.4, 60.3, 60.0, $57.2,56.1,42.1,41.8,39.3,38.9,28.7,28.5,27.54,27.52$. IR (thin film): $v_{\max }\left(\mathrm{cm}^{-1}\right)=$ 3319, 3054, 3031, 2974, 2928, 2451, 2380, 2348, 2323, 2297, 1713, 1682, 1606, $1484,1471,1450,1395,1365,1325,1283,1256,1224,1171,1129,1110,1059,1040$, $967,916,883,847,815,764,739,694,675,631,610,586,533,500$, 447. HRMSESI calcd for $\mathrm{C}_{29} \mathrm{H}_{37} \mathrm{~N}_{2} \mathrm{O}_{4}[\mathrm{M}+\mathrm{H}]^{+}:$477.2748. Found: 477.2743.

3r(minor), yellow oil. $40.8 \mathrm{mg}, 43 \%$ yield. $[\alpha]_{\mathrm{D}}{ }^{26}=-218.6\left(\mathrm{c}=1.0, \mathrm{CHCl}_{3}\right) .{ }^{1} \mathrm{H}$ NMR $\left(400 \mathrm{MHz}, \mathrm{CDCl}_{3}\right.$ ) (two sets of signals were observed due to the existence of rotamers) $\delta$ 7.35-7.25 (m, 4H), 7.23-7.17 (m, 1H), 7.13-7.05 (m, 2H), 6.80-6.72 (m, 1H), 6.67-6.58 (m, 1H), 6.41 (dd, $J=15.6,4.8 \mathrm{~Hz}, 1 \mathrm{H}), 6.17-6.05(\mathrm{~m}, 1 \mathrm{H}), 5.34,5.22$ (s, 1H), 4.04-3.96 (m, 1H), 2.66-2.53 (m, 3H), 2.25-2.15 (m, 1H), 1.52 (s, 3H), 1.45 (s, 9H), $1.41(\mathrm{~s}, 6 \mathrm{H}) \cdot{ }^{13} \mathrm{C} \mathrm{NMR}\left(100 \mathrm{MHz}, \mathrm{CDCl}_{3}\right)$ (two sets of signals were observed due to the existence of rotamers) $\delta 172.3,171.8,154.5,153.5,148.7,148.4,137.34$, $137.31,133.8,133.6,132.4,132.0,128.7,128.61,128.59$, 127.41, 127.39, 126.3, $125.31,125.26,123.5,123.4,119.2,118.8,109.8,109.6,81.7,81.3,81.03,80.95$, $80.8,60.5,60.0,56.9,55.7,41.2,41.0,39.8,39.3,28.7,28.4,28.1,28.0$. IR (thin film): $v_{\max }\left(\mathrm{cm}^{-1}\right)=3395,3055,3004,2975,2923,2850,1738,1690,1608,1482$, 
1466, 1390, 1366, 1314, 1257, 1213, 1151, 1095, 1045, 964, 911, 847, 787, 741, 693, 665, 617, 586, 546, 501, 478. HRMS-ESI calcd for $\mathrm{C}_{29} \mathrm{H}_{37} \mathrm{~N}_{2} \mathrm{O}_{4}[\mathrm{M}+\mathrm{H}]^{+}: 477.2748$. Found: 477.2743.

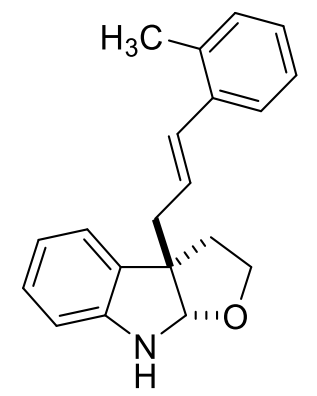

3s, brown oil. $43.0 \mathrm{mg}, 74 \%$ yield. ${ }^{1} \mathrm{H} \mathrm{NMR}\left(400 \mathrm{MHz}, \mathrm{CDCl}_{3}\right) \delta$ 7.35-7.26 (m, 1H), 7.15-7.08 (m, 4H), $7.05(\mathrm{t}, J=7.6 \mathrm{~Hz}, 1 \mathrm{H}), 6.74(\mathrm{t}, J=7.6 \mathrm{~Hz}, 1 \mathrm{H}), 6.64-6.54(\mathrm{~m}$, 2H), 6.01-5.91 (m, 1H), $5.42(\mathrm{~s}, 1 \mathrm{H}), 4.61$ (br s, 1H), $3.97(\mathrm{t}, J=8.0 \mathrm{~Hz}, 1 \mathrm{H}), 3.57$ (ddd, $J=10.8,8.8,5.6 \mathrm{~Hz}, 1 \mathrm{H}), 2.77(\mathrm{dd}, J=14.0,6.8 \mathrm{~Hz}, 1 \mathrm{H}), 2.64(\mathrm{dd}, J=13.6$, $8.0 \mathrm{~Hz}, 1 \mathrm{H}), 2.26$ (s, 3H), 2.24-2.12 (m, 2H). $\left.{ }^{13} \mathrm{C} \mathrm{NMR} \mathrm{(100} \mathrm{MHz,} \mathrm{CDCl}_{3}\right) \delta$ 149.7, $136.7,135.2$, 132.2, 131.3, 130.2, 128.3, 127.4, 127.3, 126.1, 125.8, 123.8, 118.9, 108.4, 97.7, 67.3, 58.1, 42.0, 39.7, 19.9. IR (thin film): $v_{\max }\left(\mathrm{cm}^{-1}\right)=3355,3183$, 3051, 3019, 2918, 2851, 2347, 2113, 2088, 2026, 1997, 1915, 1890, 1720, 1658, $1631,1608,1483,1467,1427,1380,1359,1333,1315,1255,1219,1165,1153,1113$, 1033, 1011, 963, 915, 815, 740, 659, 621, 577, 535, 506, 448. HRMS-ESI calcd for $\mathrm{C}_{20} \mathrm{H}_{22} \mathrm{NO}[\mathrm{M}+\mathrm{H}]^{+}:$292.1696. Found: 292.1695.

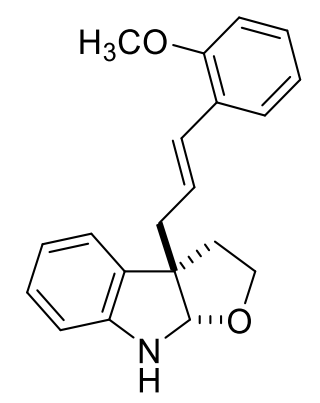

3t, yellow oil. $55.2 \mathrm{mg}, 90 \%$ yield. ${ }^{1} \mathrm{H} \mathrm{NMR}\left(400 \mathrm{MHz}, \mathrm{CDCl}_{3}\right) \delta 7.32(\mathrm{dd}, J=7.6$, $1.2 \mathrm{~Hz}, 1 \mathrm{H}), 7.18(\mathrm{td}, J=8.0,1.2 \mathrm{~Hz}, 1 \mathrm{H}), 7.13-7.10(\mathrm{~m}, 1 \mathrm{H}), 7.06(\mathrm{td}, J=7.6,1.2 \mathrm{~Hz}$, $1 \mathrm{H}), 6.88(\mathrm{t}, J=7.2 \mathrm{~Hz}, 1 \mathrm{H}), 6.84(\mathrm{~d}, J=8.4 \mathrm{~Hz}, 1 \mathrm{H}), 6.80-6.73(\mathrm{~m}, 2 \mathrm{H}), 6.58(\mathrm{~d}, J=$ $8.0 \mathrm{~Hz}, 1 \mathrm{H}), 6.09$ (ddd, $J=15.2,8.4,6.4 \mathrm{~Hz}, 1 \mathrm{H}), 5.42$ (s, 1H), 4.60 (br s, 1H), 4.00$3.92(\mathrm{~m}, 1 \mathrm{H}), 3.81(\mathrm{~s}, 3 \mathrm{H}), 3.57$ (ddd, $J=11.2,8.8,5.2 \mathrm{~Hz}, 1 \mathrm{H}), 2.76(\mathrm{ddd}, J=14.0$, 
6.4, $1.2 \mathrm{~Hz}, 1 \mathrm{H}), 2.69-2.61(\mathrm{~m}, 1 \mathrm{H}), 2.28-2.11(\mathrm{~m}, 2 \mathrm{H}) .{ }^{13} \mathrm{C} \mathrm{NMR}\left(100 \mathrm{MHz}, \mathrm{CDCl}_{3}\right)$ $\delta 156.5,149.7,132.4,128.3,128.2,127.9,126.7,126.6,126.5,123.8,120.7,118.9$, 110.9, 108.4, 97.6, 67.3, 58.1, 55.5, 42.0, 39.6. IR (thin film): $v_{\max }\left(\mathrm{cm}^{-1}\right)=3393$, $3354,3045,2922,2865,2835,2260,2111,1985,1930,1682,1647,1607,1598$, 1580, 1485, 1464, 1435, 1358, 1334, 1315, 1292, 1241, 1175, 1161, 1108, 1049, $1025,970,917,852,744,659,617,578,542,460$. HRMS-ESI calcd for $\mathrm{C}_{20} \mathrm{H}_{22} \mathrm{NO}_{2}$ $[\mathrm{M}+\mathrm{H}]^{+}:$308.1645. Found: 308.1643.

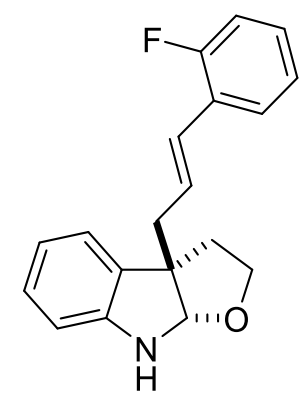

3u, yellow solid, m.p. $=65.5-66.7{ }^{\circ} \mathrm{C} .32 .9 \mathrm{mg}, 56 \%$ yield. ${ }^{1} \mathrm{H}$ NMR $(400 \mathrm{MHz}$, $\left.\mathrm{CDCl}_{3}\right) \delta 7.33(\mathrm{td}, J=7.6,0.8 \mathrm{~Hz}, 1 \mathrm{H}), 7.21-7.13(\mathrm{~m}, 1 \mathrm{H}), 7.13-6.95(\mathrm{~m}, 4 \mathrm{H}), 6.76(\mathrm{t}$, $J=7.2 \mathrm{~Hz}, 1 \mathrm{H}), 6.63-6.56(\mathrm{~m}, 2 \mathrm{H}), 6.18(\mathrm{ddd}, J=15.2,8.0,6.8 \mathrm{~Hz}, 1 \mathrm{H}), 5.41(\mathrm{~s}, 1 \mathrm{H})$, 4.61 (br s, 1H), 4.01-3.93 (m, 1H), 3.57 (ddd, $J=10.8,8.8,5.6 \mathrm{~Hz}, 1 \mathrm{H}), 2.77$ (dd, $J=$ 14.0, $6.8 \mathrm{~Hz}, 1 \mathrm{H}), 2.66$ (dd, $J=14.0,8.4 \mathrm{~Hz}, 1 \mathrm{H}), 2.27-2.11(\mathrm{~m}, 2 \mathrm{H}) .{ }^{19} \mathrm{~F}$ NMR $(376$ $\left.\mathrm{MHz}, \mathrm{CDCl}_{3}\right) \delta-118.5(\mathrm{~m}) .{ }^{13} \mathrm{C} \mathrm{NMR}\left(100 \mathrm{MHz}, \mathrm{CDCl}_{3}\right) \delta 160.1(\mathrm{~d}, J=248.7 \mathrm{~Hz})$, 149.7, 132.1, 128.8 (d, $J=4.6 \mathrm{~Hz}), 128.6(\mathrm{~d}, J=8.3 \mathrm{~Hz}), 128.4,127.3(\mathrm{~d}, J=3.9 \mathrm{~Hz})$, $125.6(\mathrm{~d}, J=3.5 \mathrm{~Hz}), 125.2(\mathrm{~d}, J=12.3 \mathrm{~Hz}), 124.1(\mathrm{~d}, J=3.5 \mathrm{~Hz}), 123.8,119.0$, $115.8(\mathrm{~d}, J=22.1 \mathrm{~Hz}), 108.5,97.6,67.4,58.0,42.0$, 39.6. IR (thin film): $v_{\max }\left(\mathrm{cm}^{-1}\right)=$ $3351,3187,3037,3015,2960,2920,2871,2851,2368,2357,2342,2307,2125$, 1991, 1949, 1917, 1881, 1839, 1801, 1765, 1713, 1647, 1608, 1577, 1483, 1471, 1453, 1363, 1339, 1317, 1294, 1254, 1227, 1186, 1168, 1151, 1113, 1096, 1051, 1030, 1009, 957, 910, 843, 794, 740, 691, 657, 607, 568, 533, 517, 461, 448. HRMS-ESI calcd for $\mathrm{C}_{19} \mathrm{H}_{19} \mathrm{FNO}[\mathrm{M}+\mathrm{H}]^{+}:$296.1445. Found: 296.1444 . 


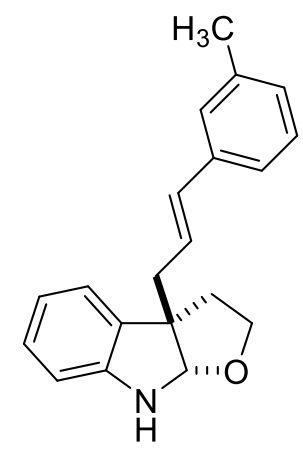

3v, yellow oil. $44.0 \mathrm{mg}, 76 \%$ yield. ${ }^{1} \mathrm{H}$ NMR (400 MHz, $\left.\mathrm{CDCl}_{3}\right) \delta 7.17(\mathrm{t}, J=7.2 \mathrm{~Hz}$, 1H), 7.14-7.05 (m, 4H), $7.03(\mathrm{t}, J=6.8 \mathrm{~Hz}, 1 \mathrm{H}), 6.75(\mathrm{t}, J=7.6 \mathrm{~Hz}, 1 \mathrm{H}), 6.57$ (d, $J=$ $7.6 \mathrm{~Hz}, 1 \mathrm{H}), 6.41(\mathrm{~d}, J=16.0 \mathrm{~Hz}, 1 \mathrm{H}), 6.10$ (ddd, $J=14.8,8.4,6.8 \mathrm{~Hz}, 1 \mathrm{H}), 5.40$ (s, 1H), 4.62 (br s, 1H), 4.03-3.89 (m, 1H), 3.56 (ddd, $J=10.8,8.8,5.2 \mathrm{~Hz}, 1 \mathrm{H}), 2.73(\mathrm{dd}$, $J=14.0,6.4 \mathrm{~Hz}, 1 \mathrm{H}), 2.62(\mathrm{dd}, J=13.6,8.0 \mathrm{~Hz}, 1 \mathrm{H}), 2.32(\mathrm{~s}, 3 \mathrm{H}), 2.26-2.17(\mathrm{~m}, 1 \mathrm{H})$, $2.14(\mathrm{dd}, J=11.6,4.8 \mathrm{~Hz}, 1 \mathrm{H}) .{ }^{13} \mathrm{C} \mathrm{NMR}\left(100 \mathrm{MHz}, \mathrm{CDCl}_{3}\right) \delta 149.6,138.2,137.3$, 133.4, 132.2, 128.5, 128.3, 128.1, 127.0, 125.7, 123.8, 123.4, 118.9, 108.4, 97.6, 67.3, 58.0, 41.7, 39.6, 21.5. IR (thin film): $v_{\max }\left(\mathrm{cm}^{-1}\right)=3343,3024,2921,2865,2732$, 2675, 2244, 1932, 1881, 1607, 1484, 1467, 1444, 1360, 1333, 1314, 1254, 1219, 1167, 1153, 1113, 1035, 1012, 963, 908, 777, 740, 696, 661, 622, 576, 505, 438. HRMS-ESI calcd for $\mathrm{C}_{20} \mathrm{H}_{22} \mathrm{NO}[\mathrm{M}+\mathrm{H}]^{+}:$292.1696. Found: 292.1696.

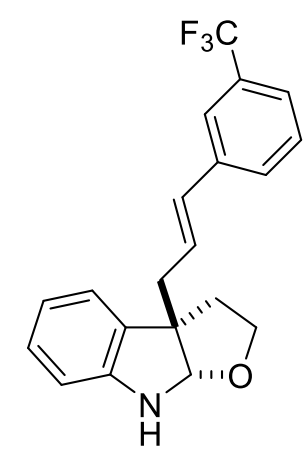

3w, yellow oil. $44.8 \mathrm{mg}, 65 \%$ yield. ${ }^{1} \mathrm{H}$ NMR $\left(400 \mathrm{MHz}, \mathrm{CDCl}_{3}\right) \delta 7.51(\mathrm{~s}, 1 \mathrm{H}), 7.45$ $(\mathrm{d}, J=8.0 \mathrm{~Hz}, 2 \mathrm{H}), 7.41-7.35(\mathrm{~m}, 1 \mathrm{H}), 7.12-7.05(\mathrm{~m}, 2 \mathrm{H}), 6.77(\mathrm{td}, J=7.2,0.4 \mathrm{~Hz}$, $1 \mathrm{H}), 6.60(\mathrm{~d}, J=7.6 \mathrm{~Hz}, 1 \mathrm{H}), 6.46(\mathrm{~d}, J=15.6 \mathrm{~Hz}, 1 \mathrm{H}), 6.18$ (ddd, $J=14.8,8.0,6.4$ $\mathrm{Hz}, 1 \mathrm{H}), 5.41$ (s, 1H), 4.60 (br s, 1H), 3.98 (ddd, $J=8.4,6.8,1.6 \mathrm{~Hz}, 1 \mathrm{H}), 3.58$ (ddd, $J=10.4,8.8,5.6 \mathrm{~Hz}, 1 \mathrm{H}), 2.78(\mathrm{ddd}, J=14.0,6.4,1.2 \mathrm{~Hz}, 1 \mathrm{H}), 2.66(\mathrm{dd}, J=14.0$, $8.0 \mathrm{~Hz}, 1 \mathrm{H}), 2.27-2.14(\mathrm{~m}, 2 \mathrm{H}) .{ }^{19} \mathrm{~F} \mathrm{NMR}\left(376 \mathrm{MHz}, \mathrm{CDCl}_{3}\right) \delta-62.7(\mathrm{~m}) .{ }^{13} \mathrm{C} \mathrm{NMR}$ $\left(100 \mathrm{MHz} \mathrm{CDCl}_{3}\right) \delta 149.7,138.1,132.0,131.9,131.0(\mathrm{q}, J=31.9 \mathrm{~Hz}), 129.3(\mathrm{~d}, J=$ $1.1 \mathrm{~Hz}), 129.1,128.5,128.1,124.2$ (d, $J=272.3 \mathrm{~Hz}), 123.9$ (q, $J=3.7 \mathrm{~Hz}), 123.7$, 
$123.0(\mathrm{q}, J=3.8 \mathrm{~Hz}), 119.0,108.5,97.6,67.4,58.0,41.6,39.6$. IR (thin film): $v_{\max }\left(\mathrm{cm}^{-1}\right)=3349,3032,2940,2870,2120,1886,1652,1609,1484,1468,1442,1326$, 1258, 1220, 1201, 1162, 1119, 1096, 1071, 1035, 1012, 963, 903, 877, 794, 743, 697, $662,613,578,536,512,451$. HRMS-ESI calcd for $\mathrm{C}_{20} \mathrm{H}_{19} \mathrm{~F}_{3} \mathrm{NO}[\mathrm{M}+\mathrm{H}]^{+}: 346.1413$. Found: 346.1411.

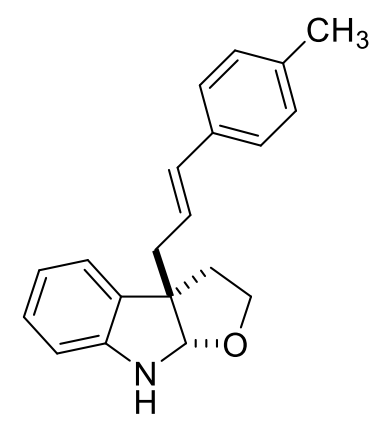

3x, yellow solid, m.p. $=62.3-63.1{ }^{\circ} \mathrm{C} .47 .4 \mathrm{mg}, 81 \%$ yield. ${ }^{1} \mathrm{H}$ NMR $(400 \mathrm{MHz}$, $\left.\mathrm{CDCl}_{3}\right) \delta 7.19(\mathrm{~d}, J=8.0 \mathrm{~Hz}, 2 \mathrm{H}), 7.12-7.03(\mathrm{~m}, 4 \mathrm{H}), 6.75(\mathrm{td}, J=7.6,0.8 \mathrm{~Hz}, 1 \mathrm{H})$, $6.58(\mathrm{~d}, J=7.6 \mathrm{~Hz}, 1 \mathrm{H}), 6.41(\mathrm{~d}, J=15.6 \mathrm{~Hz}, 1 \mathrm{H}), 6.06(\mathrm{ddd}, J=15.2,8.4,6.4 \mathrm{~Hz}$, $1 \mathrm{H}), 5.40$ (s, 1H), 4.58 (br s, 1H), 4.00-3.92 (m, 1H), 3.56 (ddd, $J=10.8,8.8,5.2 \mathrm{~Hz}$, 1H), $2.73(\mathrm{ddd}, J=13.6,6.4,1.2 \mathrm{~Hz}, 1 \mathrm{H}), 2.61$ (dd, $J=14.0,8.4 \mathrm{~Hz}, 1 \mathrm{H}), 2.32$ (s, $3 \mathrm{H}), 2.26-2.11(\mathrm{~m}, 2 \mathrm{H}) .{ }^{13} \mathrm{C} \mathrm{NMR}\left(100 \mathrm{MHz}, \mathrm{CDCl}_{3}\right) \delta 149.7,137.1,134.6,133.1$, 132.3, 129.3, 128.3, 126.2, 124.9, 123.8, 118.9, 108.5, 97.7, 67.4, 58.1, 41.7, 39.6, 21.3. IR (thin film): $v_{\max }\left(\mathrm{cm}^{-1}\right)=3345,3083,3023,2920,2865,2830,2730,2682$, 2294, 2110, 2090, 1901, 1774, 1713, 1676, 1647, 1608, 1511, 1483, 1468, 1448, 1426, 1360, 1336, 1313, 1262, 1221, 1154, 1111, 1073, 1037, 1000, 975, 942, 895, 877, 829, 802, 773, 743, 696, 661, 617, 575, 520, 499, 453, 414. HRMS-ESI calcd for $\mathrm{C}_{20} \mathrm{H}_{22} \mathrm{NO}[\mathrm{M}+\mathrm{H}]^{+}:$292.1696. Found: 292.1695.

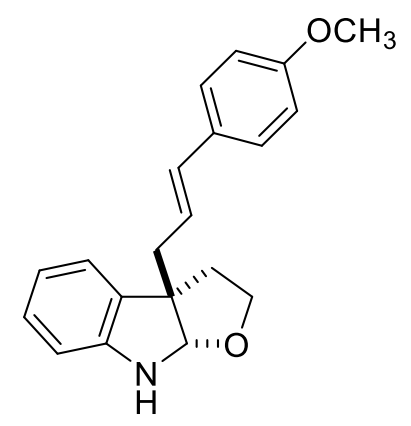


3y, yellow solid, m.p. $=116.2-117.0{ }^{\circ} \mathrm{C} .49 .4 \mathrm{mg}, 80 \%$ yield. ${ }^{1} \mathrm{H}$ NMR $(400 \mathrm{MHz}$, $\left.\mathrm{CDCl}_{3}\right) \delta 7.23(\mathrm{~d}, J=8.8 \mathrm{~Hz}, 2 \mathrm{H}), 7.12-7.04(\mathrm{~m}, 2 \mathrm{H}), 6.82(\mathrm{~d}, J=8.8 \mathrm{~Hz}, 2 \mathrm{H}), 6.75$ $(\mathrm{td}, J=7.6,0.8 \mathrm{~Hz}, 1 \mathrm{H}), 6.59(\mathrm{~d}, J=7.6 \mathrm{~Hz}, 1 \mathrm{H}), 6.39$ (d, $J=15.6 \mathrm{~Hz}, 1 \mathrm{H}), 6.02-5.92$ (m, 1H), 5.40 (s, 1H), 4.59 (br s, 1H), 4.03-3.91 (m, 1H), 3.79 (s, 3H), 3.57 (ddd, $J=$ 10.8, 8.8, 5.2 Hz, 1H), $2.72(\mathrm{ddd}, J=14.0,6.4,1.2 \mathrm{~Hz}, 1 \mathrm{H}), 2.61(\mathrm{dd}, J=14.0,8.4 \mathrm{~Hz}$, $1 \mathrm{H}), 2.26-2.11(\mathrm{~m}, 2 \mathrm{H}) .{ }^{13} \mathrm{C} \mathrm{NMR}\left(100 \mathrm{MHz}, \mathrm{CDCl}_{3}\right) \delta$ 159.0, 149.7, 132.6, 132.3, 130.2 , 128.2, 127.4, 123.8, 123.7, 118.9, 114.0, 108.4, 97.6, 67.3, 58.1, 55.4, 41.7, 39.6. IR (thin film): $v_{\max }\left(\mathrm{cm}^{-1}\right)=3354,3031,2992,2968,2916,2836,2391,2306$, 2109, 1894, 1774, 1708, 1648, 1606, 1578, 1509, 1484, 1469, 1418, 1364, 1338, 1317 , 1298, 1264, 1241, 1177, 1153, 1133, 1111, 1064, 1028, 961, 942, 913, 840, 799, 782, $741,662,624,576,523,478,449$. HRMS-ESI calcd for $\mathrm{C}_{20} \mathrm{H}_{22} \mathrm{NO}_{2}[\mathrm{M}+\mathrm{H}]^{+}$: 308.1645 Found: 308.1643.

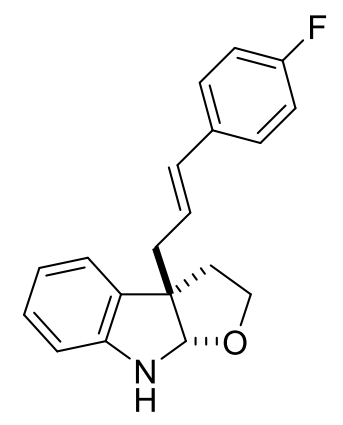

3z, yellow solid, m.p. $=57.3-58.2{ }^{\circ} \mathrm{C} .48 .7 \mathrm{mg}, 82 \%$ yield. ${ }^{1} \mathrm{H}$ NMR $(400 \mathrm{MHz}$, $\left.\mathrm{CDCl}_{3}\right) \delta$ 7.28-7.20 (m, 2H), 7.12-7.03 (m, 2H), 6.99-6.92 (m, 2H), $6.76(\mathrm{td}, J=7.6$, $0.4 \mathrm{~Hz}, 1 \mathrm{H}), 6.59$ (d, $J=7.6 \mathrm{~Hz}, 1 \mathrm{H}), 6.40$ (d, $J=16.0 \mathrm{~Hz}, 1 \mathrm{H}), 6.01$ (ddd, $J=15.2$, 8.0, $6.8 \mathrm{~Hz}, 1 \mathrm{H}), 5.40$ (s, 1H), 4.59 (br s, 1H), 4.02-3.90 (m, 1H), 3.57 (ddd, $J=10.8$, 8.8, 5.6 Hz, 1H), 2.79-2.68 (m, 1H), $2.62(\mathrm{dd}, J=14.0,8.4 \mathrm{~Hz}, 1 \mathrm{H}), 2.27-2.07$ (m, 2H). ${ }^{19} \mathrm{~F} \mathrm{NMR}\left(376 \mathrm{MHz}, \mathrm{CDCl}_{3}\right) \delta-115.0(\mathrm{~m}) .{ }^{13} \mathrm{C} \mathrm{NMR}\left(100 \mathrm{MHz}, \mathrm{CDCl}_{3}\right) \delta 162.2$ $(\mathrm{d}, J=246.2 \mathrm{~Hz}), 149.7,133.5(\mathrm{~d}, J=3.3 \mathrm{~Hz}), 132.1,132.0,128.3,127.7$ (d, $J=7.9$ $\mathrm{Hz}), 125.7(\mathrm{~d}, J=2.2 \mathrm{~Hz}), 123.7,118.9,115.4(\mathrm{~d}, J=21.5 \mathrm{~Hz}), 108.4,97.6,67.3$, 58.0, 41.6, 39.6. IR (thin film): $v_{\max }\left(\mathrm{cm}^{-1}\right)=3397,3052,2948,2920,2869,2851$, 2112, 1985, 1949, 1920, 1894, 1764, 1648, 1605, 1507, 1483, 1467, 1415, 1360, 1333, 1311, 1261, 1222, 1157, 1115, 1094, 1062, 1035, 1006, 966, 946, 920, 880, 842, 817, 786, 740, 694, 655, 620, 582, 498, 444. HRMS-ESI calcd for $\mathrm{C}_{19} \mathrm{H}_{19} \mathrm{FNO}[\mathrm{M}+\mathrm{H}]^{+}$: 296.1445. Found: 296.1444. 


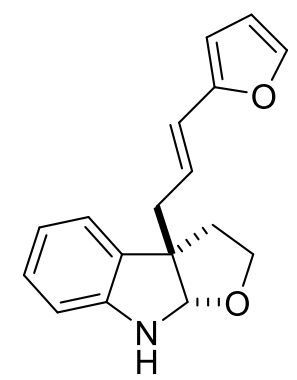

3aa, yellow oil. $34.8 \mathrm{mg}, 65 \%$ yield. ${ }^{1} \mathrm{H}$ NMR (400 MHz, $\left.\mathrm{CDCl}_{3}\right) \delta$ 7.31-7.28 (m, 1H), 7.11-7.03 (m, 2H), $6.75(\mathrm{t}, J=7.6 \mathrm{~Hz}, 1 \mathrm{H}), 6.58(\mathrm{~d}, J=8.0 \mathrm{~Hz}, 1 \mathrm{H}), 6.36-6.31$ (m, 1H), 6.25 (d, $J=15.6 \mathrm{~Hz}, 1 \mathrm{H}), 6.13$ (d, $J=3.2 \mathrm{~Hz}, 1 \mathrm{H}), 6.07$ (ddd, $J=15.2,8.0$, $6.8 \mathrm{~Hz}, 1 \mathrm{H}), 5.39$ (s, 1H), 4.61 (br s, 1H), 4.02-3.90 (m, 1H), 3.56 (ddd, $J=10.8,8.4$, $5.6 \mathrm{~Hz}, 1 \mathrm{H}), 2.75-2.65(\mathrm{~m}, 1 \mathrm{H}), 2.60(\mathrm{dd}, J=14.4,8.4 \mathrm{~Hz}, 1 \mathrm{H}), 2.25-2.10(\mathrm{~m}, 2 \mathrm{H})$. ${ }^{13} \mathrm{C} \mathrm{NMR}\left(100 \mathrm{MHz}, \mathrm{CDCl}_{3}\right) \delta 152.8,149.6,141.7,132.2,128.3,124.8,123.8,121.7$, 119.0, 111.2, 108.5, 107.0, 97.6, 67.3, 58.0, 41.5, 39.5. IR (thin film): $v_{\max }\left(\mathrm{cm}^{-1}\right)=$ 3345, 3146, 3116, 3049, 3032, 2938, 2868, 2236, 2115, 2082, 1984, 1921, 1891, 1718, 1658, 1607, 1562, 1484, 1467, 1445, 1360, 1334, 1314, 1254, 1222, 1206, 1150, 1114, 1033, 1010, 958, 922, 883, 797, 733, 659, 623, 593, 531, 508, 468, 450. HRMS-ESI calcd for $\mathrm{C}_{17} \mathrm{H}_{18} \mathrm{NO}_{2}[\mathrm{M}+\mathrm{H}]^{+}:$268.1332. Found: 268.1331 .

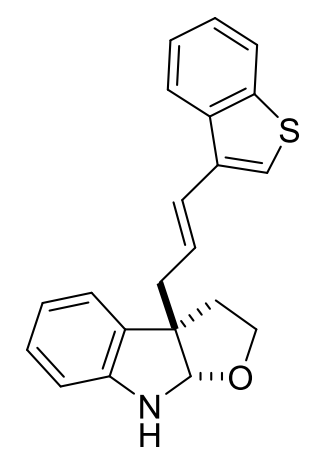

3ab, yellow solid, m.p. $=101.4-102.1{ }^{\circ} \mathrm{C} .52 .8 \mathrm{mg}, 79 \%$ yield. ${ }^{1} \mathrm{H}$ NMR $(400 \mathrm{MHz}$, $\left.\mathrm{CDCl}_{3}\right) \delta$ 7.85-7.79 (m, 1H), 7.77-7.71 (m, 1H), 7.38-7.30 (m, 2H), $7.27(\mathrm{~s}, 1 \mathrm{H}), 7.12$ $(\mathrm{d}, J=7.2 \mathrm{~Hz}, 1 \mathrm{H}), 7.08(\mathrm{td}, J=7.6,0.8 \mathrm{~Hz}, 1 \mathrm{H}), 6.80-6.73(\mathrm{~m}, 1 \mathrm{H}), 6.67$ (d, $J=16.0$ $\mathrm{Hz}, 1 \mathrm{H}), 6.59$ (d, $J=8.0 \mathrm{~Hz}, 1 \mathrm{H}), 6.13(\mathrm{ddd}, J=15.2,8.0,6.4 \mathrm{~Hz}, 1 \mathrm{H}), 5.45$ (s, 1H), 4.62 (br s, 1H), 4.06-3.92 (m, 1H), 3.59 (ddd, $J=10.4,8.4,5.6 \mathrm{~Hz}, 1 \mathrm{H}), 2.87-2.76$ (m, $1 \mathrm{H}), 2.67(\mathrm{dd}, J=13.6,8.0 \mathrm{~Hz}, 1 \mathrm{H}), 2.31-2.13(\mathrm{~m}, 2 \mathrm{H}) .{ }^{13} \mathrm{C} \mathrm{NMR}\left(100 \mathrm{MHz}, \mathrm{CDCl}_{3}\right)$ $\delta 149.8,140.4,137.8,134.2,132.0,128.4,128.2,125.5,124.4,124.2,123.8,122.9$, 122.0, 121.5, 119.0, 108.4, 97.6, 67.3, 58.0, 42.0, 39.8. IR (thin film): $v_{\max }\left(\mathrm{cm}^{-1}\right)=$ 3334, 3093, 3068, 3032, 2944, 2922, 2889, 2868, 2832, 2384, 2303, 2116, 1952, 1922 , 
1888, 1829, 1789, 1702, 1609, 1513, 1484, 1471, 1426, 1358, 1334, 1319, 1256, 1223, 1167, 1153, 1100, 1084, 1049, 1032, 1005, 955, 907, 870, 829, 797, 782, 748, 730, 697, 662, 627, 571, 528, 498, 448, 422. HRMS-ESI calcd for $\mathrm{C}_{21} \mathrm{H}_{20} \mathrm{NOS}[\mathrm{M}+\mathrm{H}]^{+}$: 334.1260. Found: 334.1260. 


\section{Transformation of products}

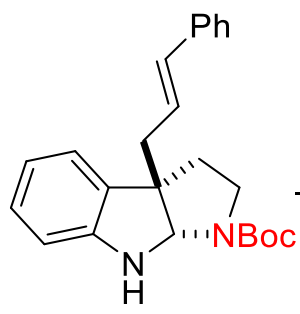

3I

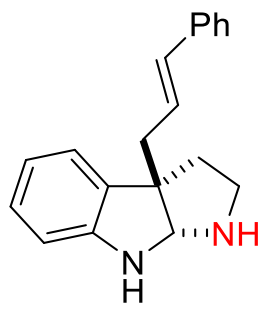

5

Trimethylsilyl trifluoromethanesulfonate ( $145 \mathrm{ul}, 0.8 \mathrm{mmol}, 4$ equiv) was added to a solution of 31 (75.3 mg, $0.2 \mathrm{mmol}, 1$ equiv) and 2,6-lutidine (116 ul, $1.0 \mathrm{mmol}, 5$ equiv) in DCM $(2 \mathrm{~mL})$ at $0{ }^{\circ} \mathrm{C}$. The reaction mixture was stirred at $0{ }^{\circ} \mathrm{C}$ for $30 \mathrm{~min}$. Then, the solution was stirred at room temperature. After the reaction was complete (monitored by TLC), the reaction was quenched by saturated sodium carbonate solution $(20 \mathrm{~mL})$. The mixture was extracted with DCM $(3 \times 15 \mathrm{~mL})$. The organic layer was washed with brine, dried over $\mathrm{Na}_{2} \mathrm{SO}_{4}$, filtered and evaporated under reduced pressure. Then the residue was purified by silica gel column chromatography $(\mathrm{EtOAc} / \mathrm{MeOH}=10 / 1)$ to afford 5.

5, white solid, m.p. $=89.1-89.9^{\circ} \mathrm{C} .45 .6 \mathrm{mg}, 82 \%$ yield. ${ }^{1} \mathrm{H} \mathrm{NMR}\left(400 \mathrm{MHz}, \mathrm{CDCl}_{3}\right)$ $\delta 8.42$ (br s, 1H), 7.42-7.31 (m, 4H), 7.31-7.24 (m, 1H), 7.22-7.10 (m, 2H), 6.88 (t, $J$ $=7.2 \mathrm{~Hz}, 1 \mathrm{H}), 6.75(\mathrm{~d}, J=7.6 \mathrm{~Hz}, 1 \mathrm{H}), 6.53(\mathrm{~d}, J=16.0 \mathrm{~Hz}, 1 \mathrm{H}), 6.22-6.09(\mathrm{~m}, 1 \mathrm{H})$, $5.50(\mathrm{~s}, 1 \mathrm{H}), 3.50-3.36(\mathrm{~m}, 1 \mathrm{H}), 2.99-2.81(\mathrm{~m}, 2 \mathrm{H}), 2.76(\mathrm{dd}, J=13.2,8.4 \mathrm{~Hz}, 1 \mathrm{H})$, 2.47-2.34 (m, 1H), $2.26(\mathrm{dd}, J=12.0,4.0 \mathrm{~Hz}, 1 \mathrm{H}) .{ }^{13} \mathrm{C} \mathrm{NMR}\left(100 \mathrm{MHz}, \mathrm{CDCl}_{3}\right) \delta$ 148.7 , 137.0, 134.2, 130.7, 129.1, 128.6, 127.5, 126.4, 124.6, 123.3, 120.0, 109.7, 82.0, 57.8, 44.0, 41.7, 37.6. IR (thin film): $v_{\max }\left(\mathrm{cm}^{-1}\right)=3369,3293,3024,2966,2917$, 2726, 2280, 2119, 1646, 1602, 1483, 1468, 1447, 1364, 1341, 1319, 1250, 1223, 1153, 1113, 1052, 1012, 969, 923, 904, 858, 743, 692, 637, 606, 557, 494, 457, 437. HRMS-ESI calcd for $\mathrm{C}_{19} \mathrm{H}_{21} \mathrm{~N}_{2}[\mathrm{M}+\mathrm{H}]^{+}:$277.1699. Found: 277.1704 . 


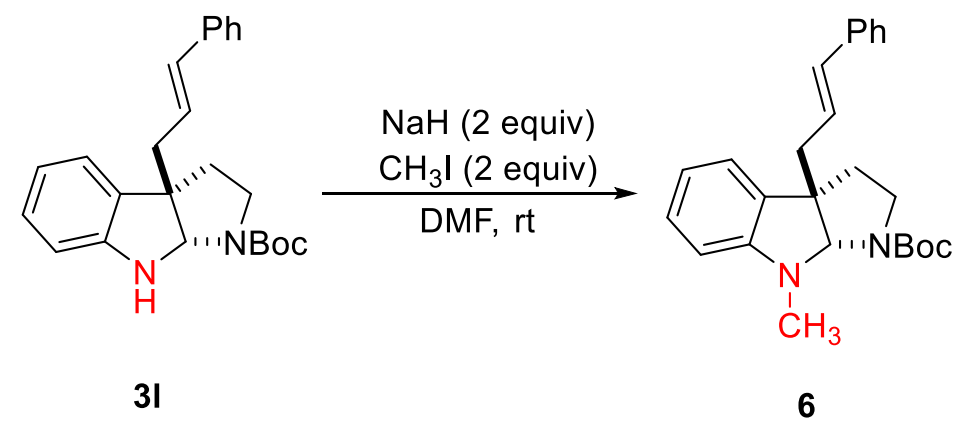

To a solution of $\mathbf{3 l}(37.7 \mathrm{mg}, 0.1 \mathrm{mmol}, 1$ equiv) in anhydrous $\mathrm{N}, \mathrm{N}-$ dimethylformamide $(1 \mathrm{~mL})$ was added sodium hydride (60 percent suspension in oil, $8.0 \mathrm{mg}, 0.2 \mathrm{mmol}, 2$ equiv) at $0{ }^{\circ} \mathrm{C}$. The reaction was stirred at $0{ }^{\circ} \mathrm{C}$ for $10 \mathrm{~min}$. Iodomethane (13 ul, $0.2 \mathrm{mmol}, 2$ equiv) was added and the reaction mixture was stirred at room temperature. After the reaction was complete (monitored by TLC), saturated ammonium chloride aqueous solution $(20 \mathrm{~mL})$ was added to quench the reaction. This reaction mixture was extracted with EtOAc $(3 \times 15 \mathrm{~mL})$. The extracts were combined, dried over $\mathrm{Na}_{2} \mathrm{SO}_{4}$, and evaporated under reduced pressure. The residue was purified by silica gel column chromatography $(\mathrm{PE} / \mathrm{EtOAc}=15 / 1)$ to afford the desired product 6.

6, colorless oil. $35.6 \mathrm{mg}, 91 \%$ yield. ${ }^{1} \mathrm{H} \mathrm{NMR}\left(400 \mathrm{MHz}, \mathrm{CDCl}_{3}\right.$ ) (two sets of signals were observed due to the existence of rotamers) $\delta 7.33-7.25(\mathrm{~m}, 4 \mathrm{H}), 7.21$ $7.16(\mathrm{~m}, 1 \mathrm{H}), 7.11(\mathrm{t}, J=8.0 \mathrm{~Hz}, 1 \mathrm{H}), 7.02(\mathrm{~d}, J=7.2 \mathrm{~Hz}, 1 \mathrm{H}), 6.67$ (t, $J=7.2 \mathrm{~Hz}$, $1 \mathrm{H}), 6.44(\mathrm{~d}, J=15.6 \mathrm{~Hz}, 1 \mathrm{H}), 6.38(\mathrm{~d}, J=7.6 \mathrm{~Hz}, 1 \mathrm{H}), 6.14-6.01(\mathrm{~m}, 1 \mathrm{H}), 5.33,5.18$ (s, 1H), 3.94-3.79, 3.78-3.61 (m, 1H), 3.11-2.98 (m, 1H), 2.96, 2.90 (s, 3H), 2.67 (dd, $J=14.0,6.8 \mathrm{~Hz}, 1 \mathrm{H}), 2.59(\mathrm{dd}, J=13.6,8.4 \mathrm{~Hz}, 1 \mathrm{H}), 2.11-2.02(\mathrm{~m}, 2 \mathrm{H}), 1.49,1.45$ (s, 9H). ${ }^{13} \mathrm{C}$ NMR $\left(100 \mathrm{MHz}, \mathrm{CDCl}_{3}\right.$ ) (two sets of signals were observed due to the existence of rotamers) $\delta 155.1,154.3,151.3,151.0,137.4,133.3,132.6,132.4,128.6$, $127.4,127.3,126.2,125.8,122.80,122.76,117.5,117.3,106.0,86.0,80.3,79.7,57.2$, 56.1, 46.3, 45.6, 42.0, 37.2, 36.8, 33.1, 32.7, 28.6, 28.5. IR (thin film): $v_{\max }\left(\mathrm{cm}^{-1}\right)=$ 3080, 3054, 3026, 3003, 2972, 2927, 2884, 2830, 2386, 2246, 1879, 1691, 1605, 1491, 1449, 1428, 1389, 1366, 1299, 1242, 1224, 1153, 1097, 1069, 1021, 987, 959, 923, 884, 736, 692, 646, 607, 549, 498, 473. HRMS-ESI calcd for $\mathrm{C}_{25} \mathrm{H}_{31} \mathrm{~N}_{2} \mathrm{O}_{2}$ $[\mathrm{M}+\mathrm{H}]^{+}:$391.2380. Found: 391.2386. 


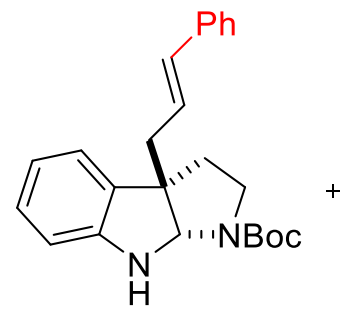

31

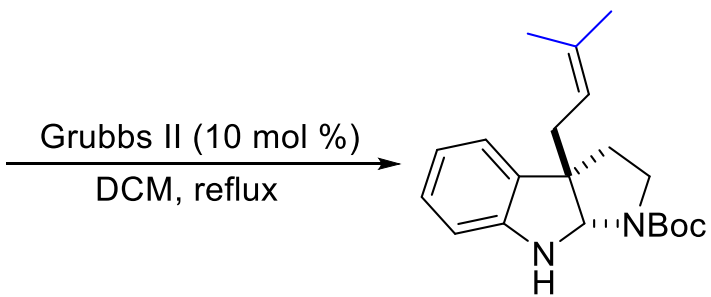

7

To a flame-dried Schlenk tube were added 31 (37.7 mg, 0.1 mmol, 1 equiv), $\mathrm{CH}_{2} \mathrm{Cl}_{2}$ (2 $\mathrm{mL}$ ) and 2-methyl-2-butene (55 ul, $0.5 \mathrm{mmol}, 5$ equiv) under argon. Then the mixture was heated to $40{ }^{\circ} \mathrm{C}$ before Grubbs II $(8.5 \mathrm{mg}, 0.01 \mathrm{mmol}, 10 \mathrm{~mol} \%)$ was added. After the reaction mixture was stirred at $40{ }^{\circ} \mathrm{C}$ for $24 \mathrm{~h}$, additional 2-methyl-2-butene ( $55 \mathrm{ul}, 0.5 \mathrm{mmol}, 5$ equiv) was added. The reaction mixture was further stirred at $40{ }^{\circ} \mathrm{C}$ for $48 \mathrm{~h}$. After the reaction was complete (monitored by TLC), the reaction was quenched by addition of water $(20 \mathrm{~mL})$. The mixture was extracted with EtOAc $(3 \mathrm{x}$ $15 \mathrm{~mL}$ ). The combined extracts were dried over $\mathrm{Na}_{2} \mathrm{SO}_{4}$, and evaporated under reduced pressure. The residue was purified by silica gel column chromatography $(\mathrm{PE} / \mathrm{EtOAc}=9 / 1)$ to afford the desired product 7.

7, dark oil. $25.4 \mathrm{mg}, 77 \%$ yield. ${ }^{1} \mathrm{H}$ NMR (400 MHz, $\mathrm{CDCl}_{3}$ ) (two sets of signals were observed due to the existence of rotamers) $\delta 7.10-7.01(\mathrm{~m}, 2 \mathrm{H}), 6.73(\mathrm{q}, J=7.2 \mathrm{~Hz}$, $1 \mathrm{H}), 6.61$ (d, $J=7.6 \mathrm{~Hz}, 1 \mathrm{H}), 5.22-5.14(\mathrm{~m}, 1 \mathrm{H}), 5.07,4.99$ (s, 1H), 4.63 (br s, 1H), 3.70-3.62, 3.58-3.50 (m, 1H), 3.07-2.93 (m, 1H), 2.45-2.25 (m, 2H), 2.19-2.04 (m, $2 \mathrm{H}), 1.71(\mathrm{~s}, 3 \mathrm{H}), 1.52(\mathrm{~s}, 7 \mathrm{H}), 1.44(\mathrm{~s}, 5 \mathrm{H}) .{ }^{13} \mathrm{C} \mathrm{NMR}\left(100 \mathrm{MHz}, \mathrm{CDCl}_{3}\right.$ ) (two sets of signals were observed due to the existence of rotamers) $\delta 154.7,153.6,149.4,149.0$, 135.0, 132.6, 132.4, 128.24, 128.17, 123.3, 123.2, 119.39, 119.36, 119.0, 118.6, 109.3, $109.2,80.1,80.0,79.9,79.7,58.0,57.0,46.0,45.5,35.9,35.8,34.4,28.8,28.6,26.1$, 18.0. IR (thin film): $v_{\max }\left(\mathrm{cm}^{-1}\right)=3393,3365,2970,2929,2878,2348,2297,2284$, 1681, 1608, 1481, 1466, 1392, 1365, 1311, 1248, 1206, 1160, 1110, 1045, 982, 884, 847, 772, 740, 697, 646, 595, 539, 498, 452. HRMS-ESI calcd for $\mathrm{C}_{20} \mathrm{H}_{29} \mathrm{~N}_{2} \mathrm{O}_{2}$ $[\mathrm{M}+\mathrm{H}]^{+}:$329.2224. Found: 329.2224. 


\section{X-Ray crystal structure of $\mathbf{3 g}$}

\section{CCDC 1955597}

The crystal was obtained by slow evaporation of solution $(\mathrm{DCM} / \mathrm{PE}=10 / 1)$ of $\mathbf{3 g}$

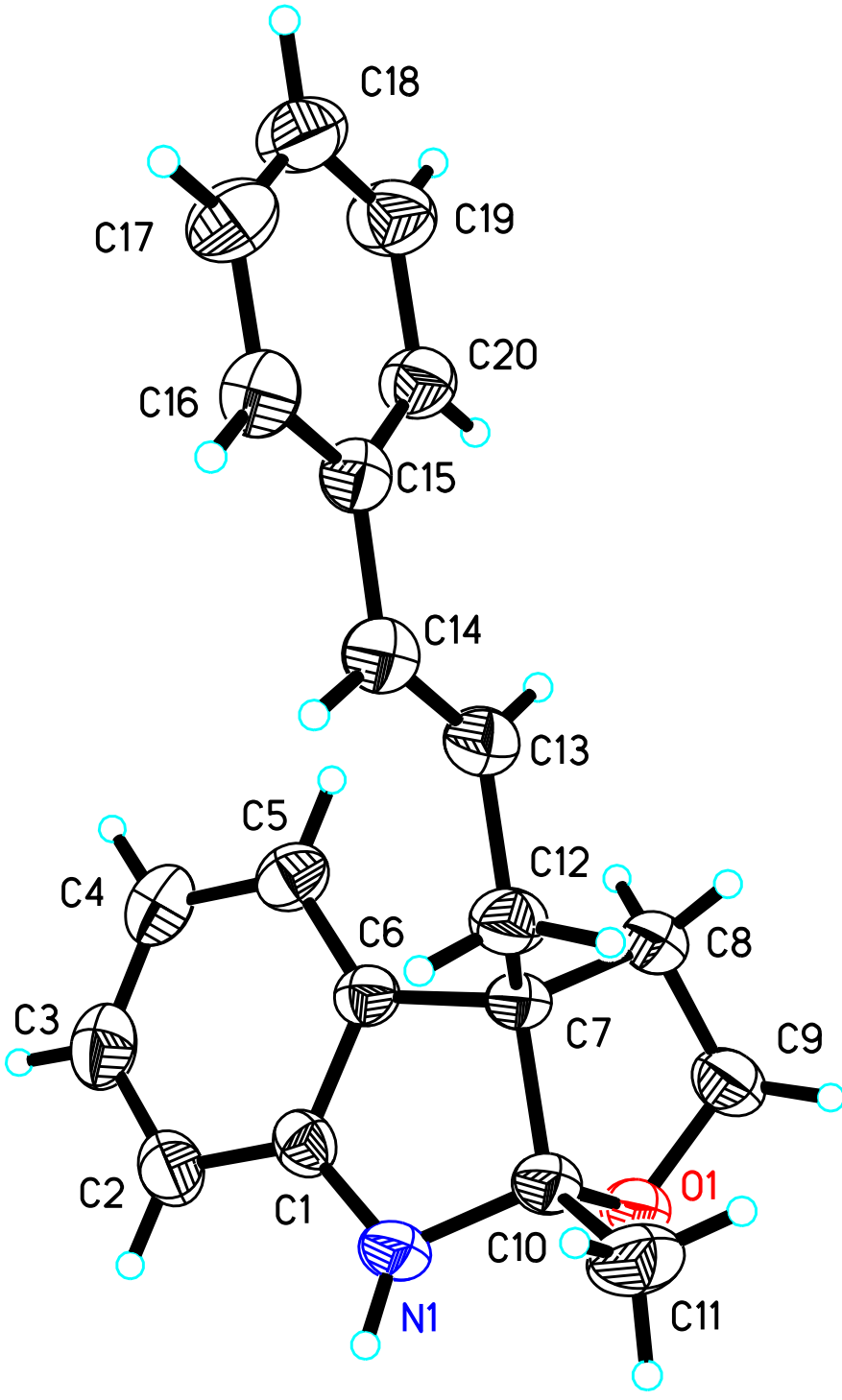

Table S3. Crystal data and structure refinement for d8v19297.

Identification code

Empirical formula d8v19297

$\mathrm{C} 20 \mathrm{H} 21 \mathrm{~N} \mathrm{O}$ 
Formula weight

Temperature

Wavelength

Crystal system

Space group

Unit cell dimensions

Volume

Z

Density (calculated)

Absorption coefficient

$\mathrm{F}(000)$

Crystal size

Theta range for data collection

Index ranges

Reflections collected

Independent reflections

Completeness to theta $=25.242^{\circ}$

Absorption correction

Max. and min. transmission

Refinement method

Data / restraints / parameters

Goodness-of-fit on $\mathrm{F}^{2}$

Final R indices [I $>2 \operatorname{sigma}(\mathrm{I})]$

$\mathrm{R}$ indices (all data)

Extinction coefficient

Largest diff. peak and hole
291.38

293(2) K

$0.71073 \AA$

Monoclinic

P 21/c

$\begin{array}{ll}\mathrm{a}=8.5372(13) \AA & \alpha=90^{\circ} . \\ \mathrm{b}=24.065(4) \AA & \beta=107.447(3)^{\circ} . \\ \mathrm{c}=8.5300(13) \AA & \gamma=90^{\circ} .\end{array}$

$1671.9(5) \AA^{3}$

4

$1.158 \mathrm{Mg} / \mathrm{m}^{3}$

$0.071 \mathrm{~mm}^{-1}$

624

$0.150 \times 0.100 \times 0.060 \mathrm{~mm}^{3}$

2.501 to $24.999^{\circ}$.

$-10<=\mathrm{h}<=10,-24<=\mathrm{k}<=28,-10<=1<=10$

15412

$2913[\mathrm{R}(\mathrm{int})=0.0728]$

$96.4 \%$

Semi-empirical from equivalents

0.7456 and 0.6230

Full-matrix least-squares on $\mathrm{F}^{2}$

2913 / 0 / 201

1.082

$\mathrm{R} 1=0.0880, \mathrm{wR} 2=0.2333$

$\mathrm{R} 1=0.1398, \mathrm{wR} 2=0.2750$

$0.023(6)$

0.304 and -0.234 e. $\AA^{-3}$ 


\section{Copies of NMR spectra}

${ }^{1} \mathrm{H}$ NMR spectra of $\mathbf{3 a}$

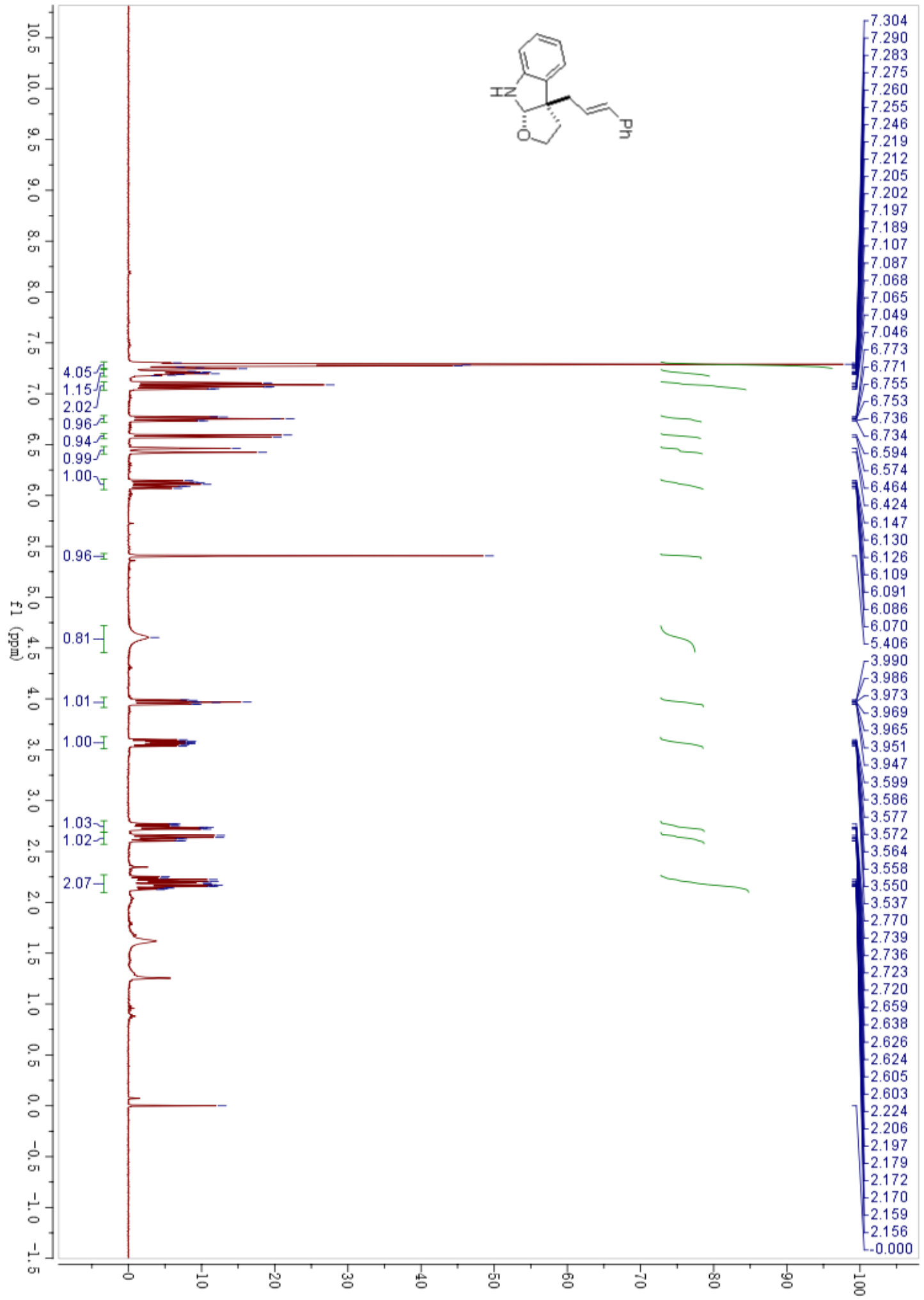


${ }^{1} \mathrm{H}$ NMR spectra of $\mathbf{3 b}$

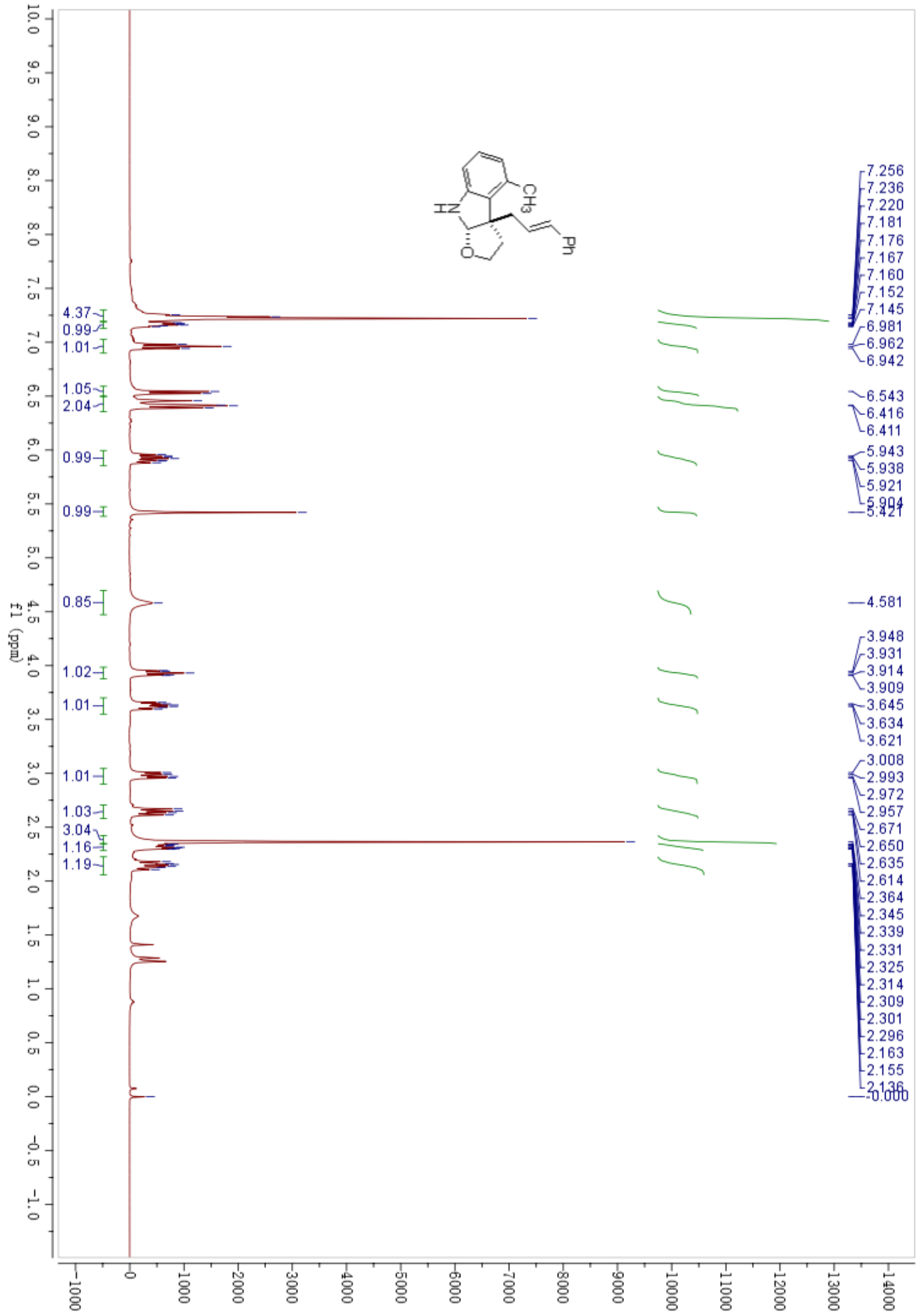


${ }^{13} \mathrm{C}$ NMR spectra of $\mathbf{3 b}$

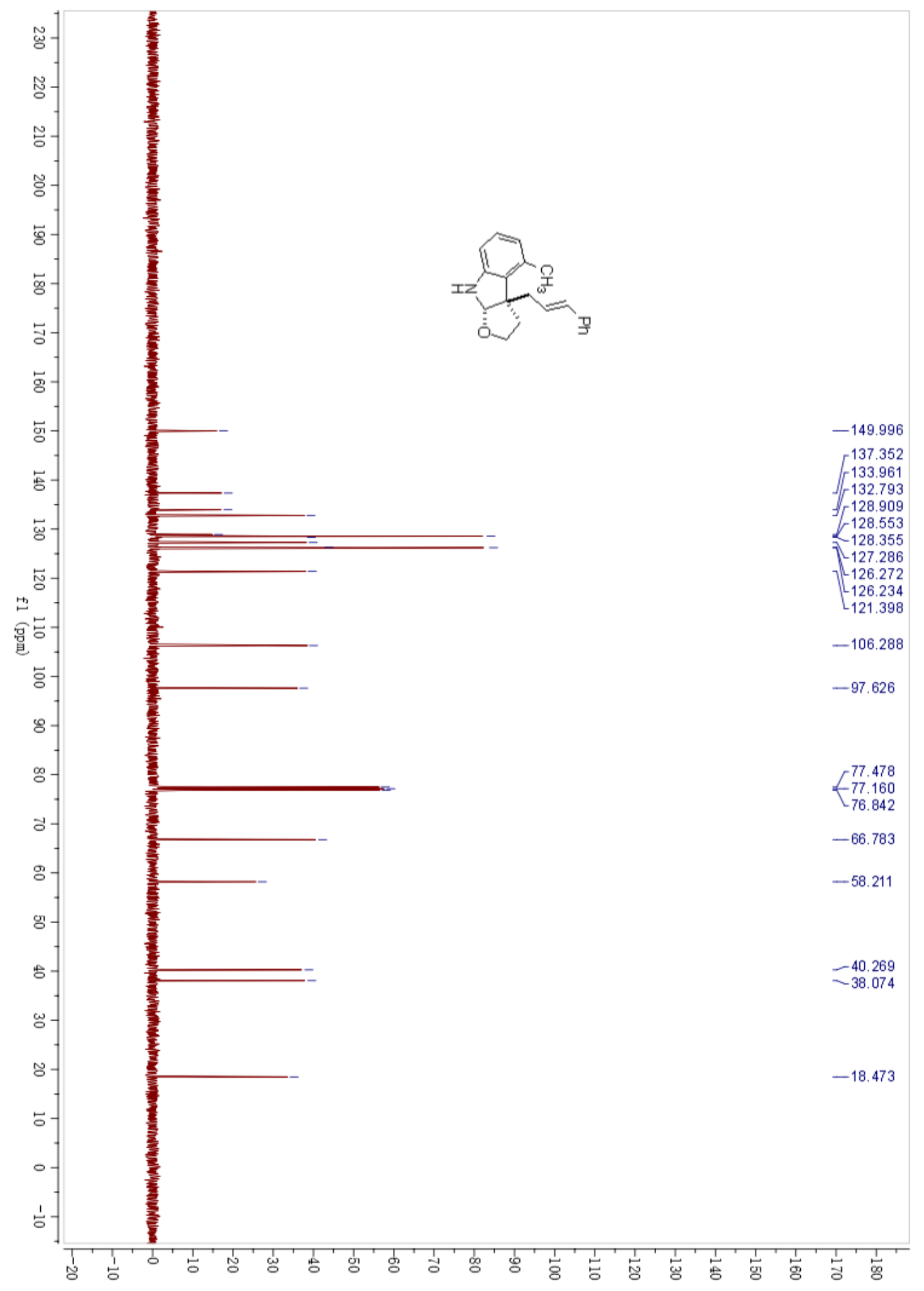


${ }^{1} \mathrm{H}$ NMR spectra of $\mathbf{3 c}$

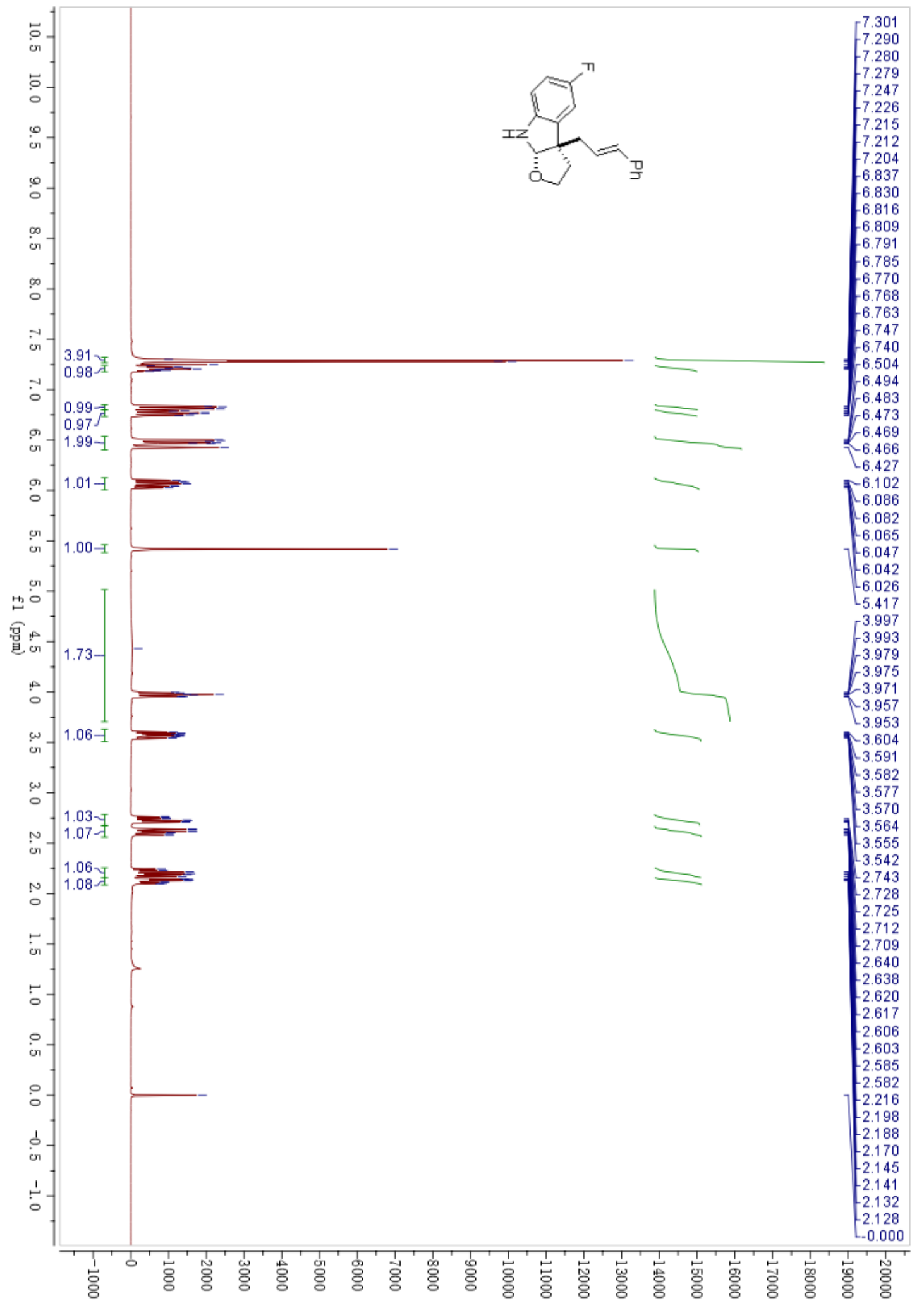


${ }^{13} \mathrm{C}$ NMR spectra of $\mathbf{3 c}$

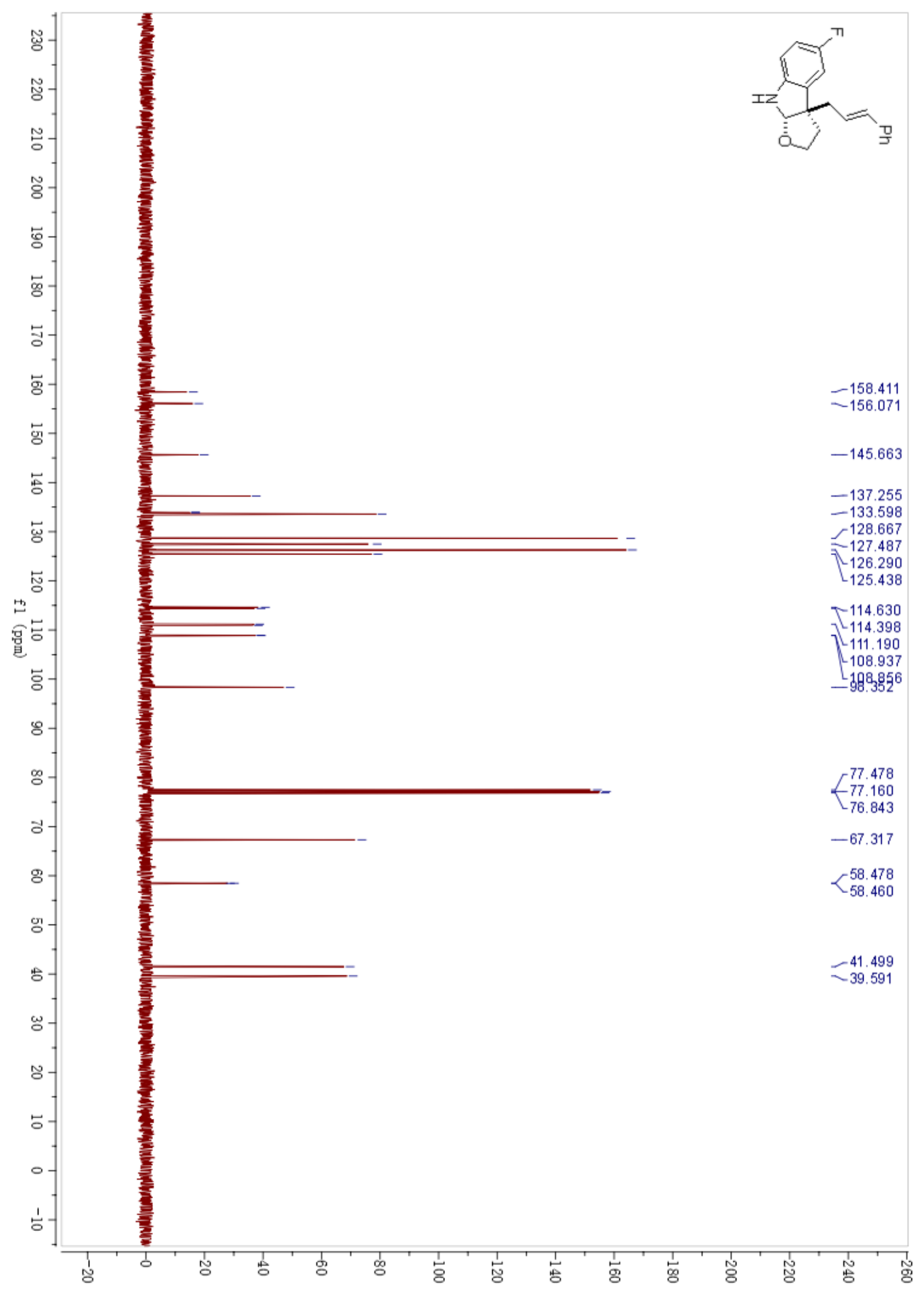


${ }^{19}$ F NMR spectra of $\mathbf{3 c}$

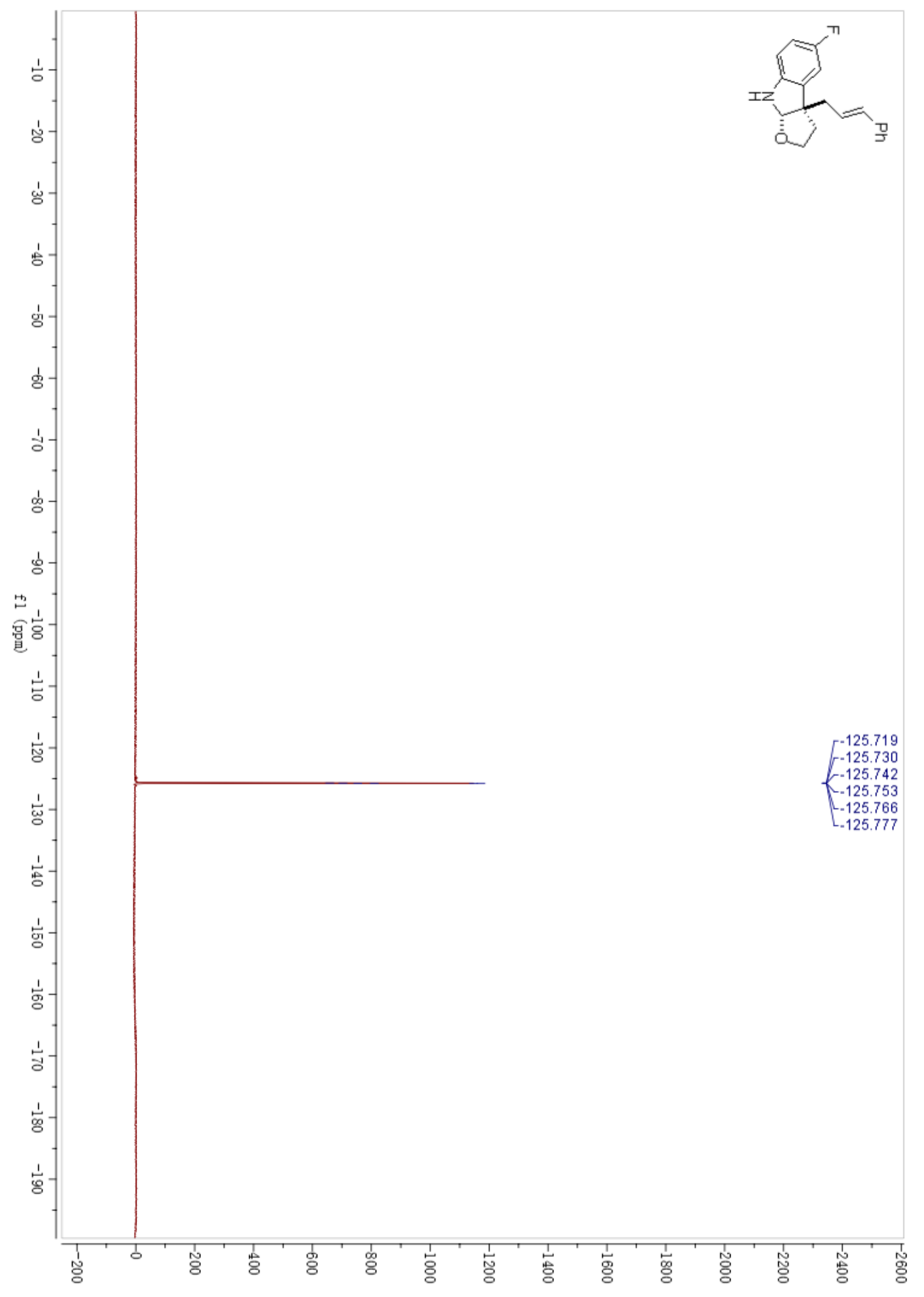


${ }^{1} \mathrm{H}$ NMR spectra of $\mathbf{3 d}$

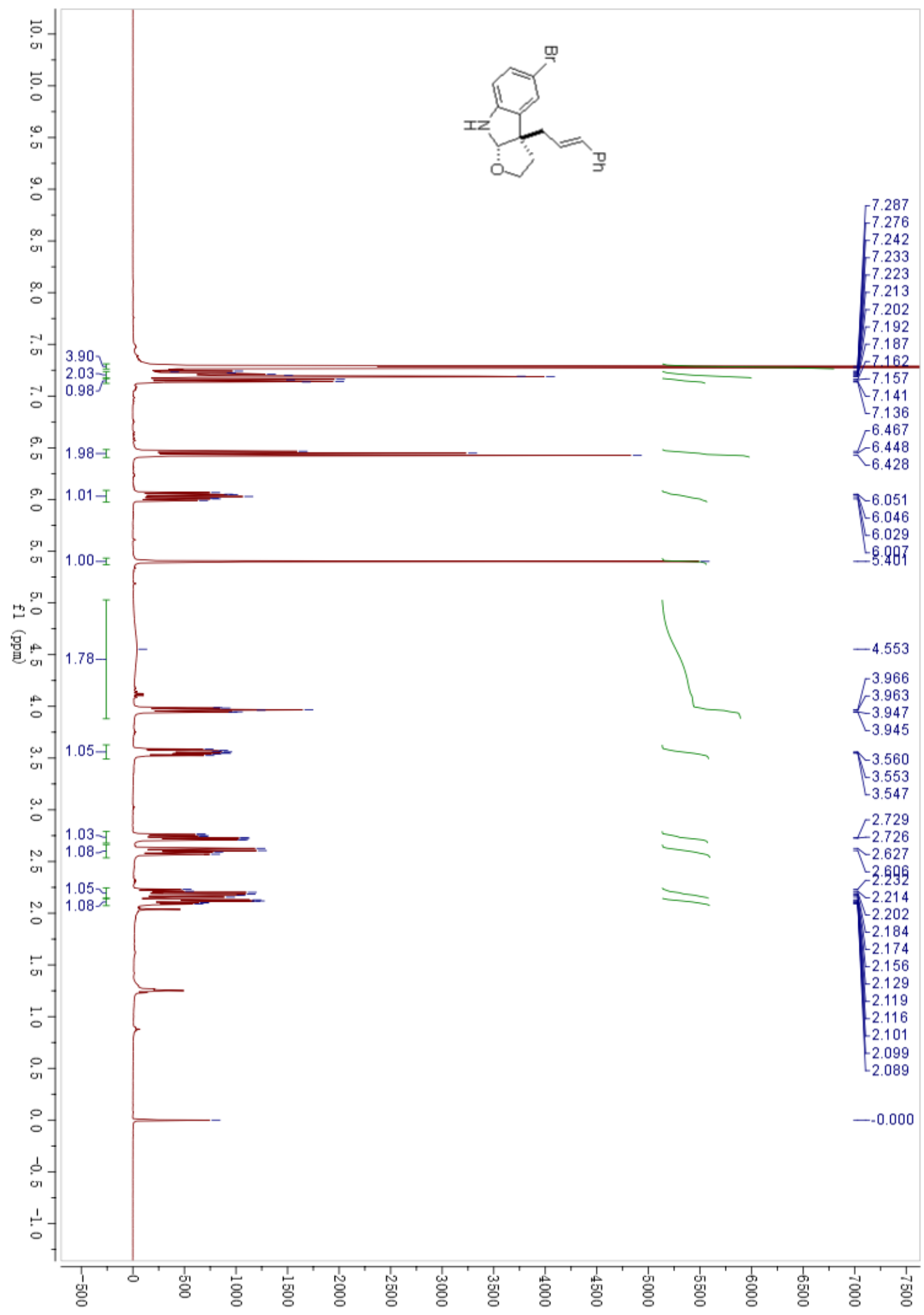


${ }^{13} \mathrm{C}$ NMR spectra of $\mathbf{3 d}$

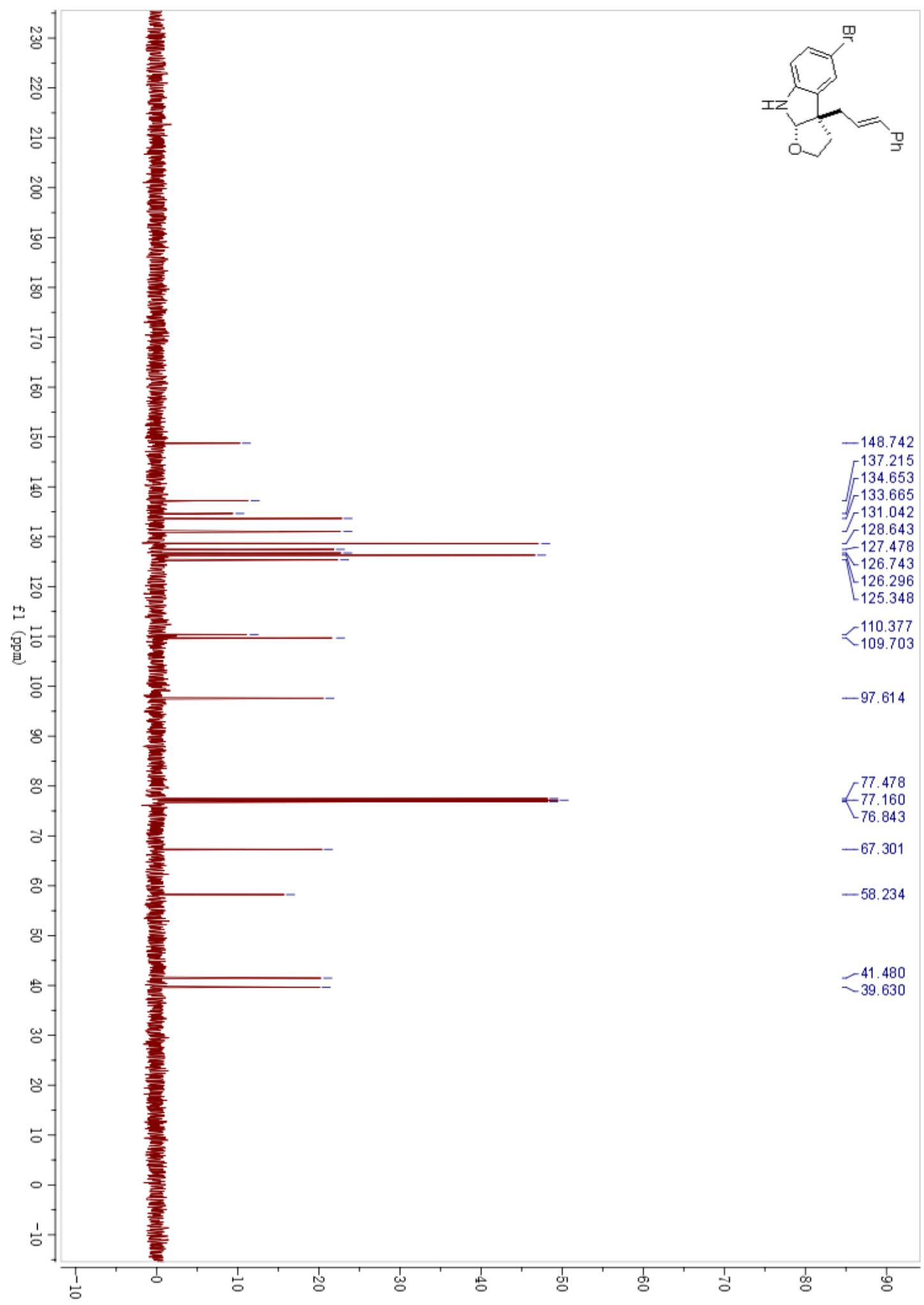


${ }^{1} \mathrm{H}$ NMR spectra of $\mathbf{3 e}$

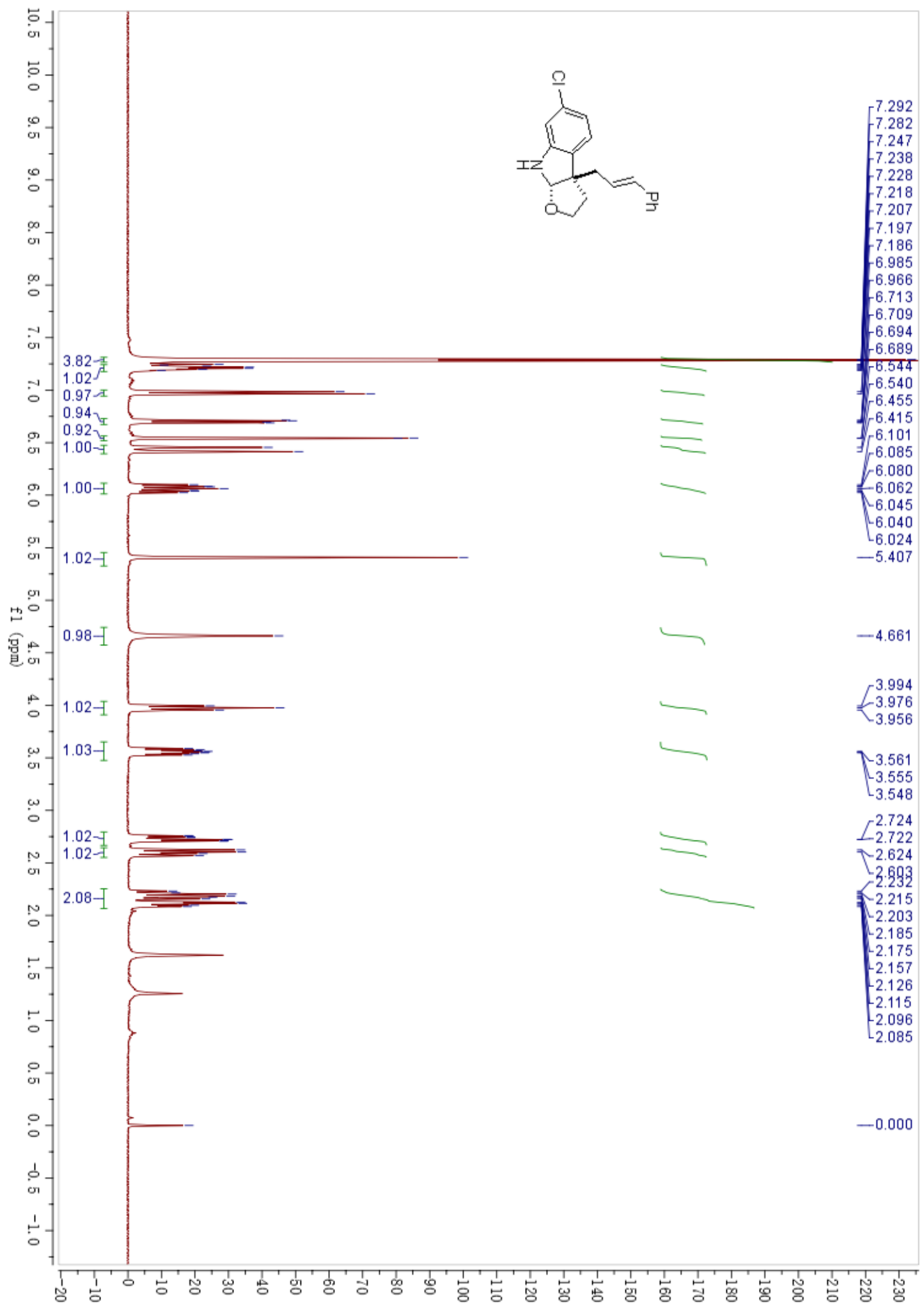


${ }^{13} \mathrm{C}$ NMR spectra of $\mathbf{3 e}$

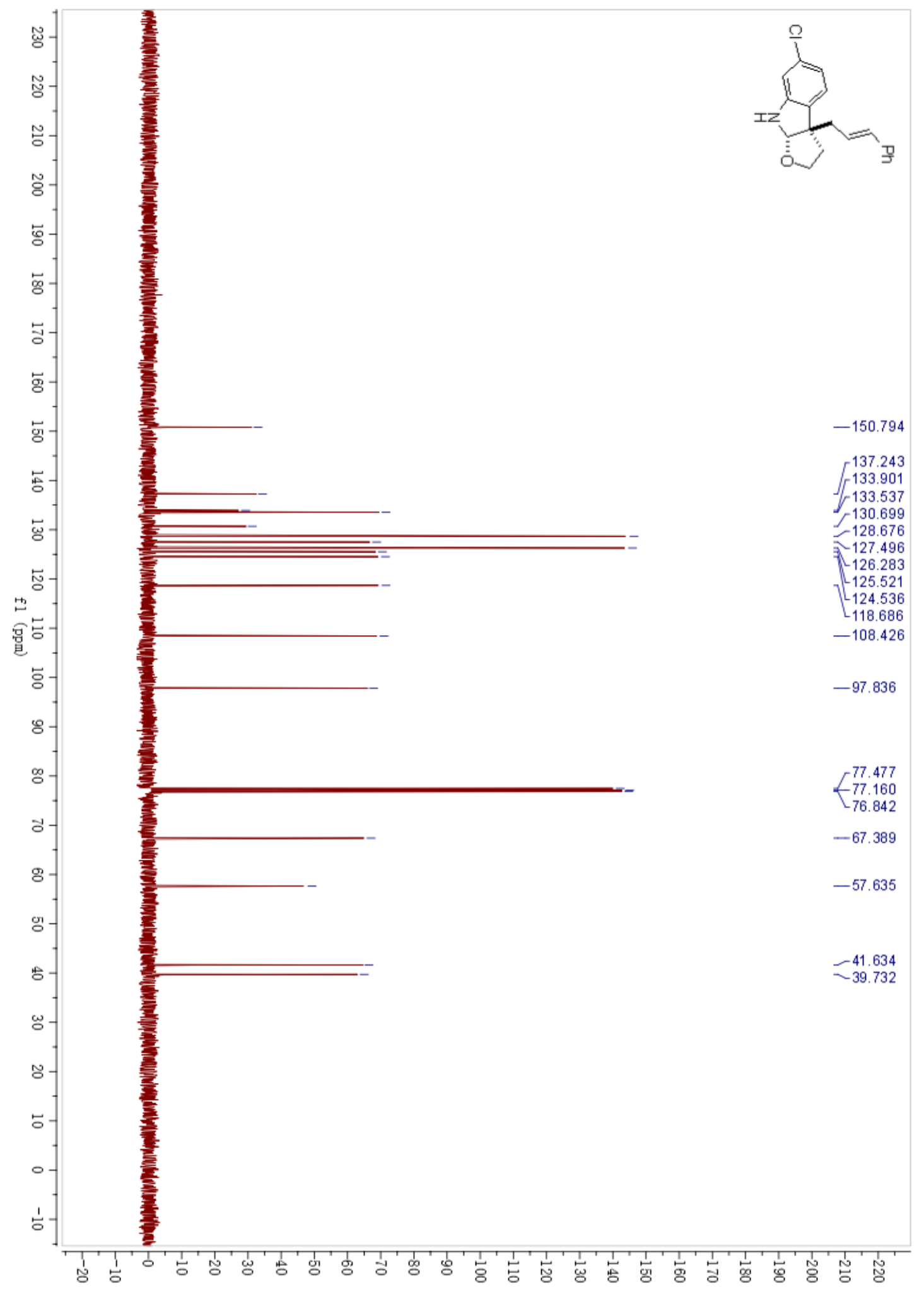


${ }^{1} \mathrm{H}$ NMR spectra of $\mathbf{3 e}{ }^{\prime}$

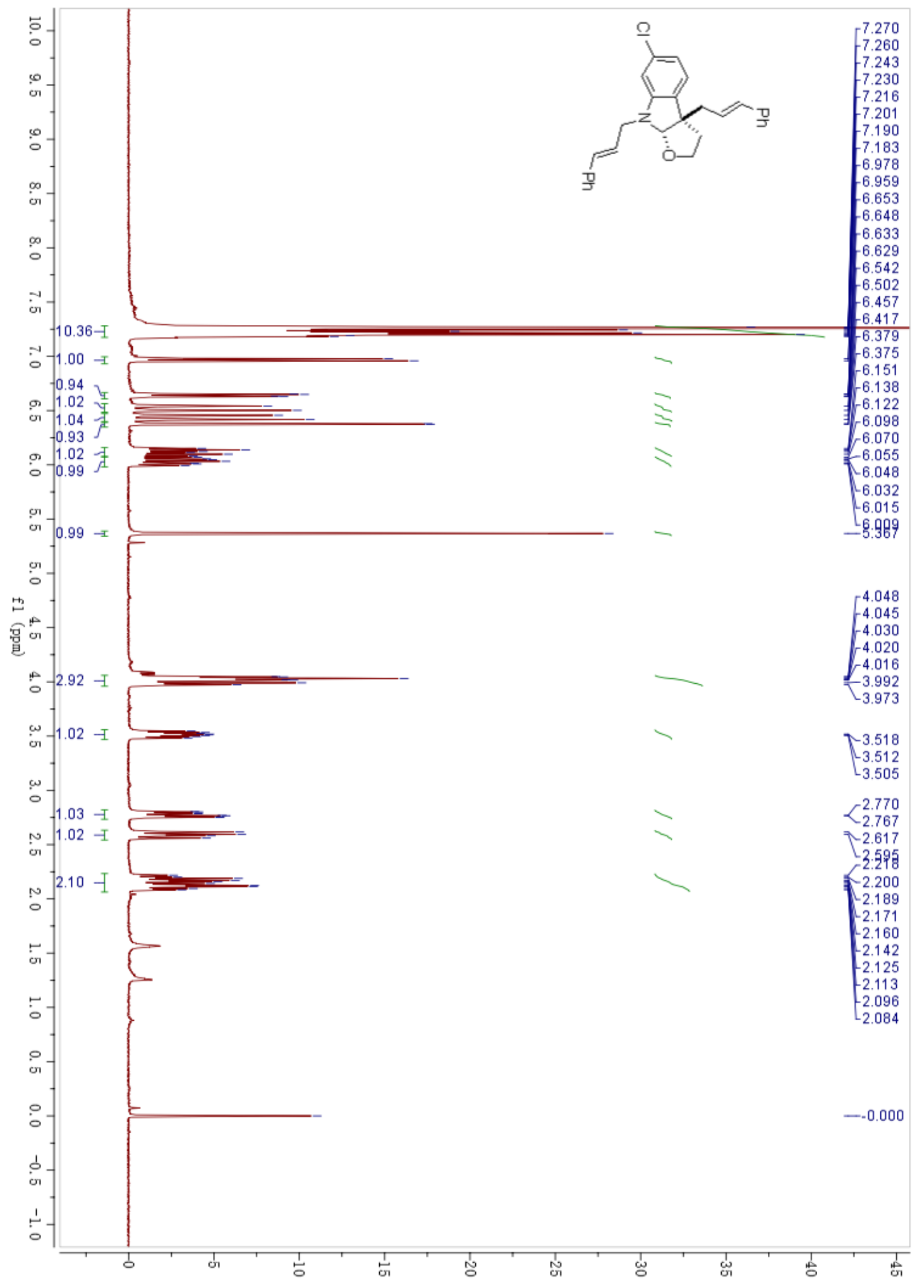


${ }^{13} \mathrm{C}$ NMR spectra of $\mathbf{3 e}$ '

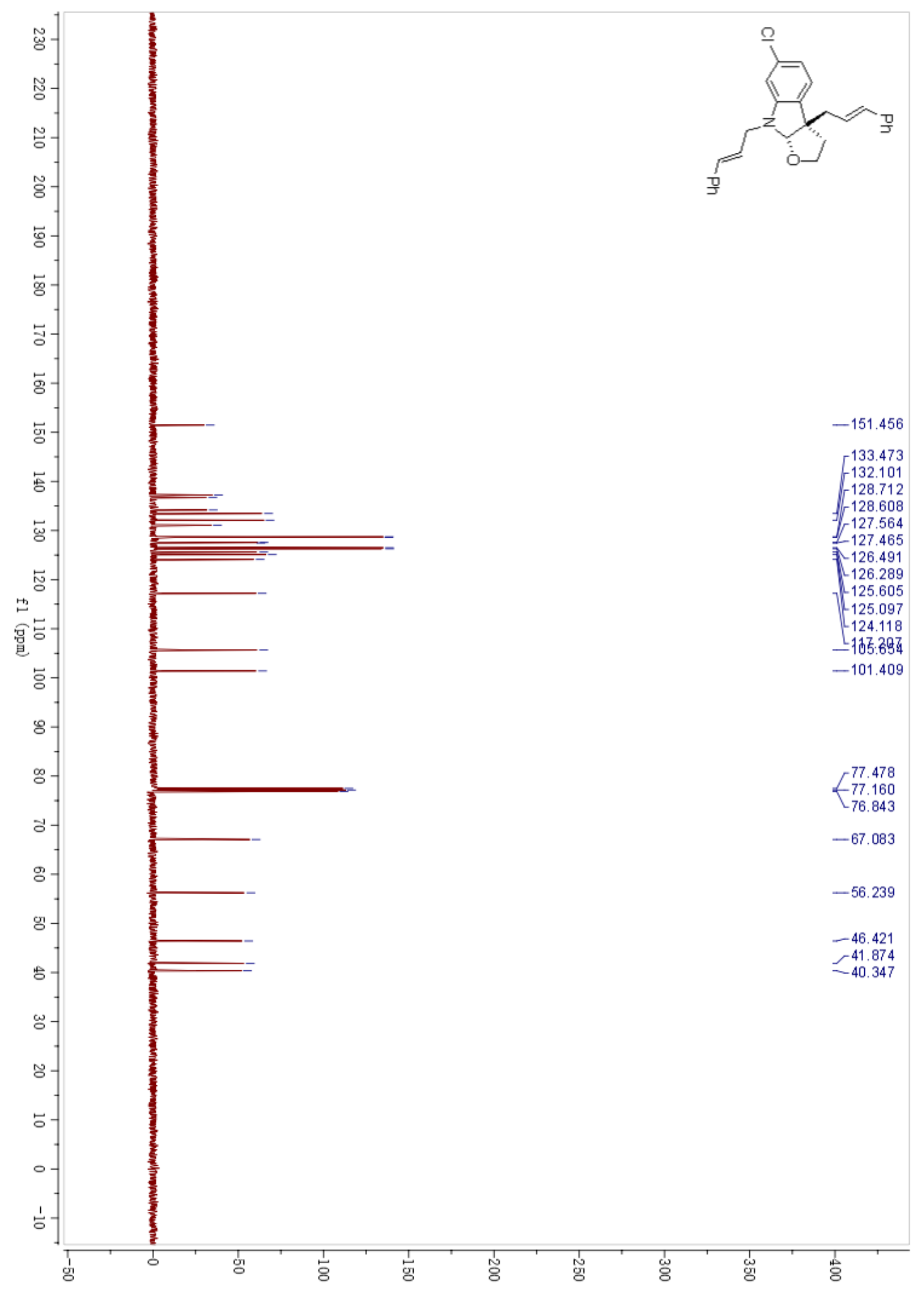


${ }^{1} \mathrm{H}$ NMR spectra of $\mathbf{3 f}$

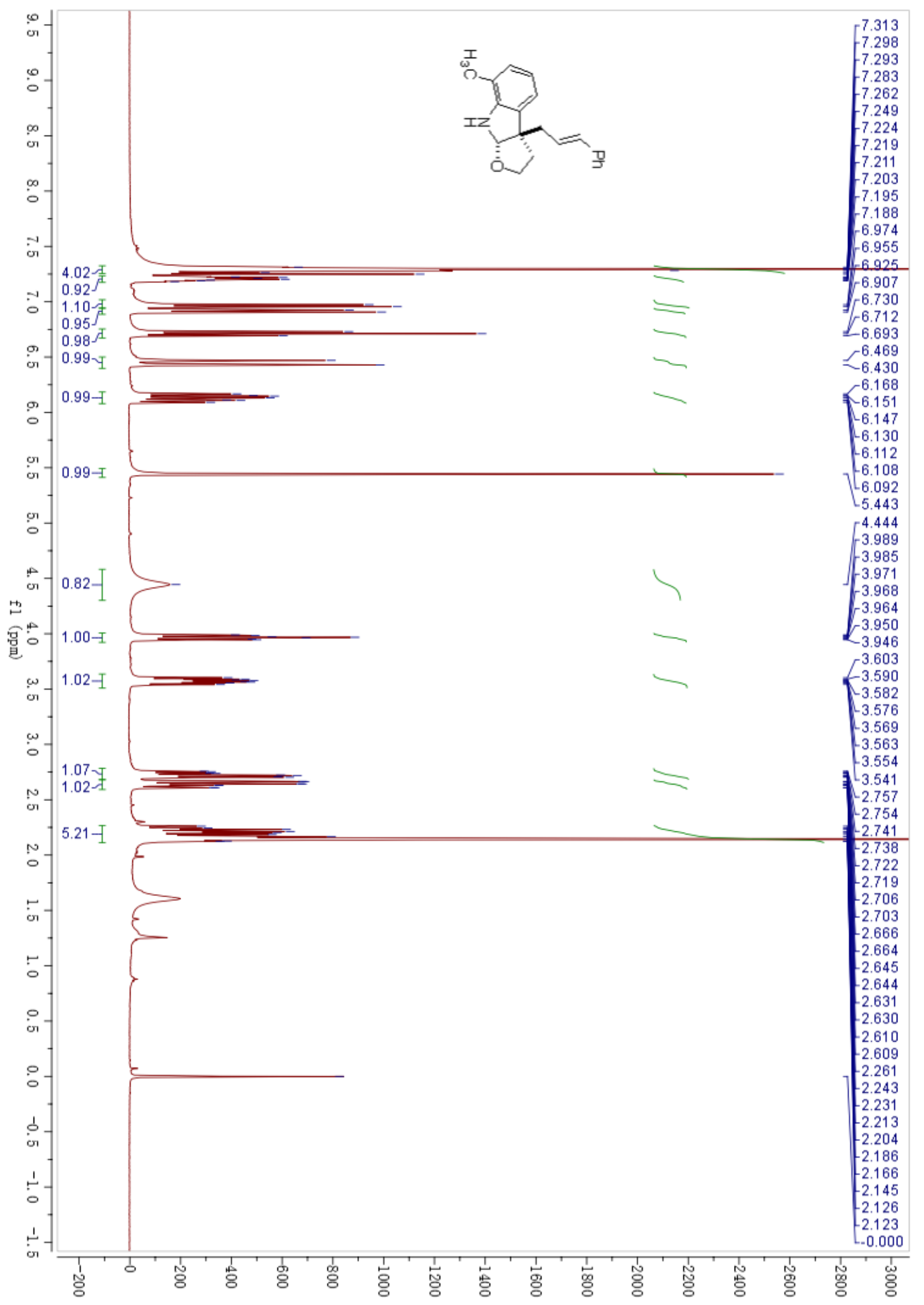


${ }^{13} \mathrm{C}$ NMR spectra of $\mathbf{3 f}$

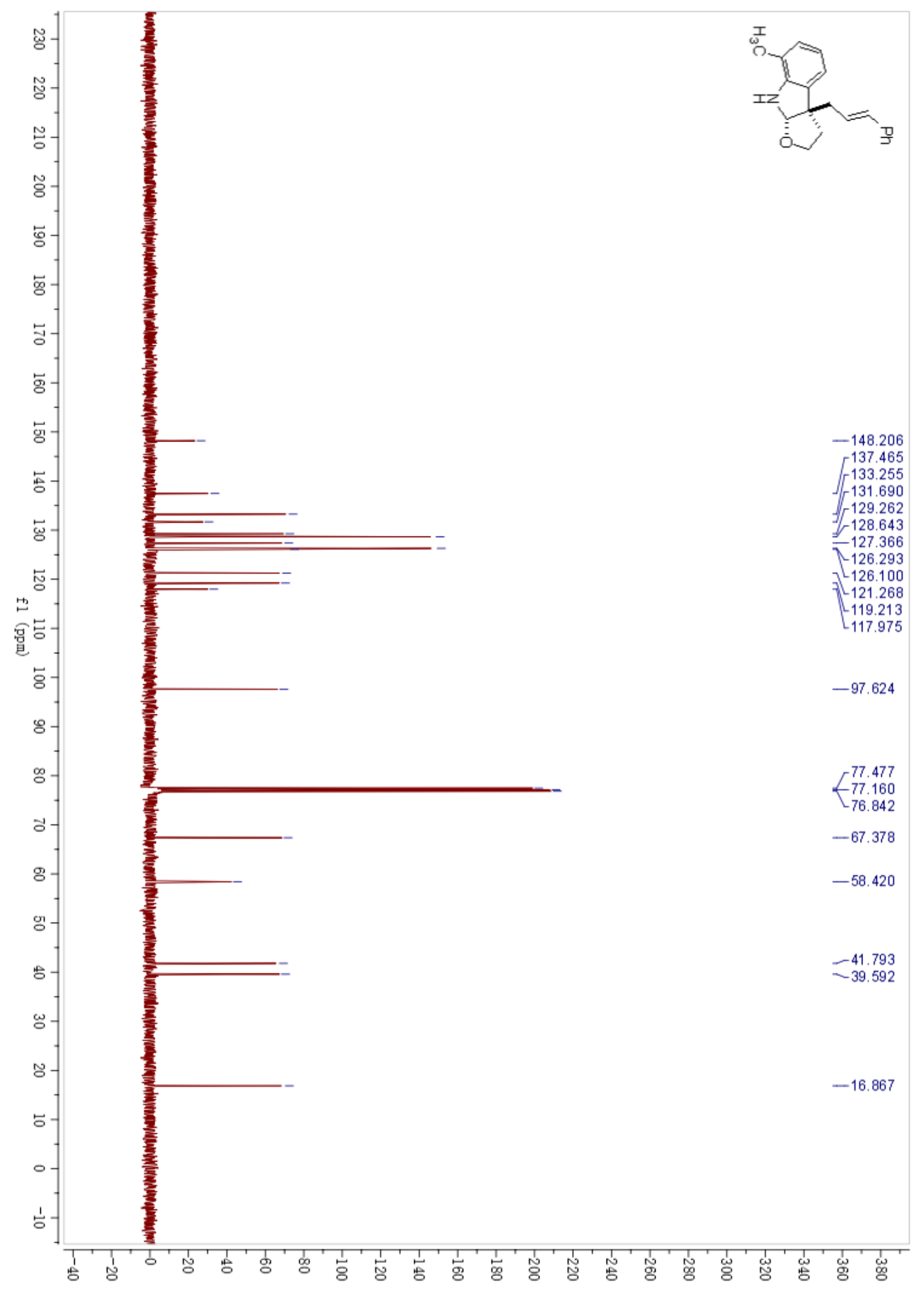


${ }^{1} \mathrm{H}$ NMR spectra of $\mathbf{3 g}$

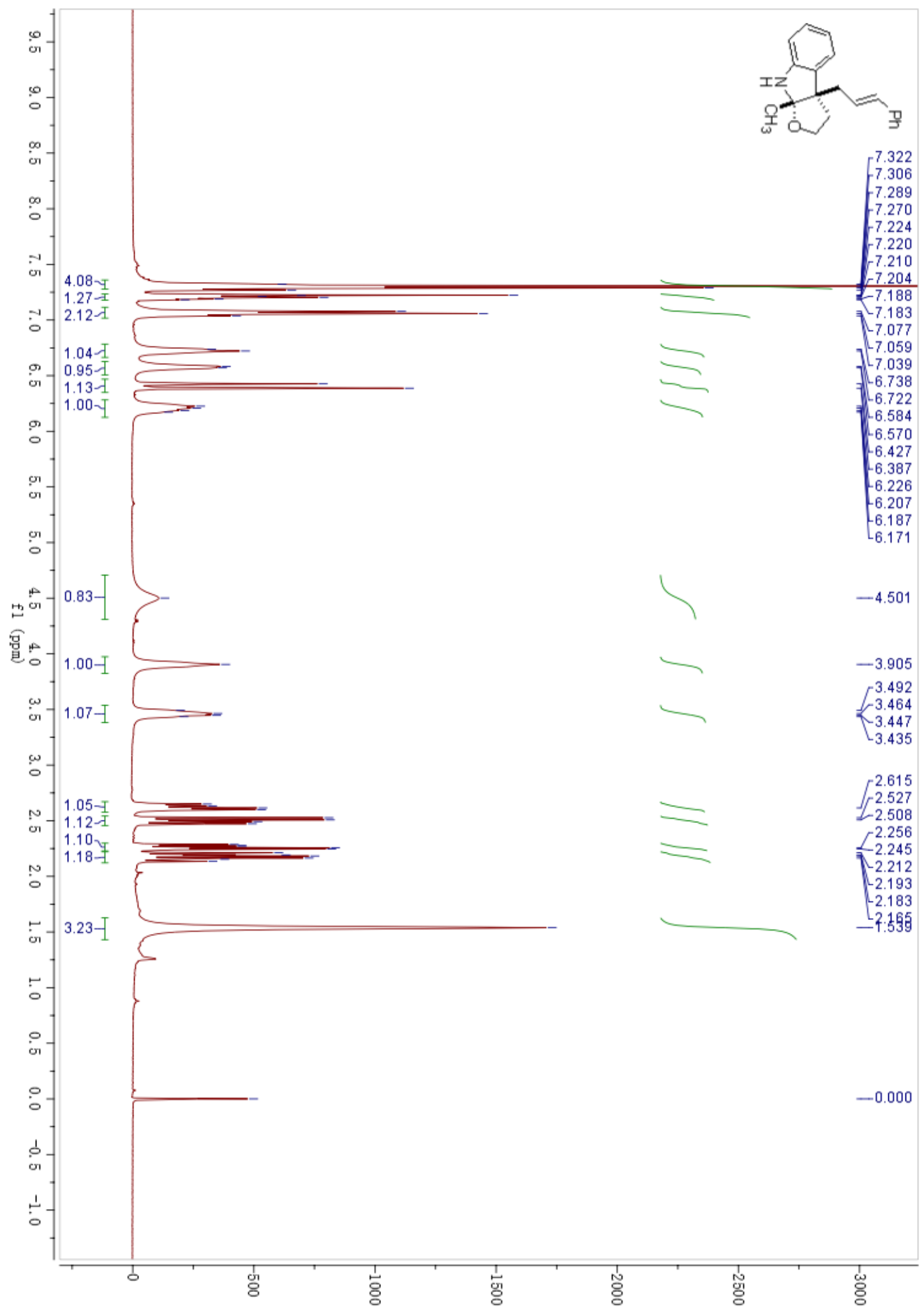


${ }^{13} \mathrm{C}$ NMR spectra of $\mathbf{3 g}$

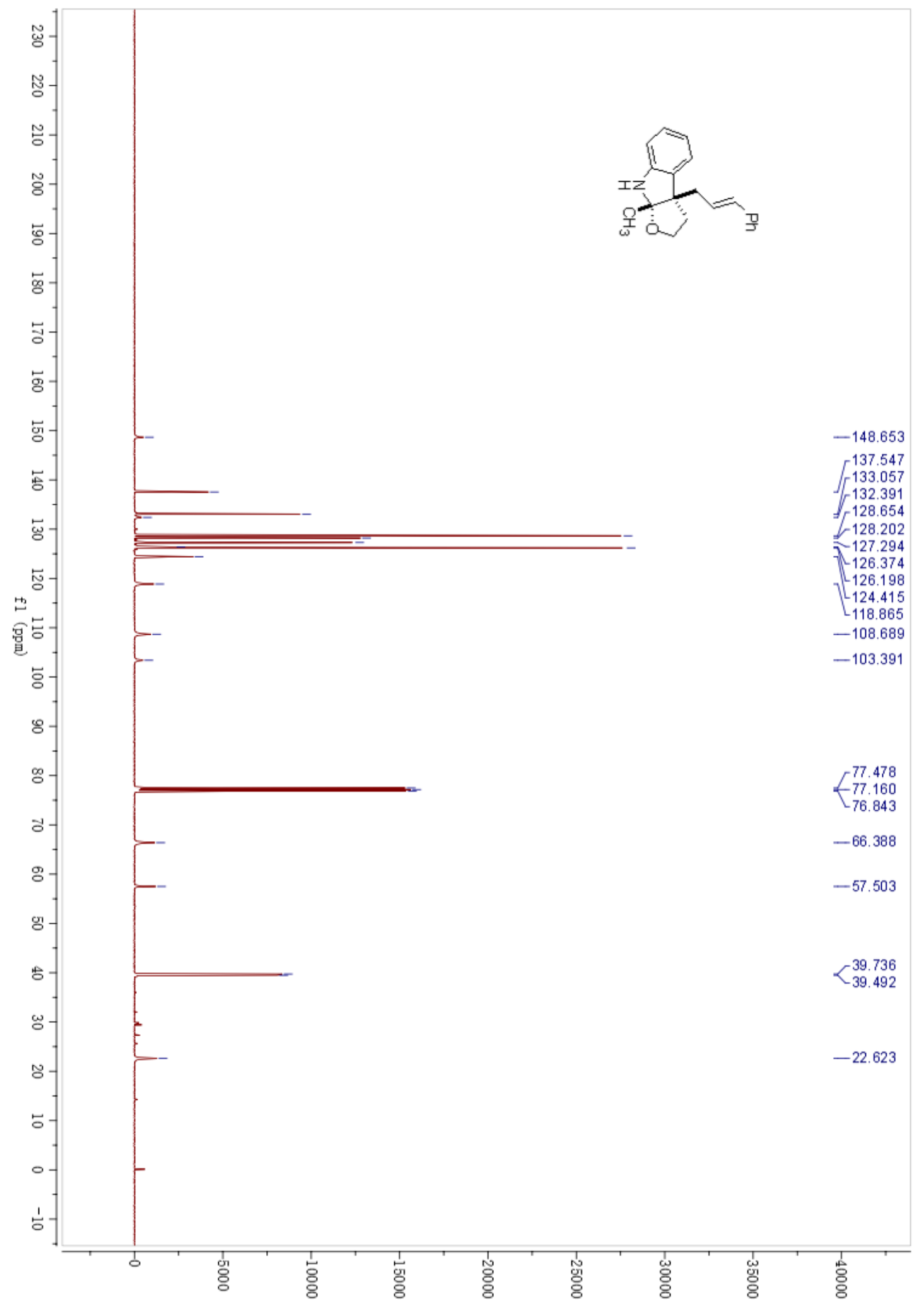


${ }^{1} \mathrm{H}$ NMR spectra of $\mathbf{3 h}$

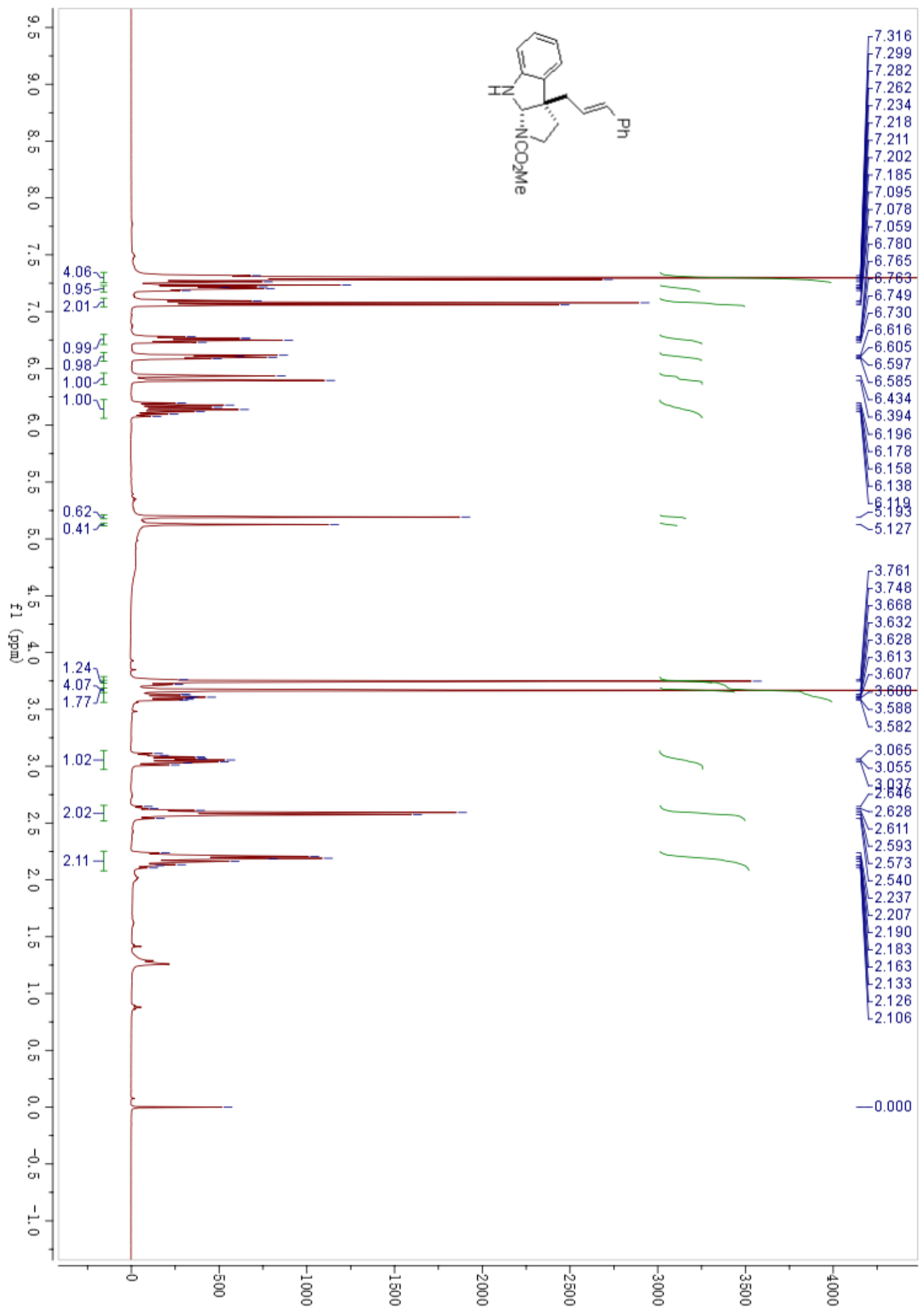


${ }^{1} \mathrm{H}$ NMR spectra of $\mathbf{3 i}$

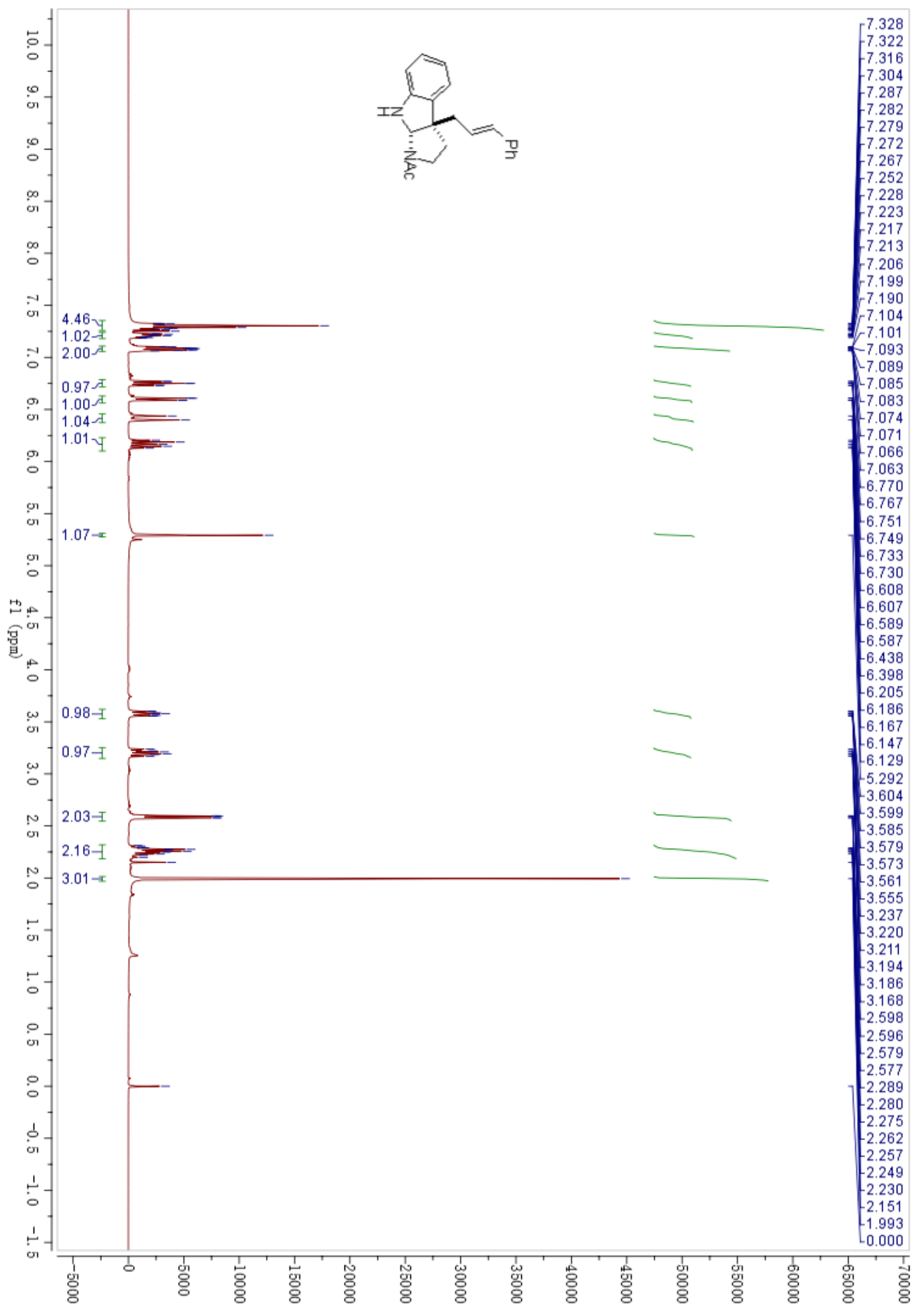


${ }^{13} \mathrm{C}$ NMR spectra of $\mathbf{3 i}$

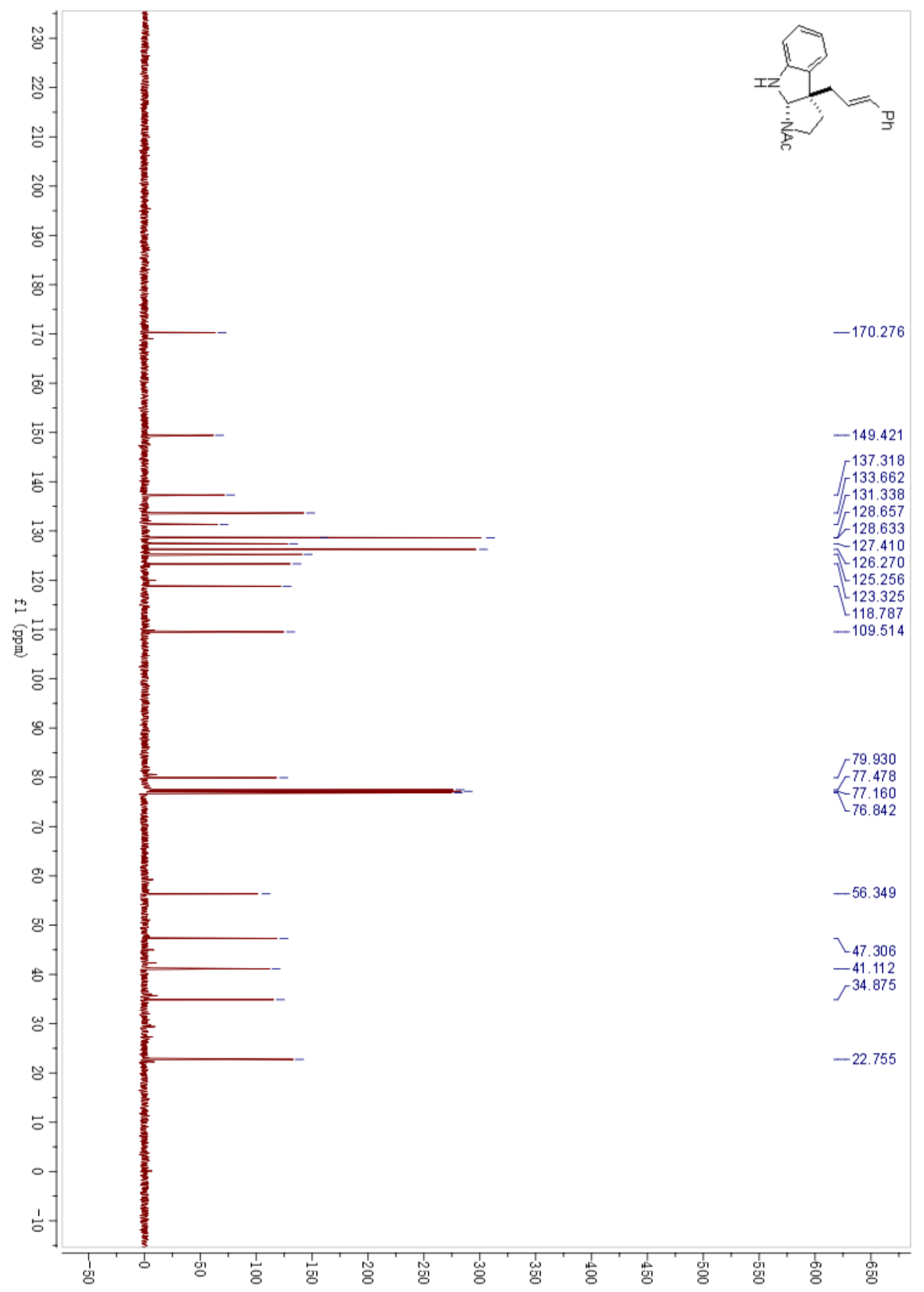


${ }^{1} \mathrm{H}$ NMR spectra of $\mathbf{3 j}$

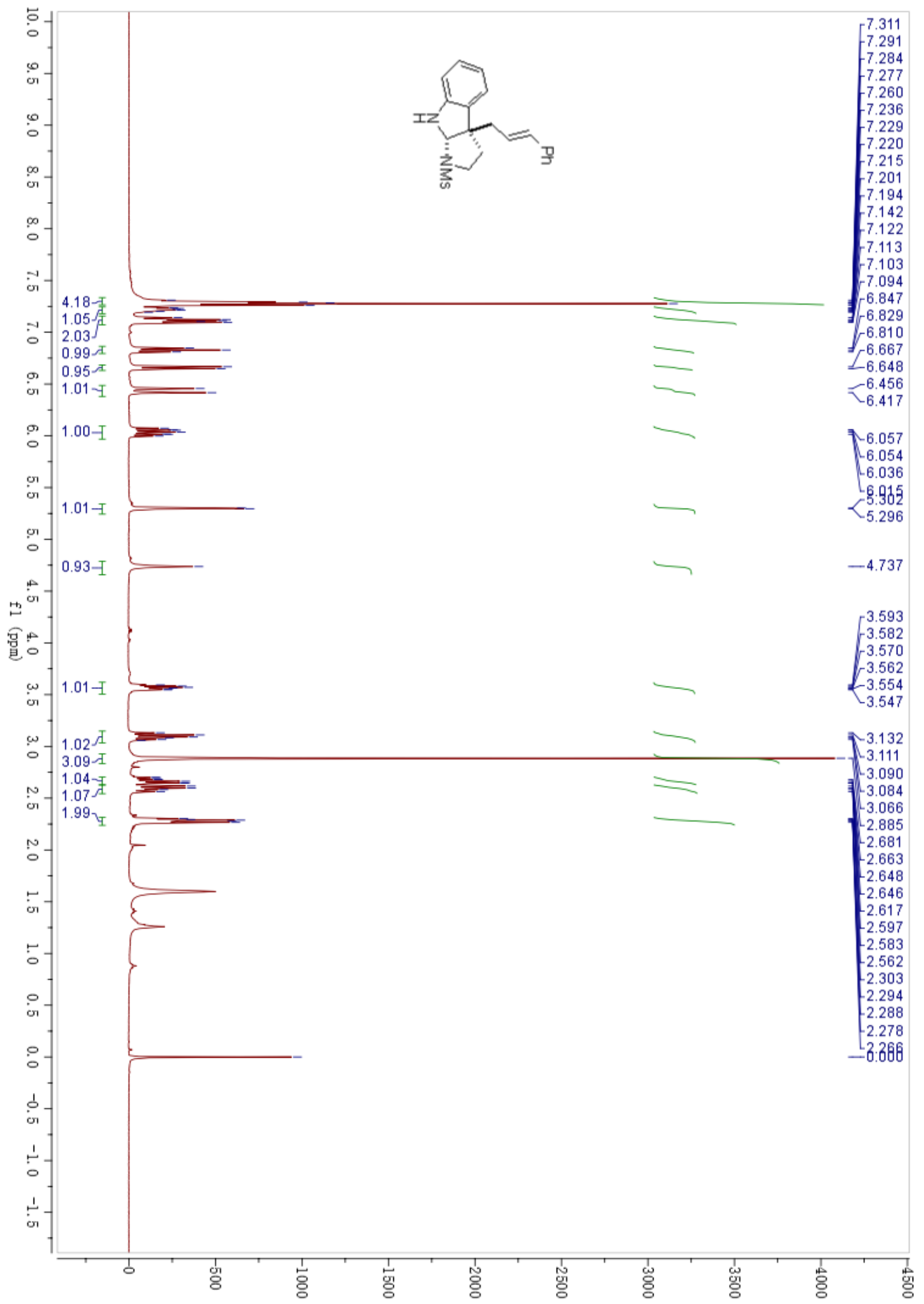


${ }^{13} \mathrm{C}$ NMR spectra of $\mathbf{3 j}$

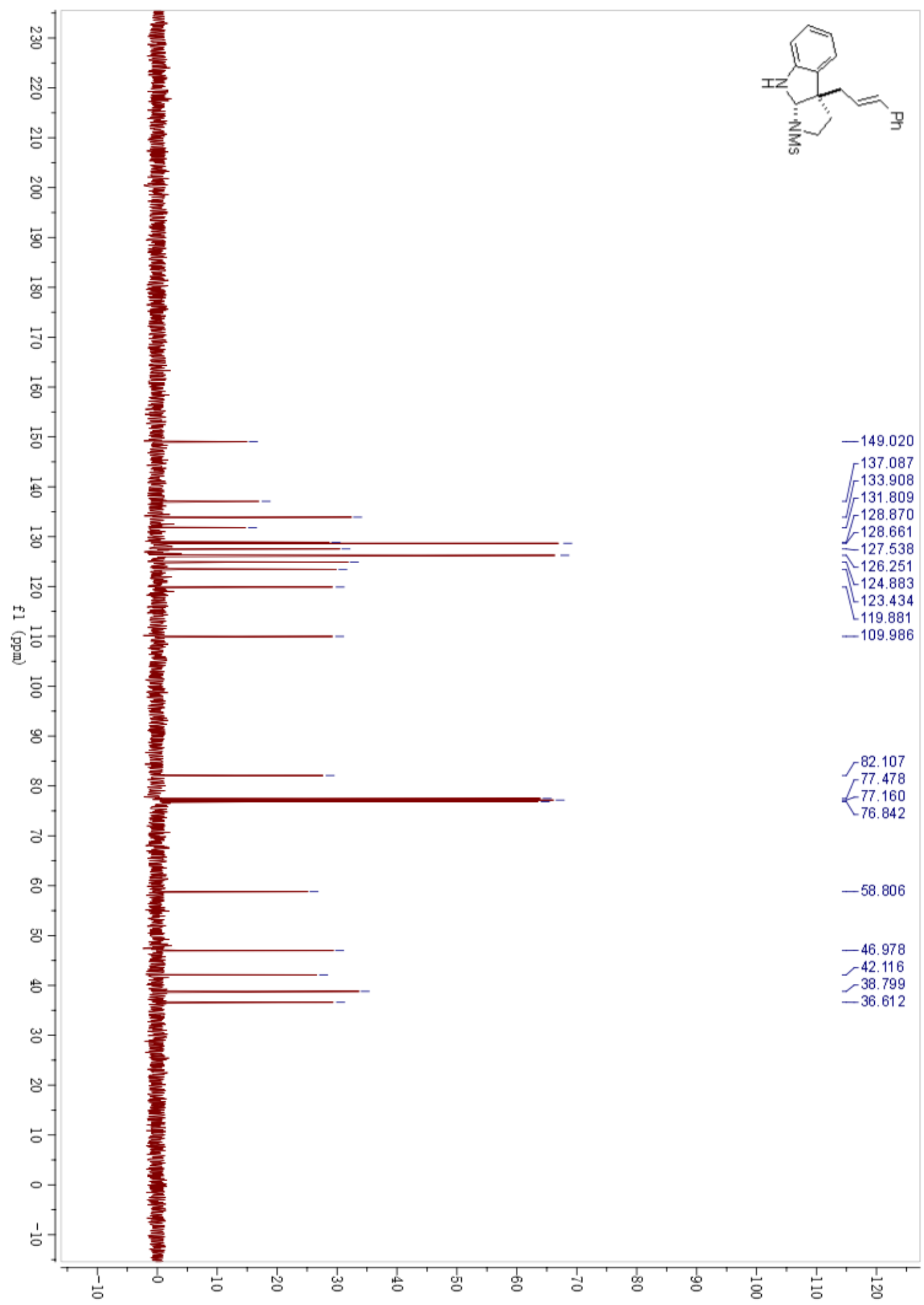


${ }^{1} \mathrm{H}$ NMR spectra of $\mathbf{4 j}$

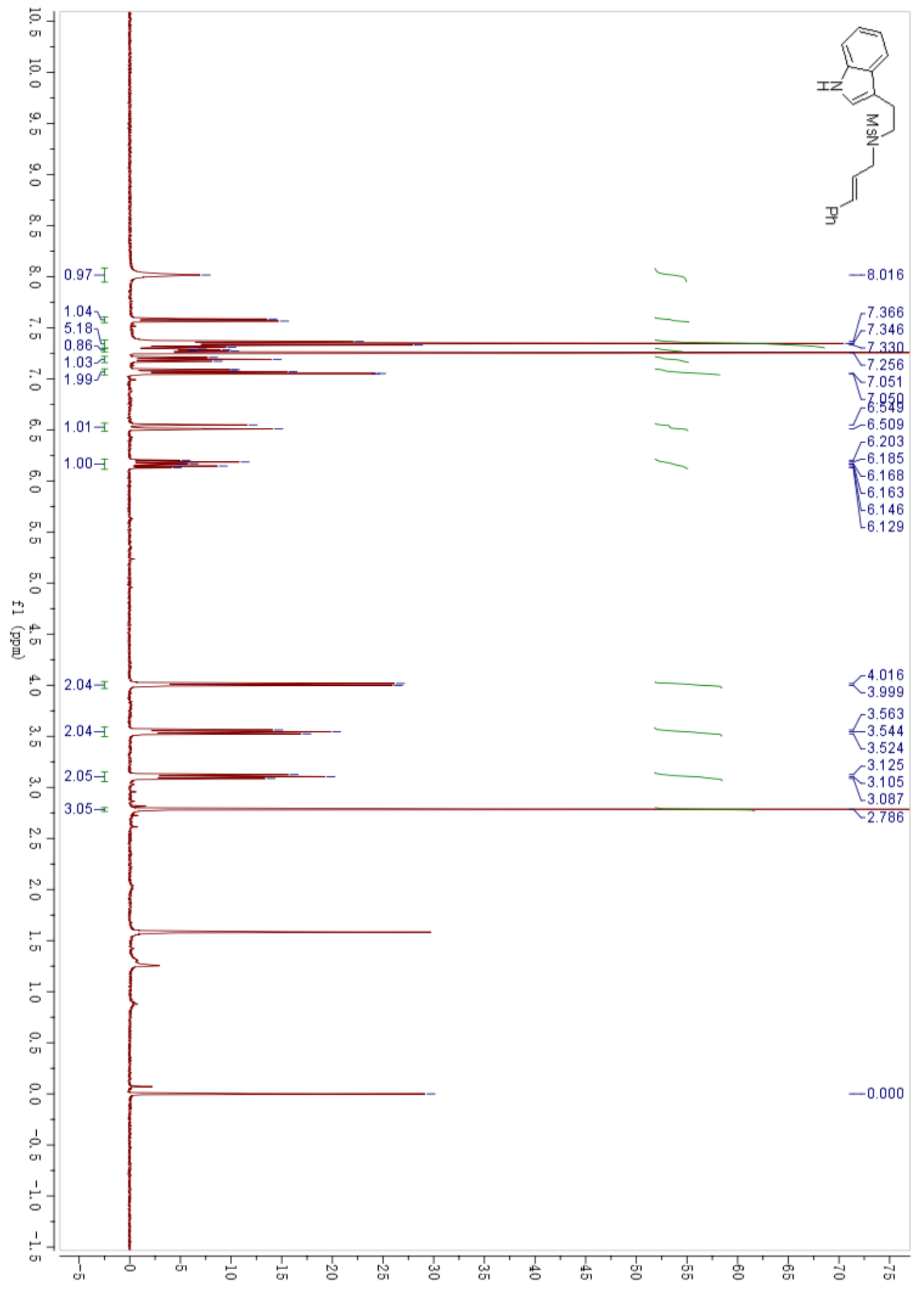


${ }^{13} \mathrm{C}$ NMR spectra of $\mathbf{4} \mathbf{j}$

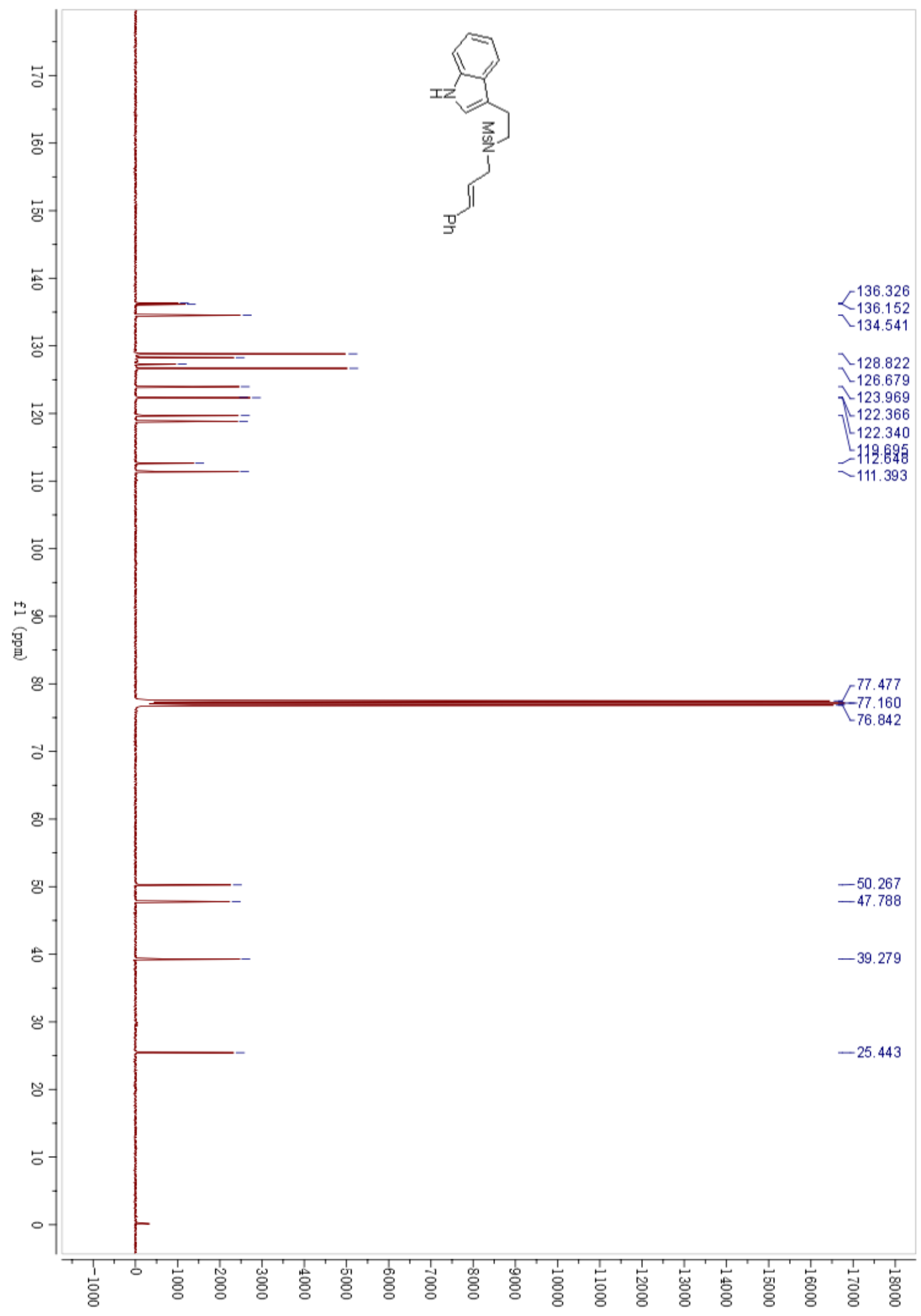


${ }^{1} \mathrm{H}$ NMR spectra of $\mathbf{3 k}$

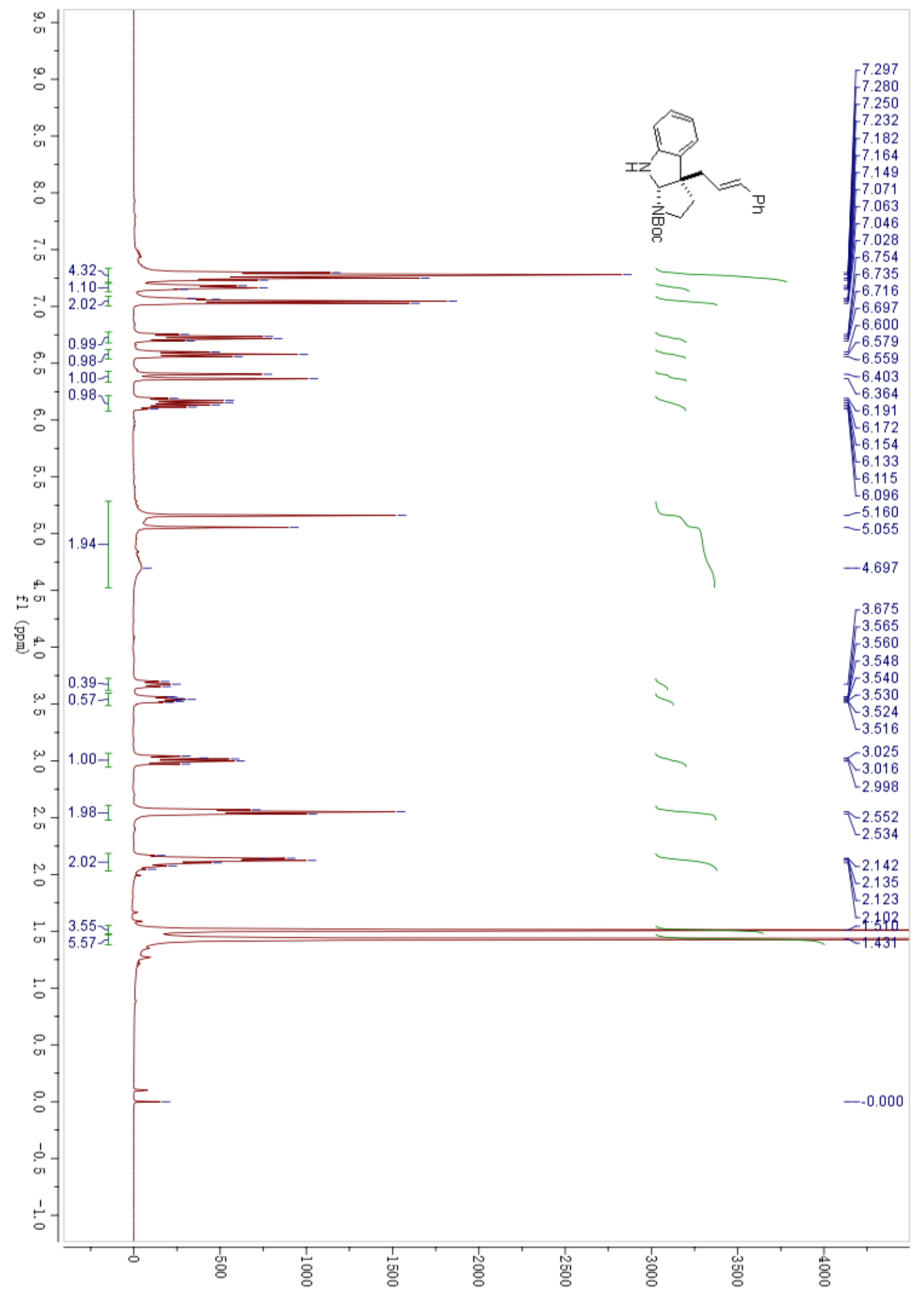


${ }^{1} \mathrm{H}$ NMR spectra of $\mathbf{3 l}$

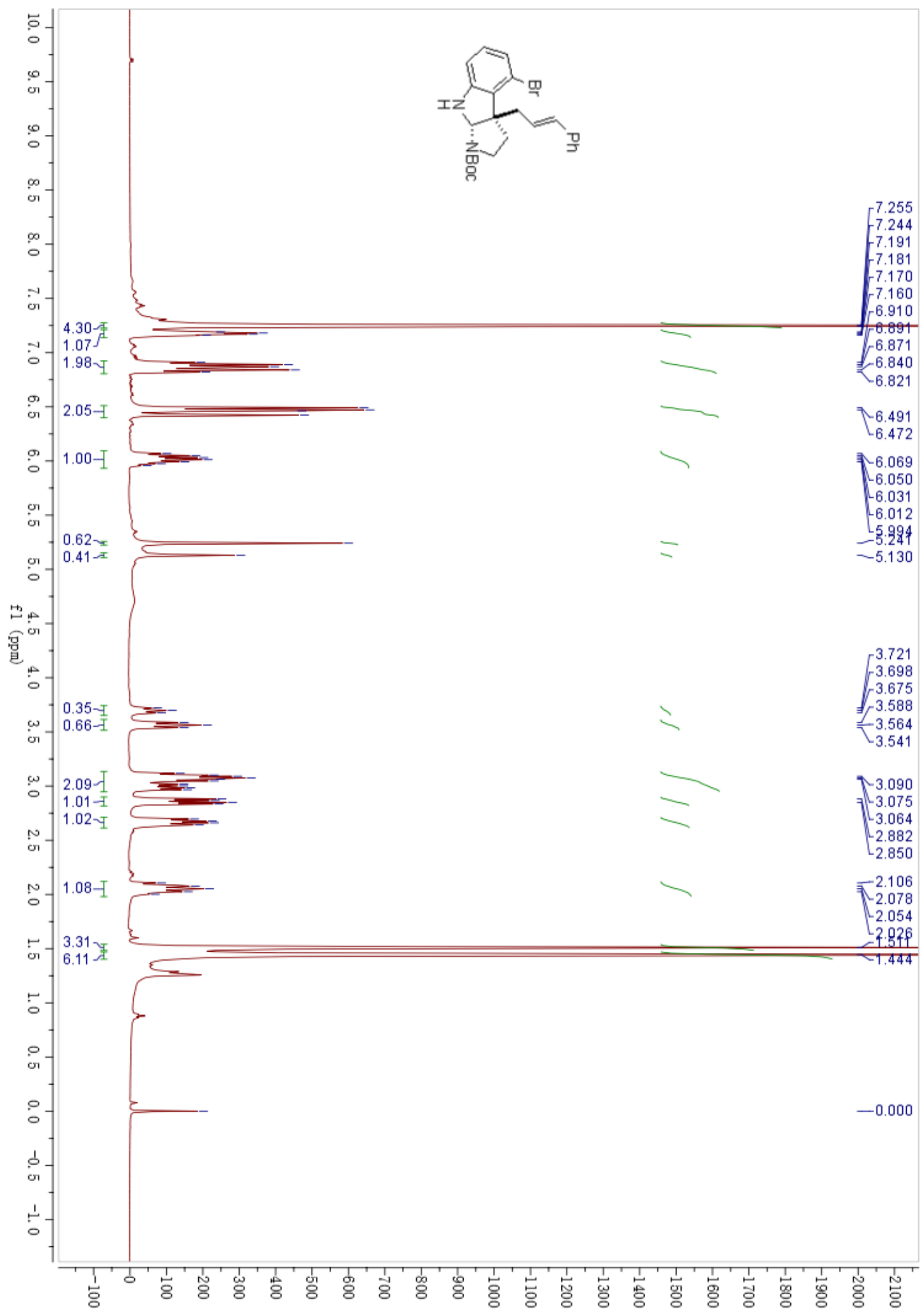


${ }^{13} \mathrm{C}$ NMR spectra of $\mathbf{3 I}$

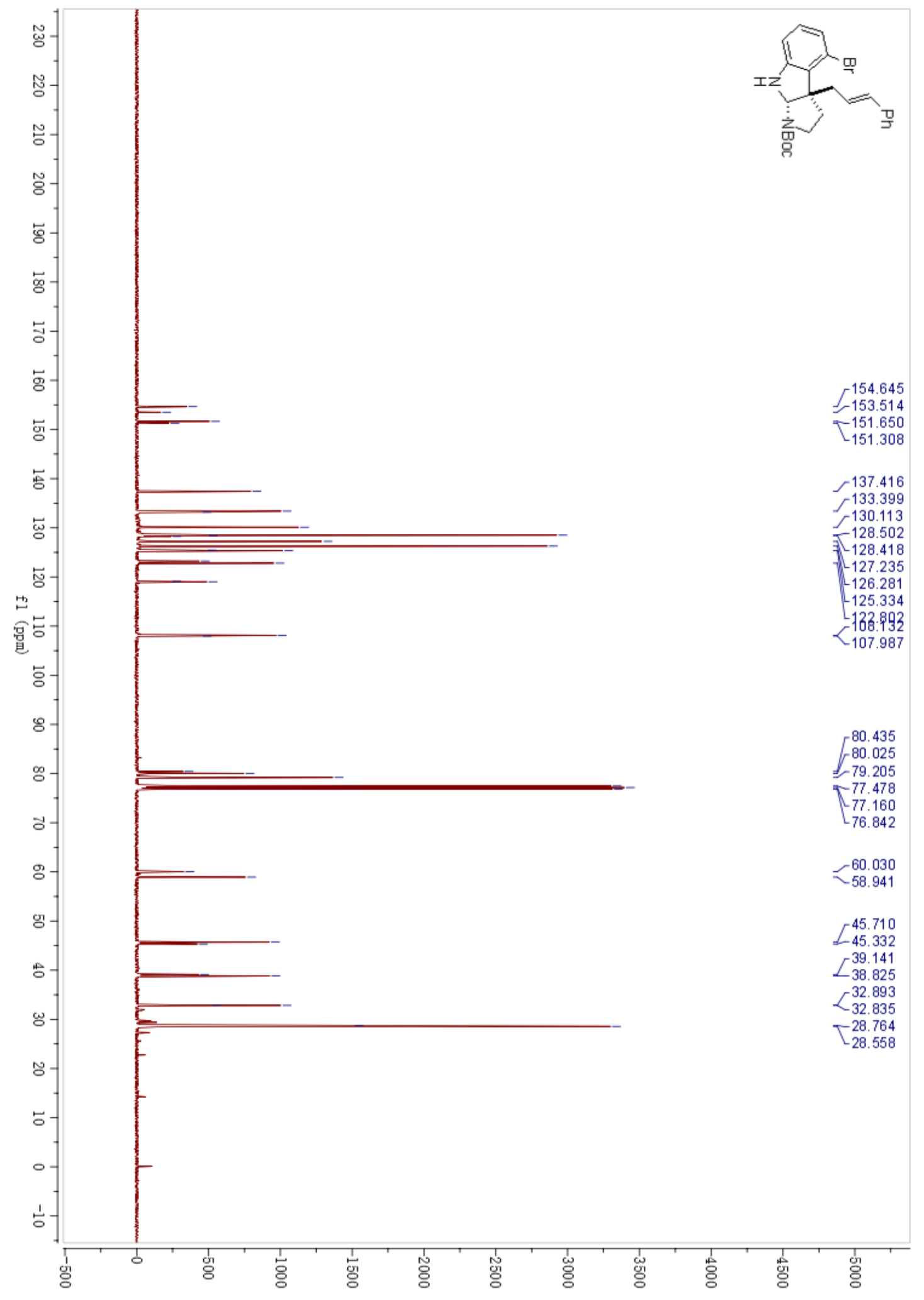


${ }^{1} \mathrm{H}$ NMR spectra of $\mathbf{3 m}$

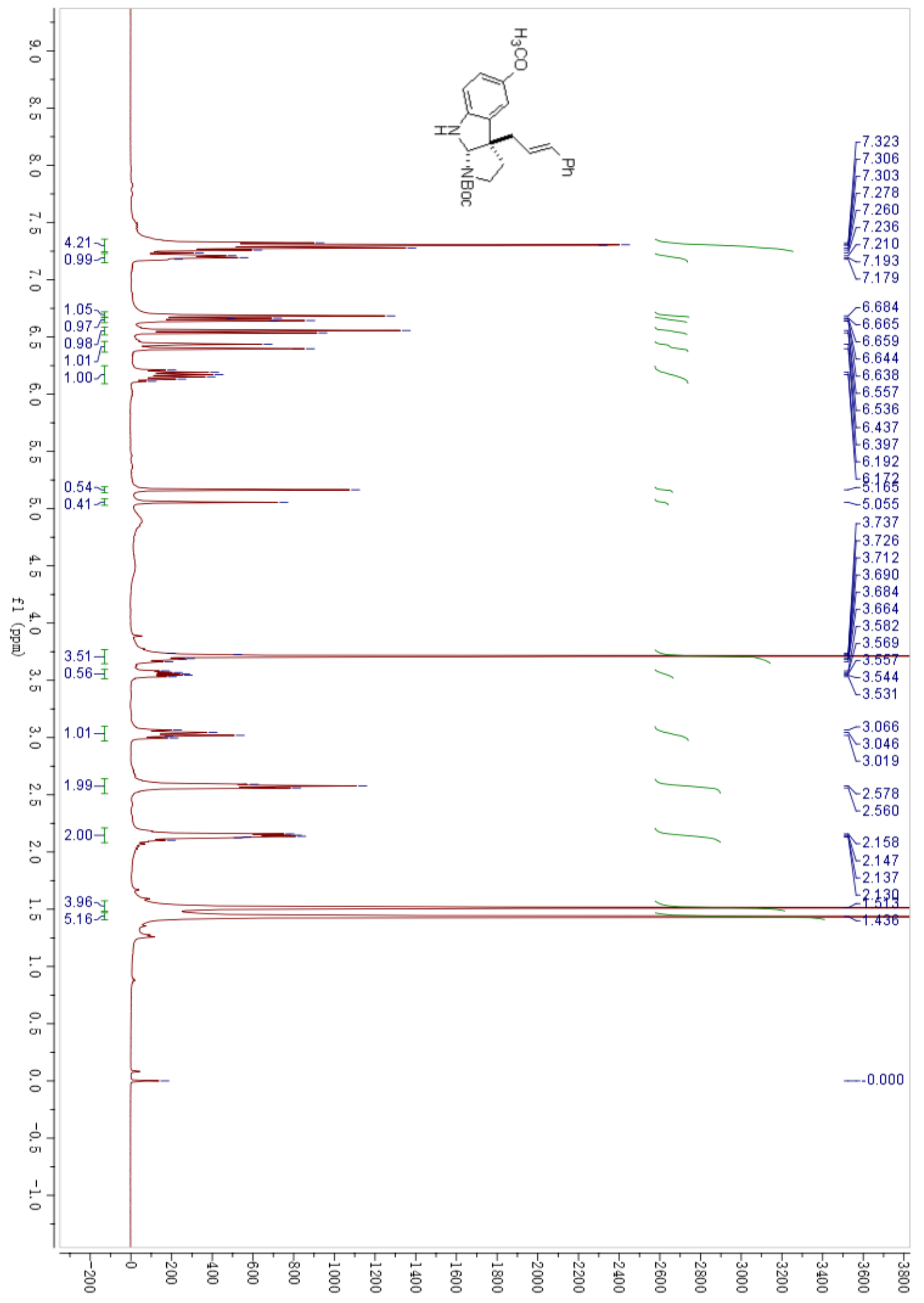


${ }^{1} \mathrm{H}$ NMR spectra of $\mathbf{3 n}$

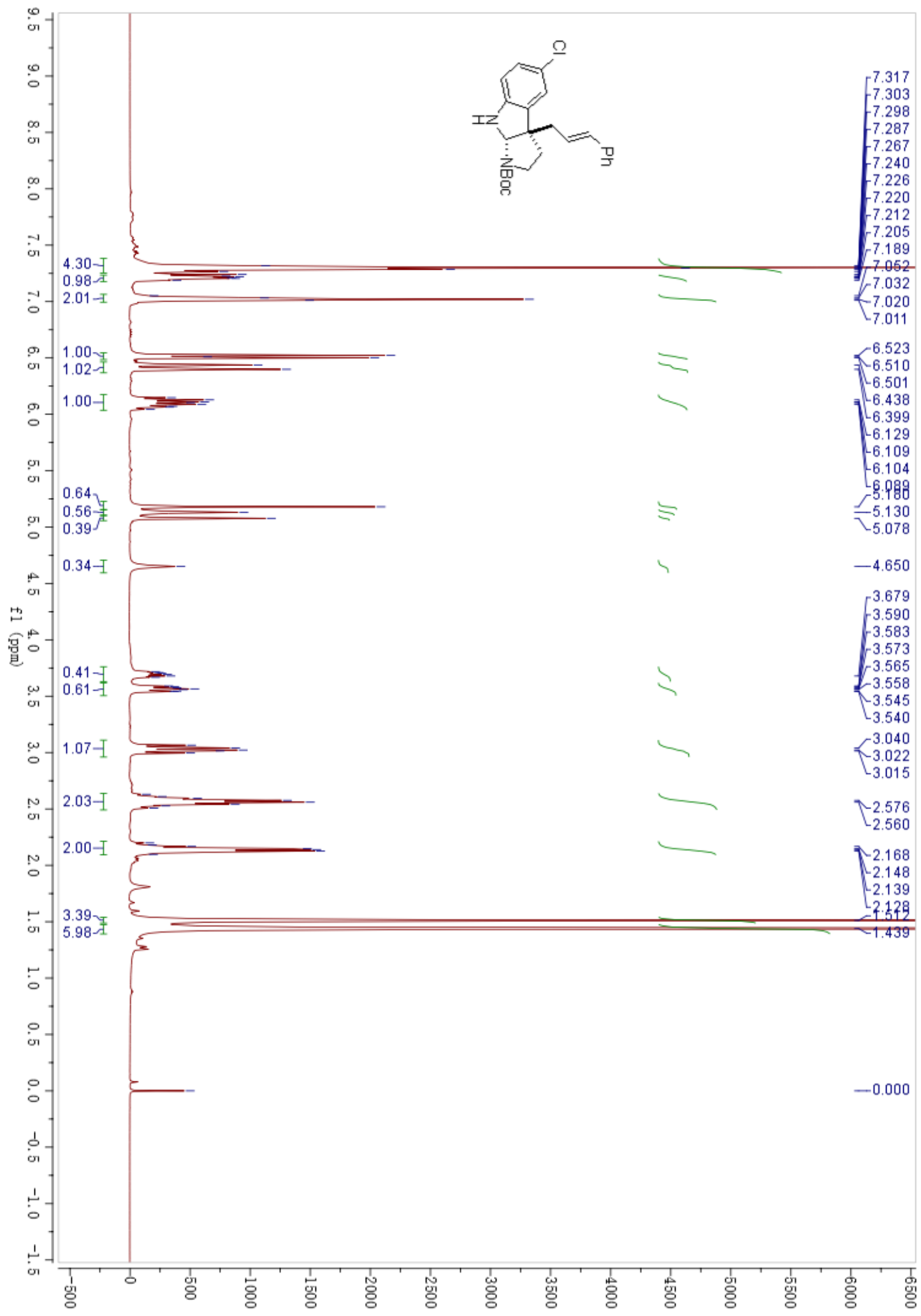


${ }^{13} \mathrm{C}$ NMR spectra of $\mathbf{3 n}$

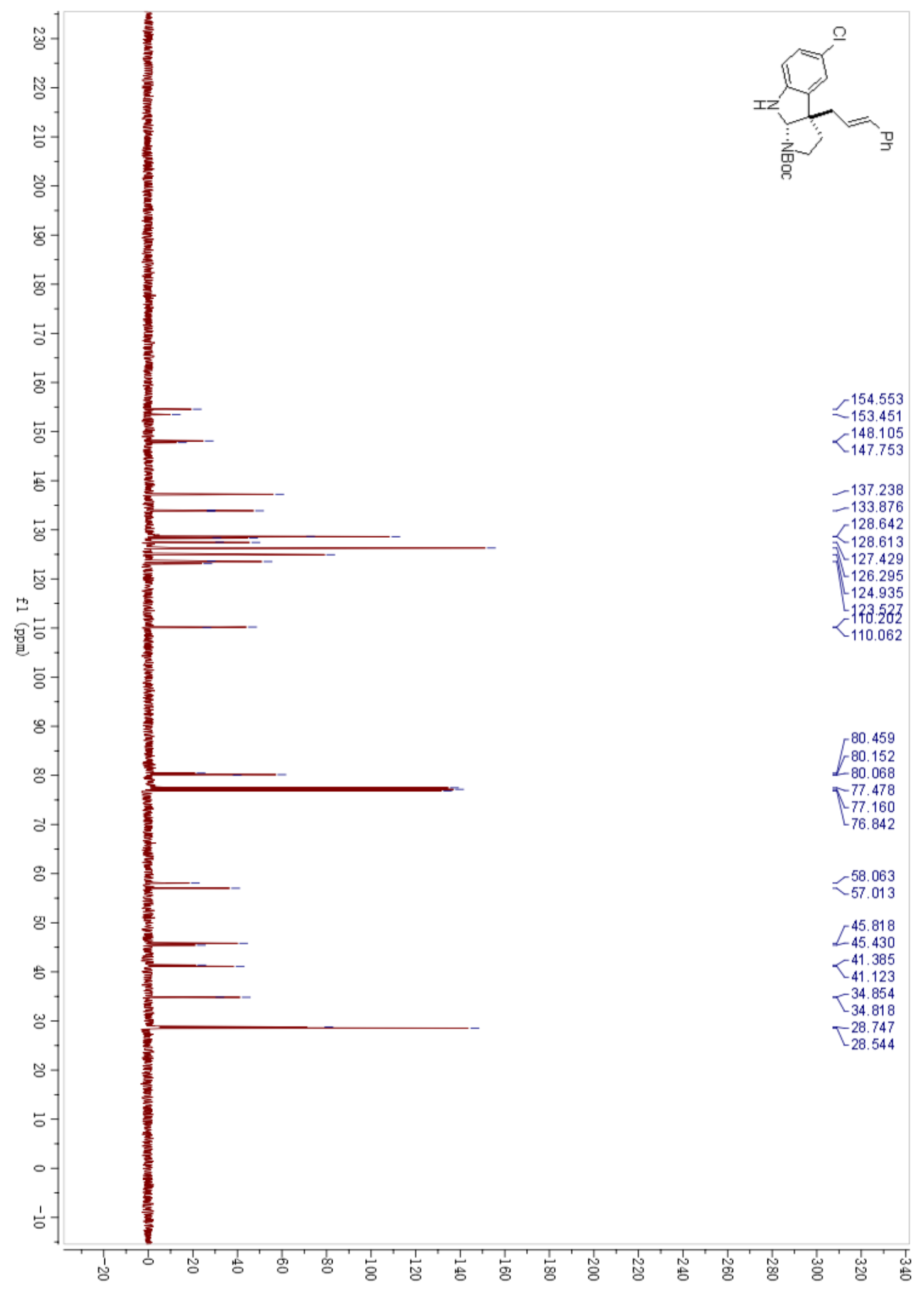


${ }^{1} \mathrm{H}$ NMR spectra of $\mathbf{3 o}$

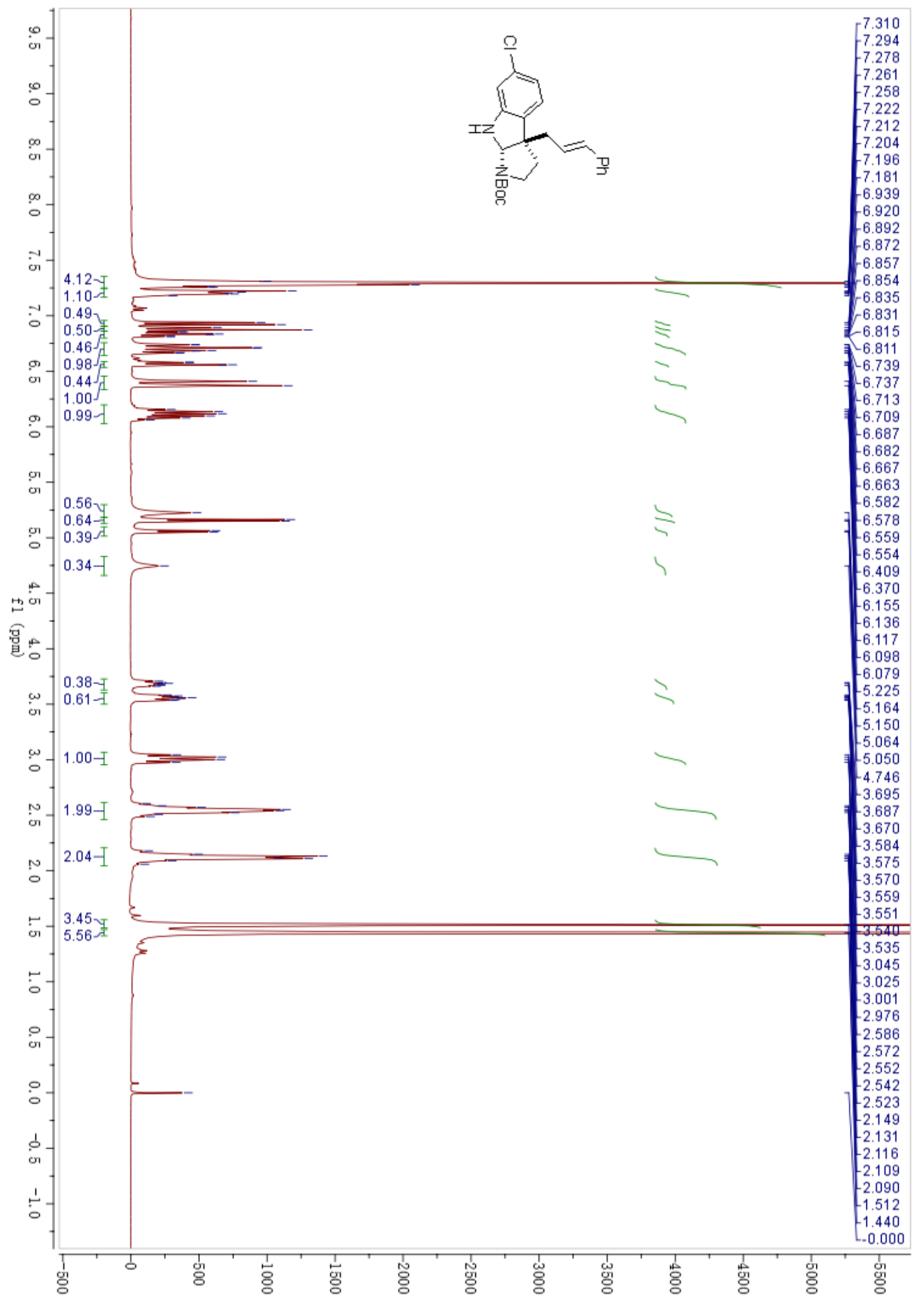


${ }^{13} \mathrm{C}$ NMR spectra of $\mathbf{3 o}$

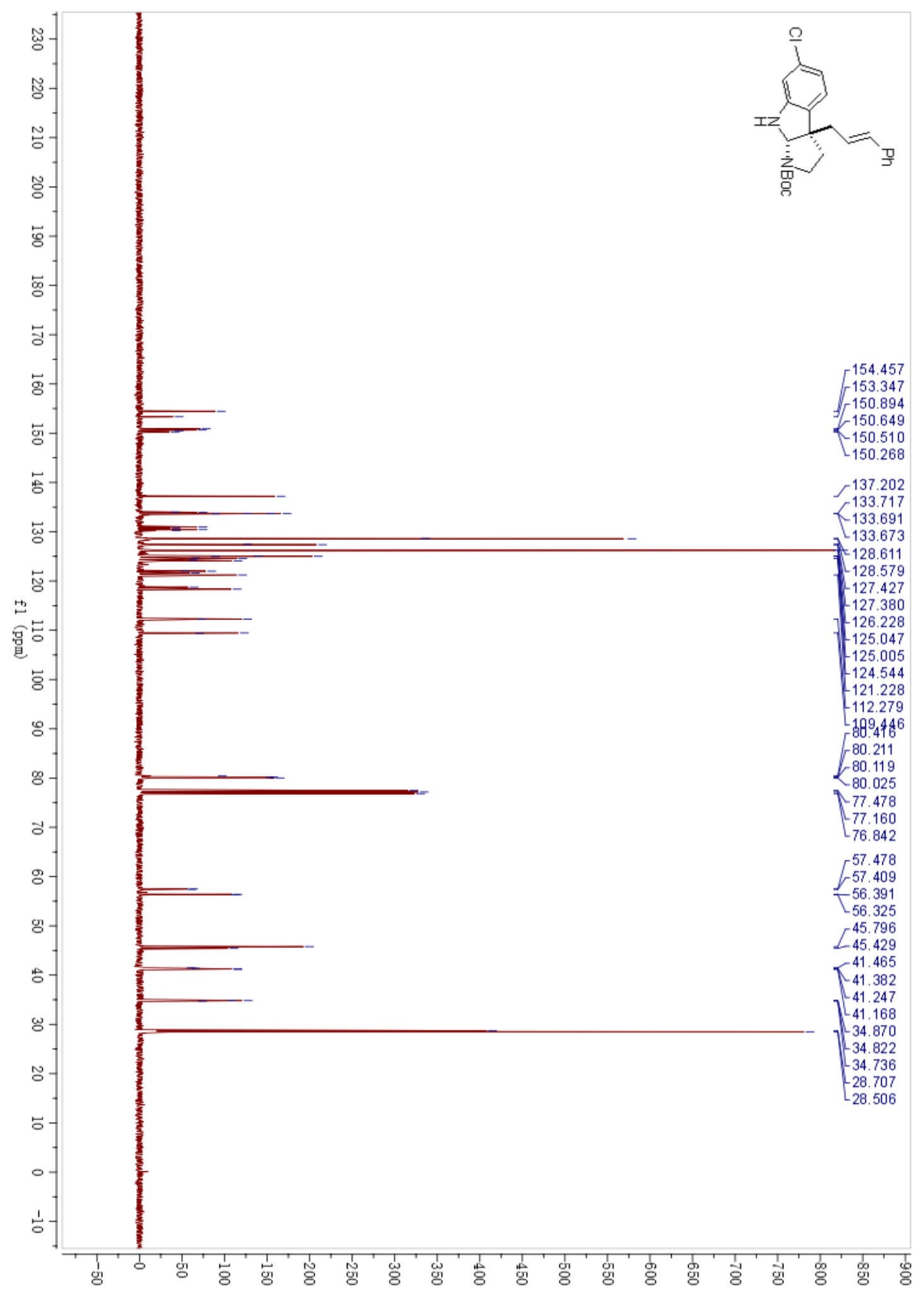


${ }^{1} \mathrm{H}$ NMR spectra of $\mathbf{3 0}{ }^{\prime}$

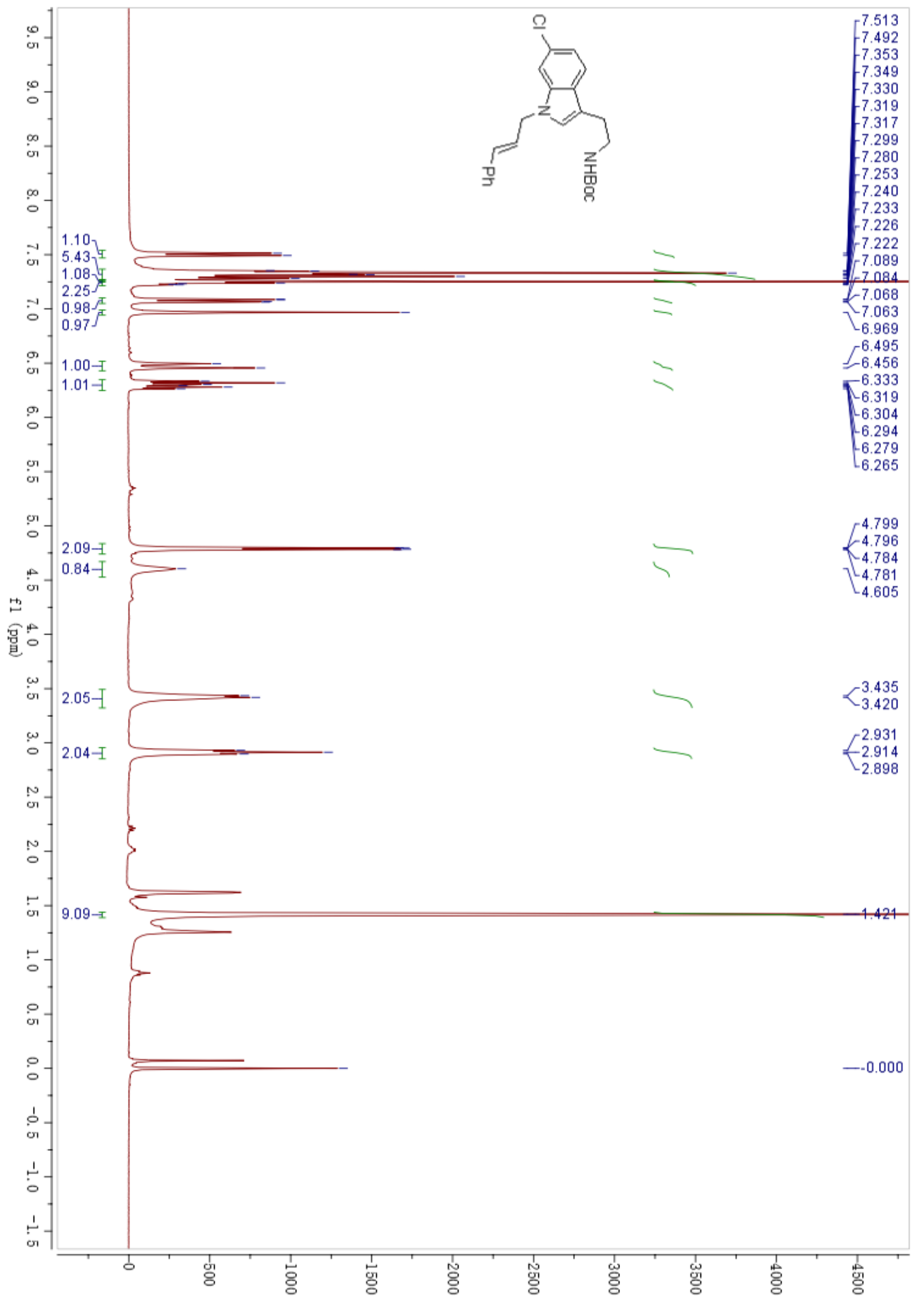


${ }^{13} \mathrm{C}$ NMR spectra of $\mathbf{3 0}{ }^{\prime}$

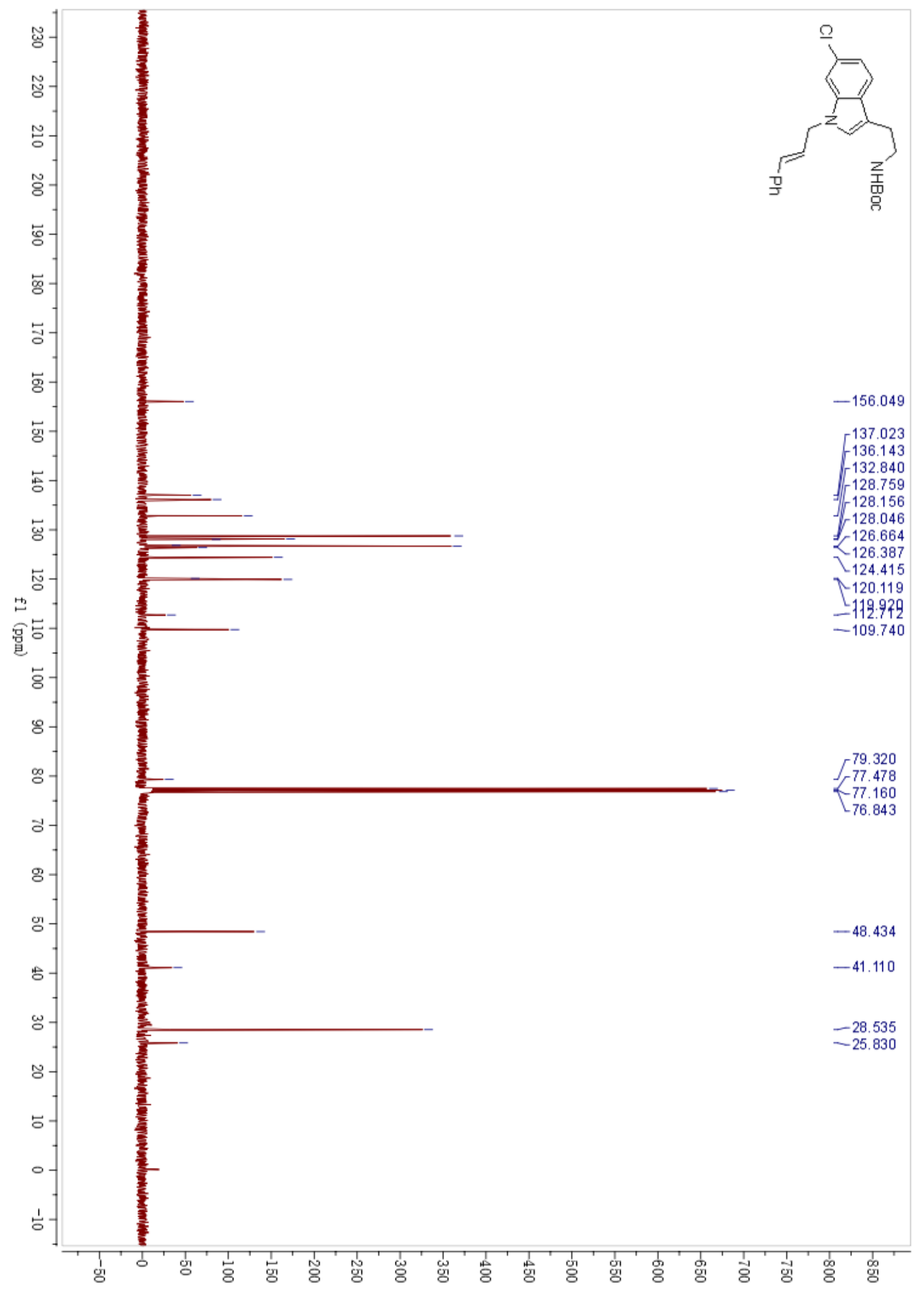


${ }^{1} \mathrm{H}$ NMR spectra of $\mathbf{3 p}$

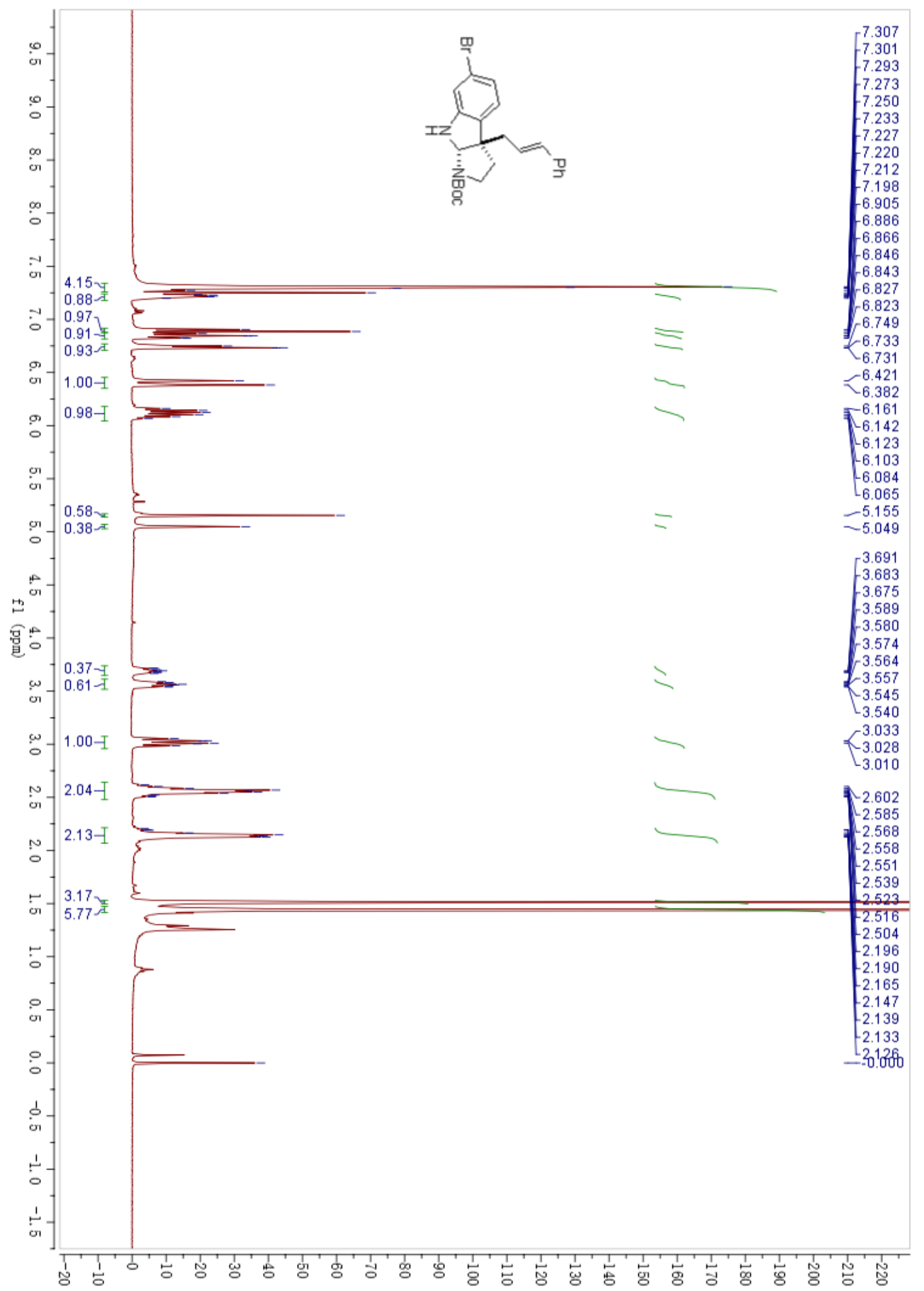


${ }^{13} \mathrm{C}$ NMR spectra of $\mathbf{3 p}$

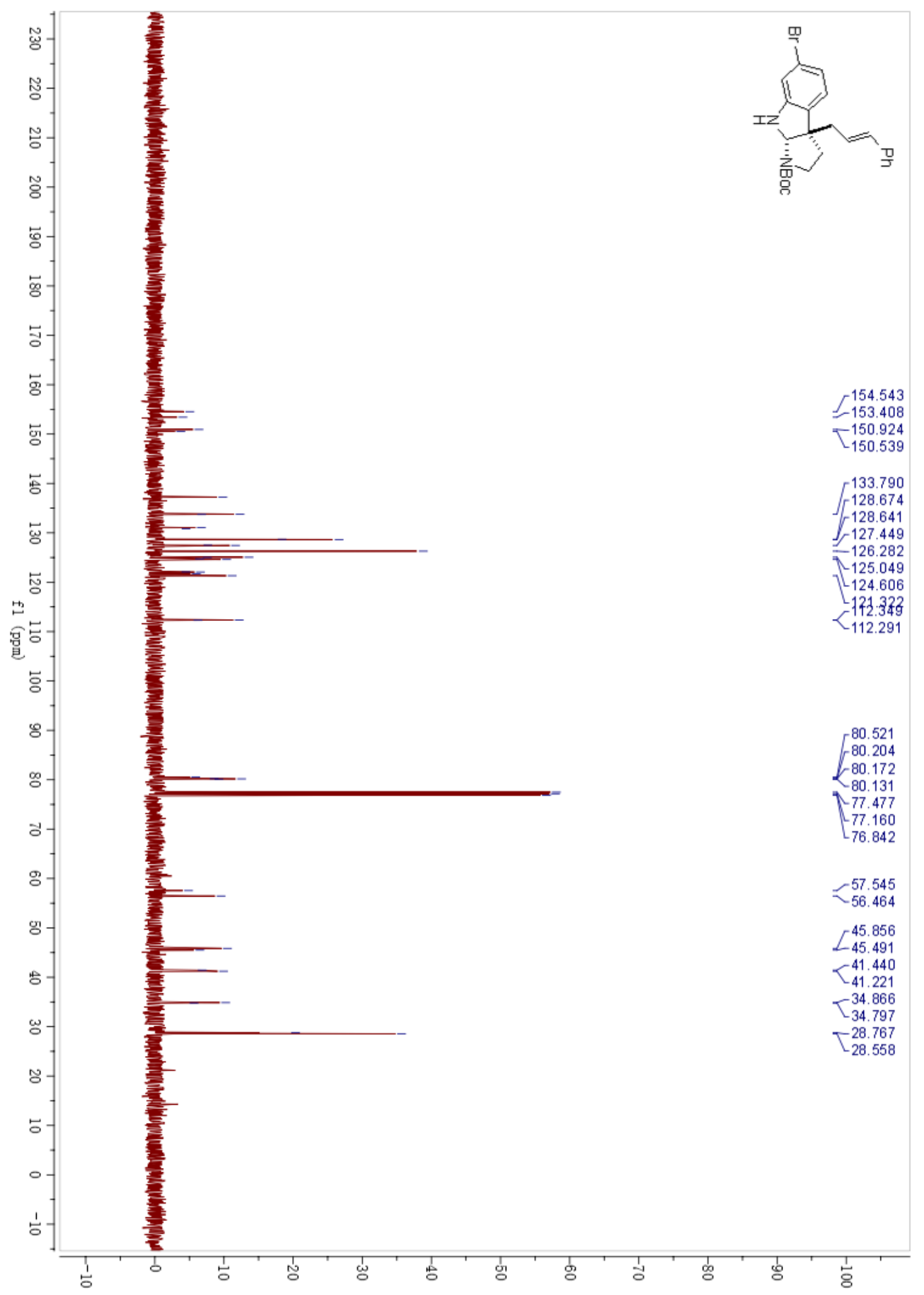


${ }^{1} \mathrm{H}$ NMR spectra of $\mathbf{3} \mathbf{q}_{\text {(major) }}$

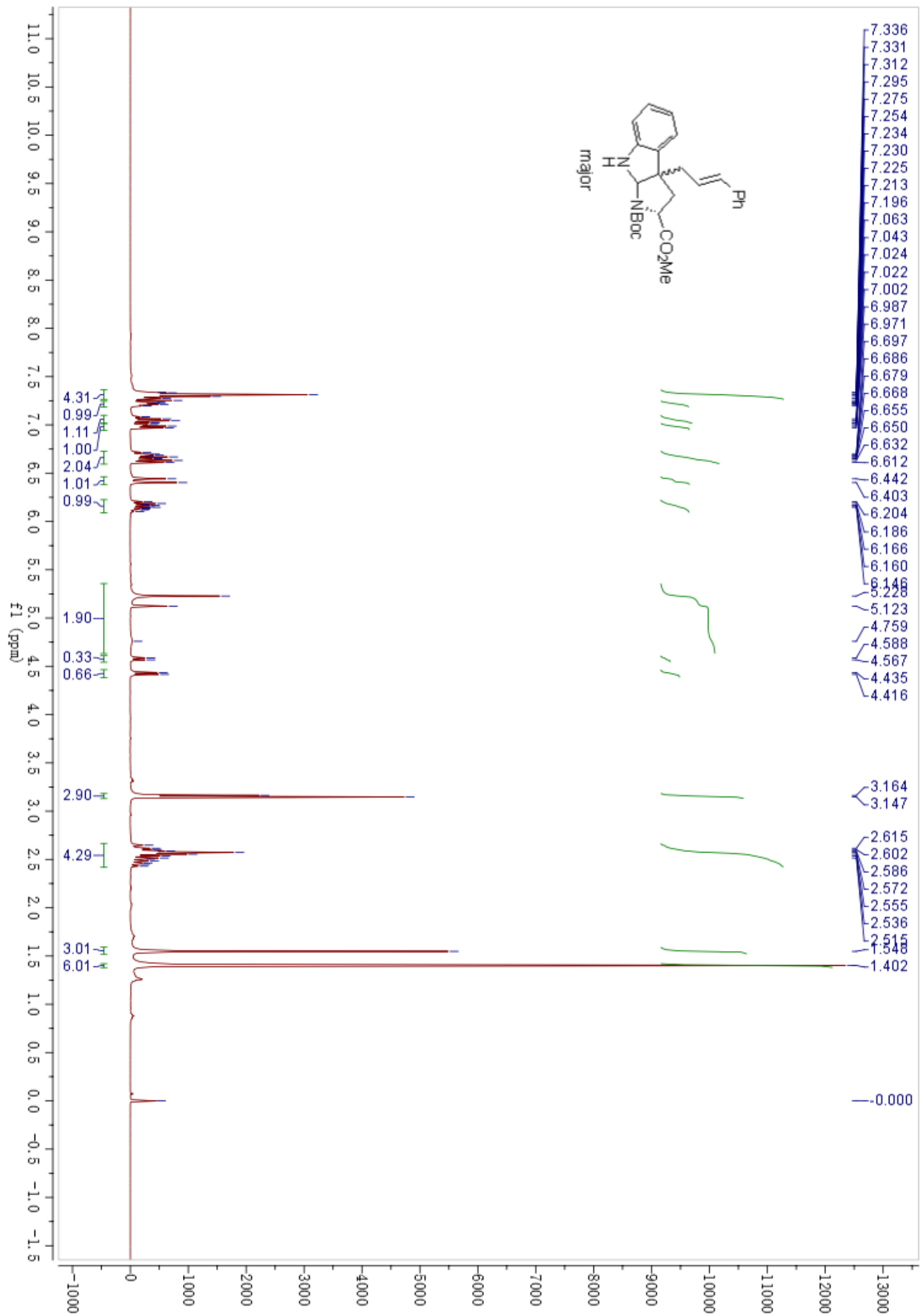


${ }^{13} \mathrm{C}$ NMR spectra of $\mathbf{3} \mathbf{q}_{(\text {major })}$

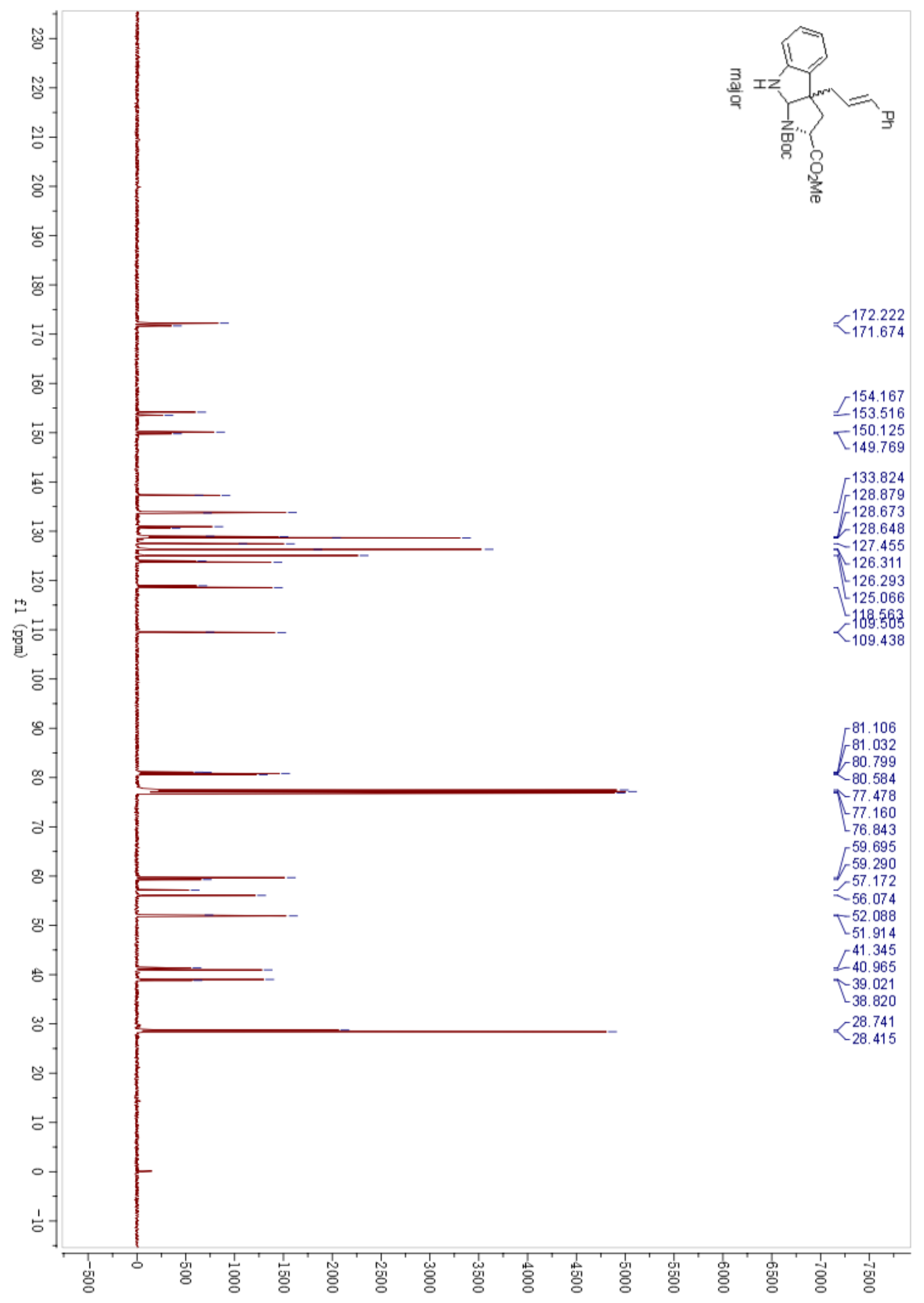


${ }^{1} \mathrm{H}$ NMR spectra of $\mathbf{3} \mathbf{q}_{(\text {minor })}$

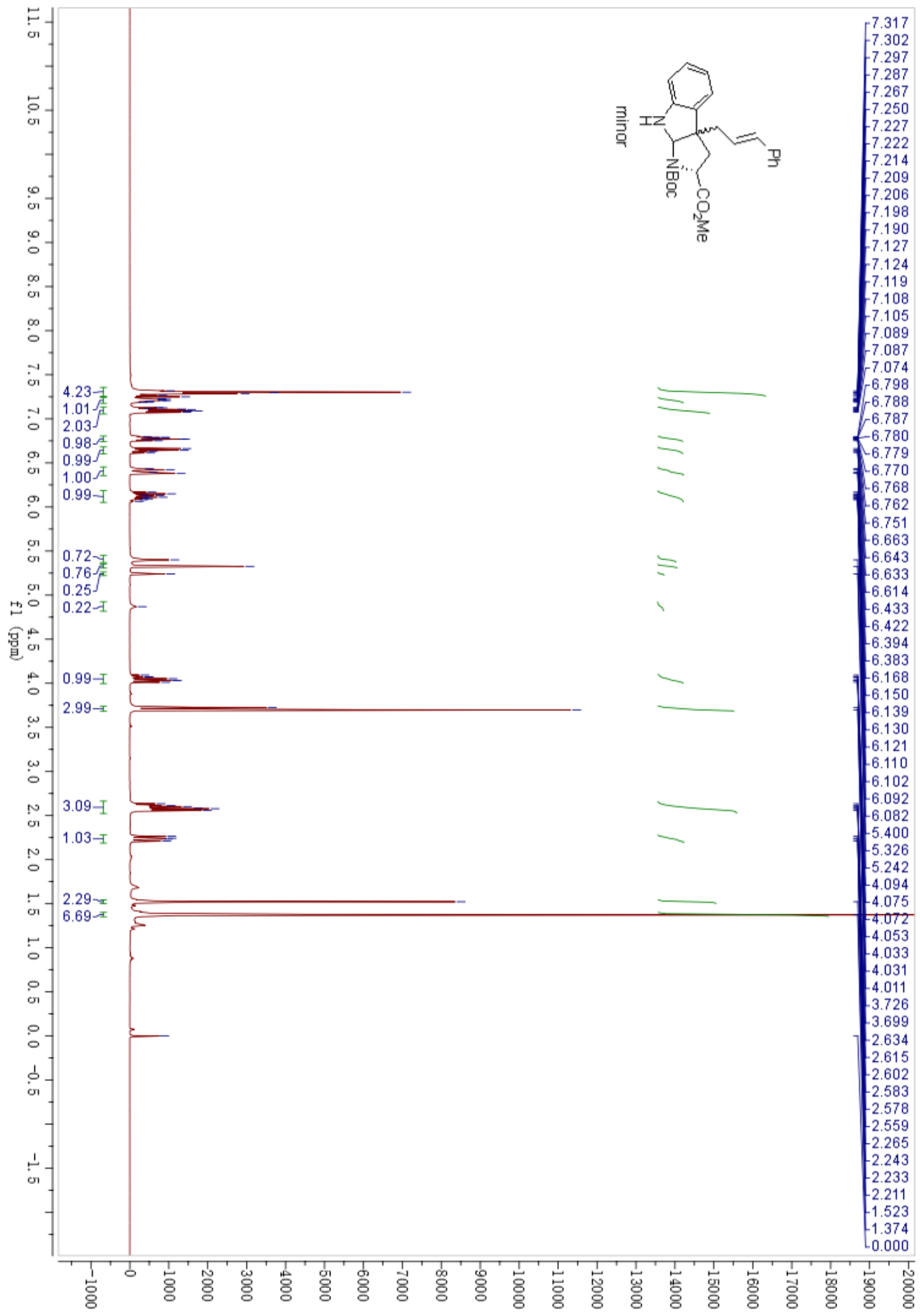


${ }^{13} \mathrm{C}$ NMR spectra of $\mathbf{3} \mathbf{q}_{(\text {minor })}$

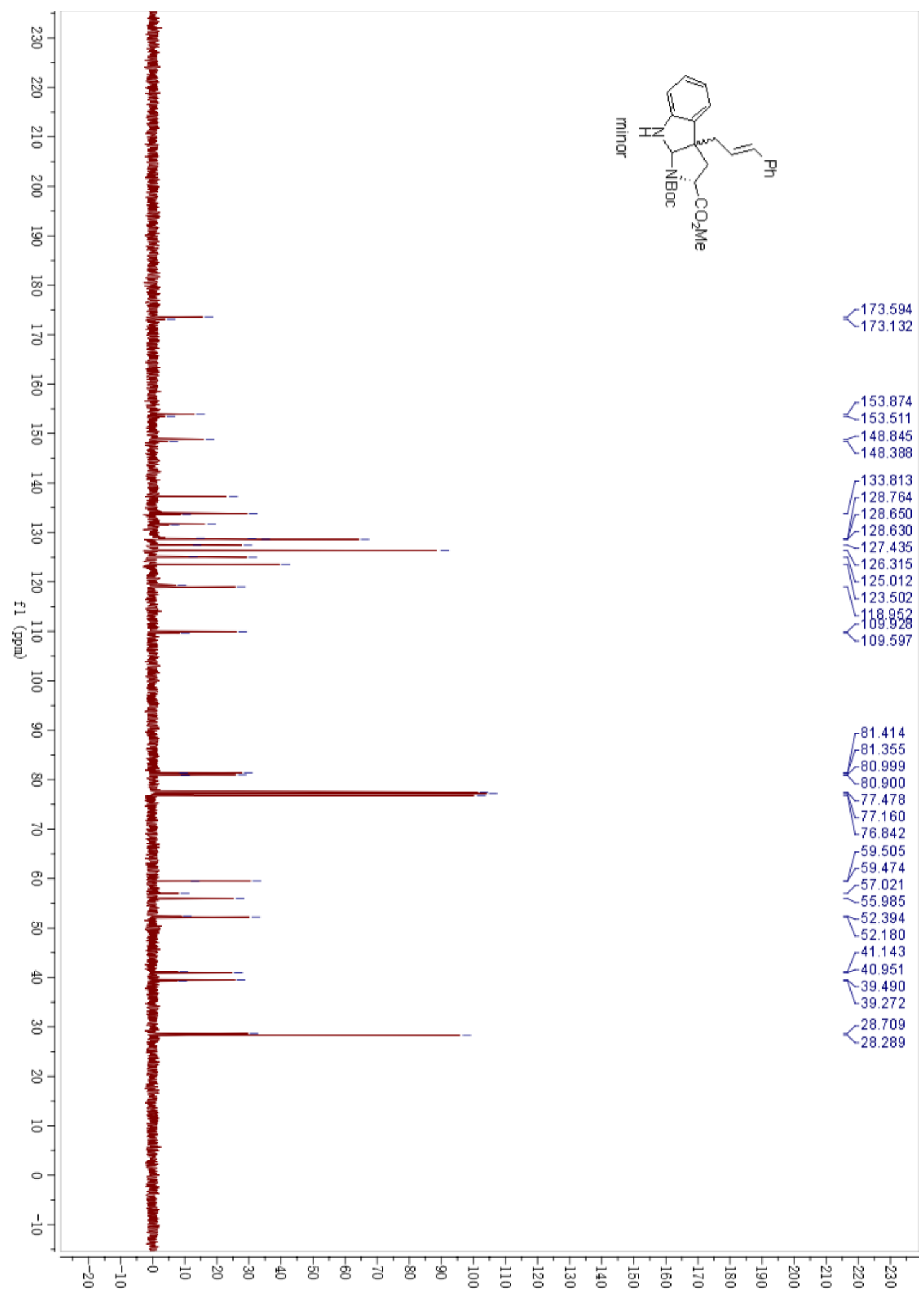


${ }^{1} \mathrm{H}$ NMR spectra of $\mathbf{3} \mathbf{r}_{\text {(major) }}$

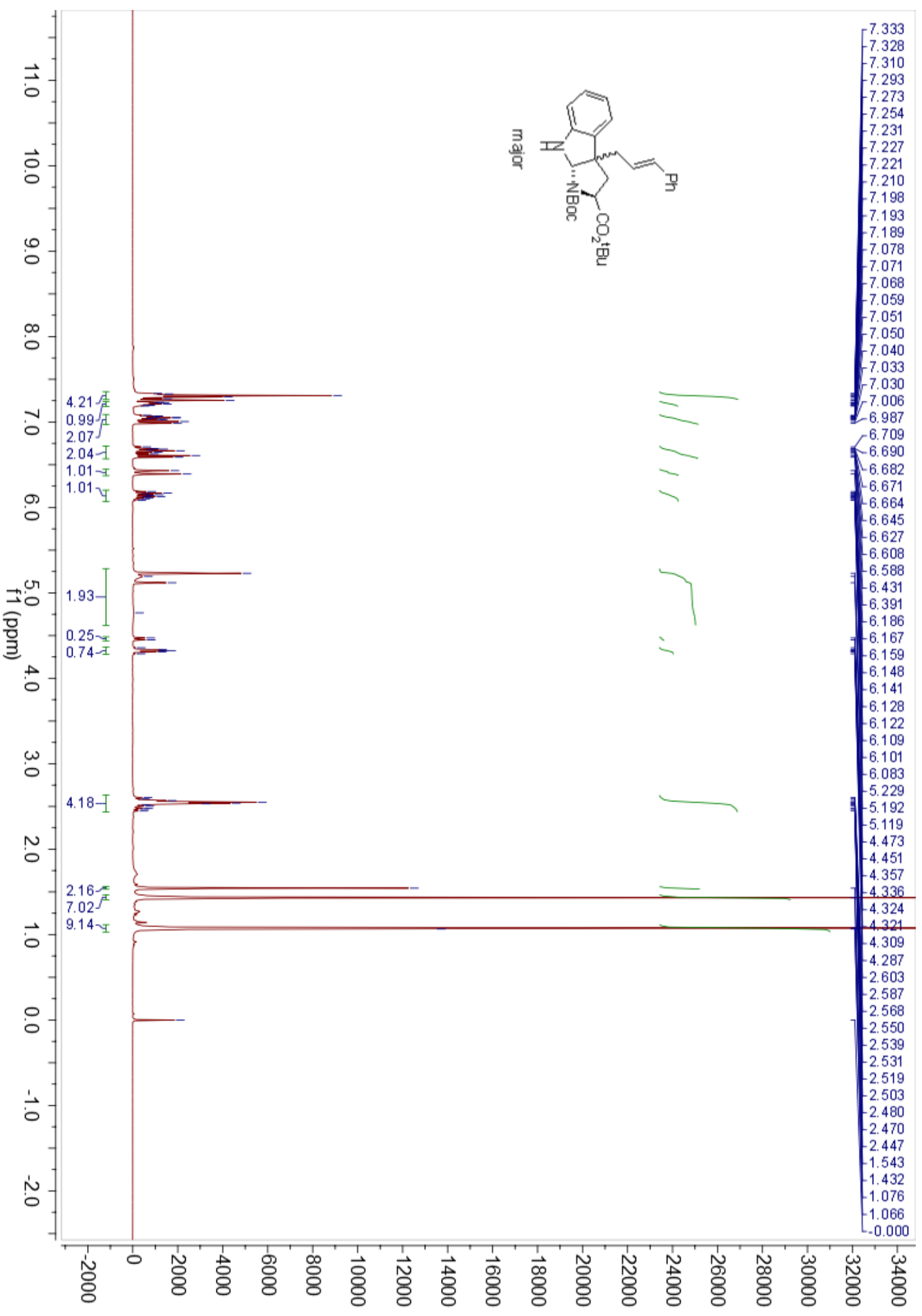


${ }^{13} \mathrm{C}$ NMR spectra of $\mathbf{3} \mathbf{r}_{\text {(major) }}$

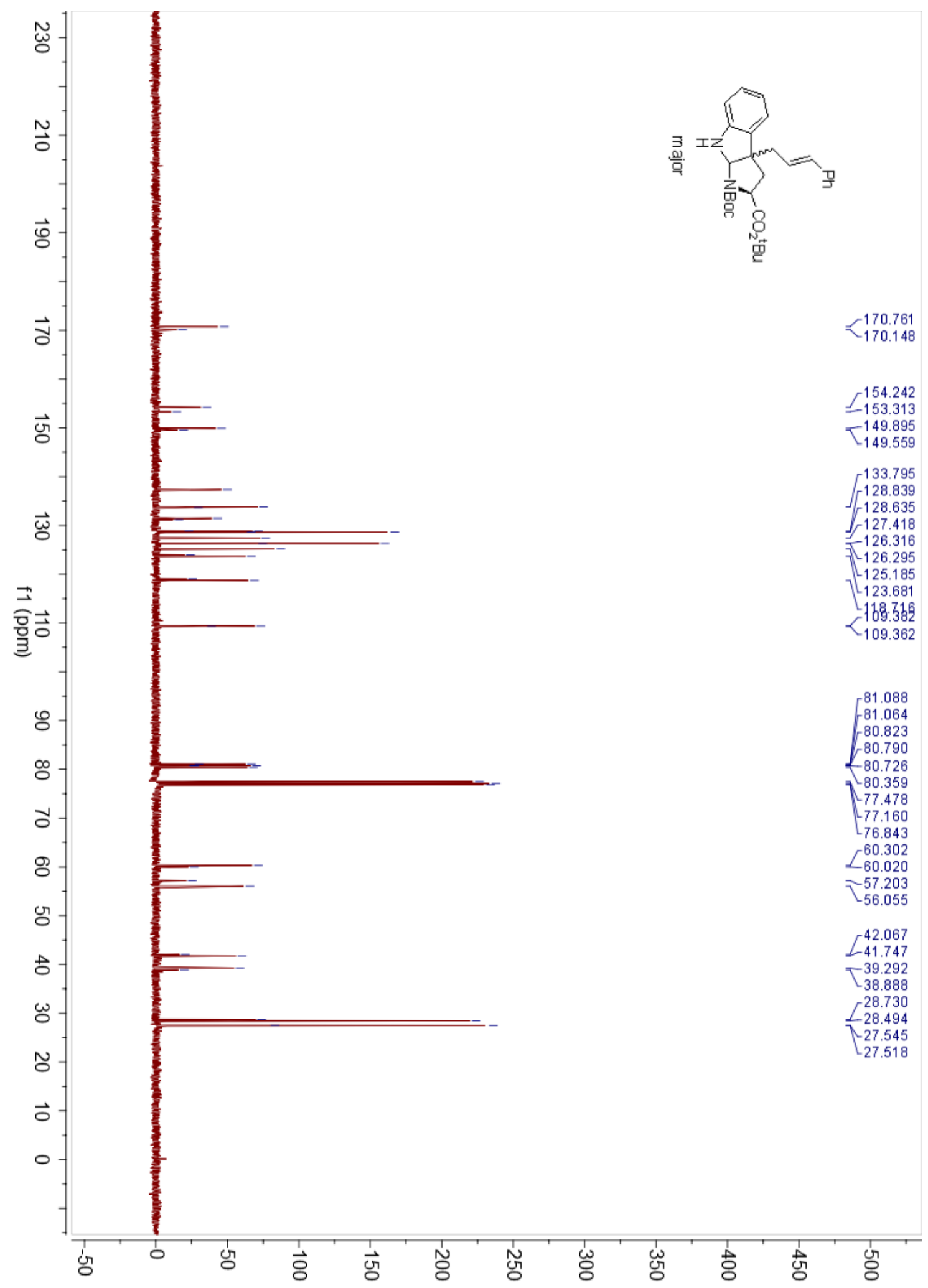


${ }^{1} \mathrm{H}$ NMR spectra of $\mathbf{3} \mathbf{r}_{(\text {minor) }}$

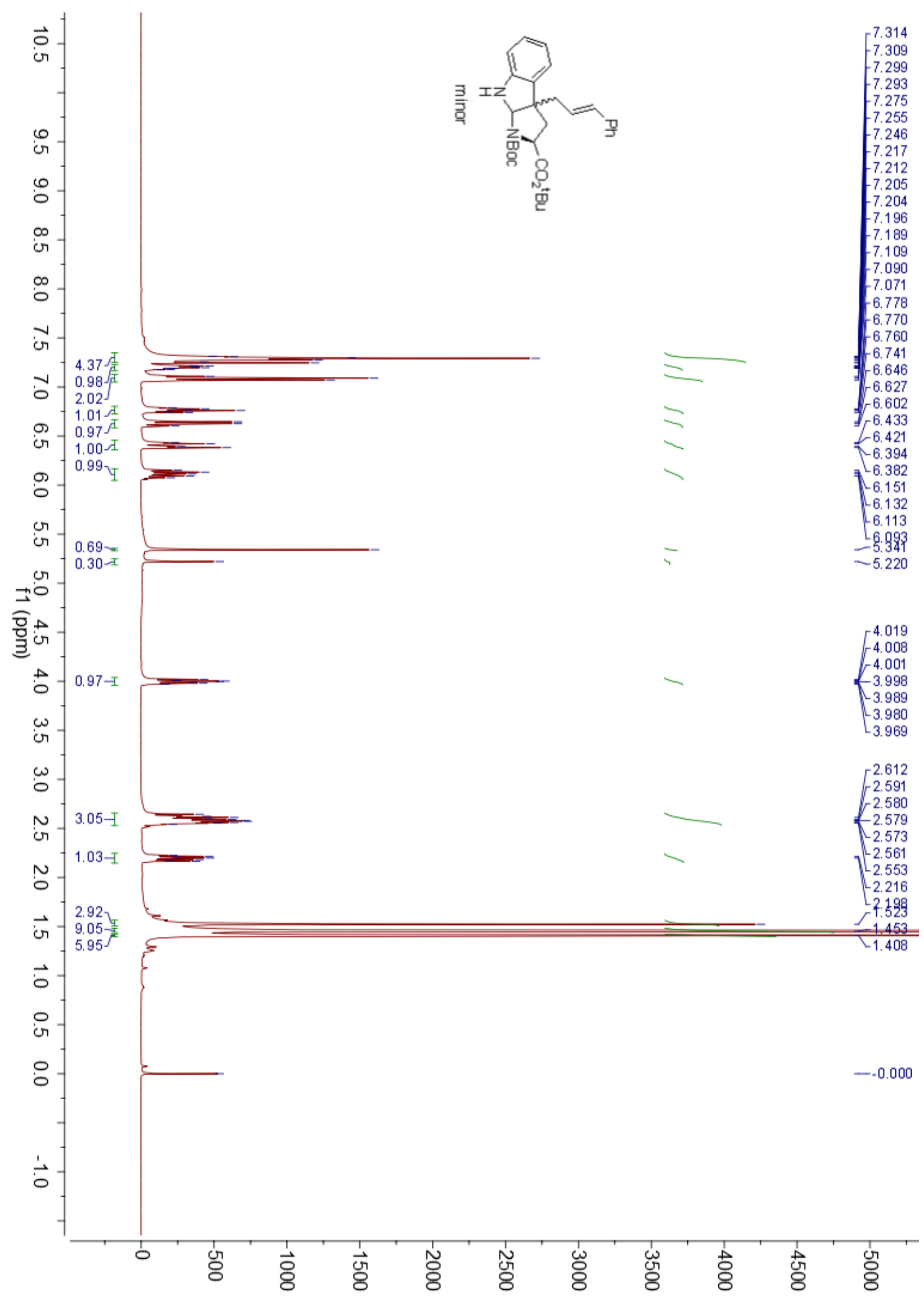


${ }^{13} \mathrm{C}$ NMR spectra of $\mathbf{3} \mathbf{r}_{(\text {minor })}$

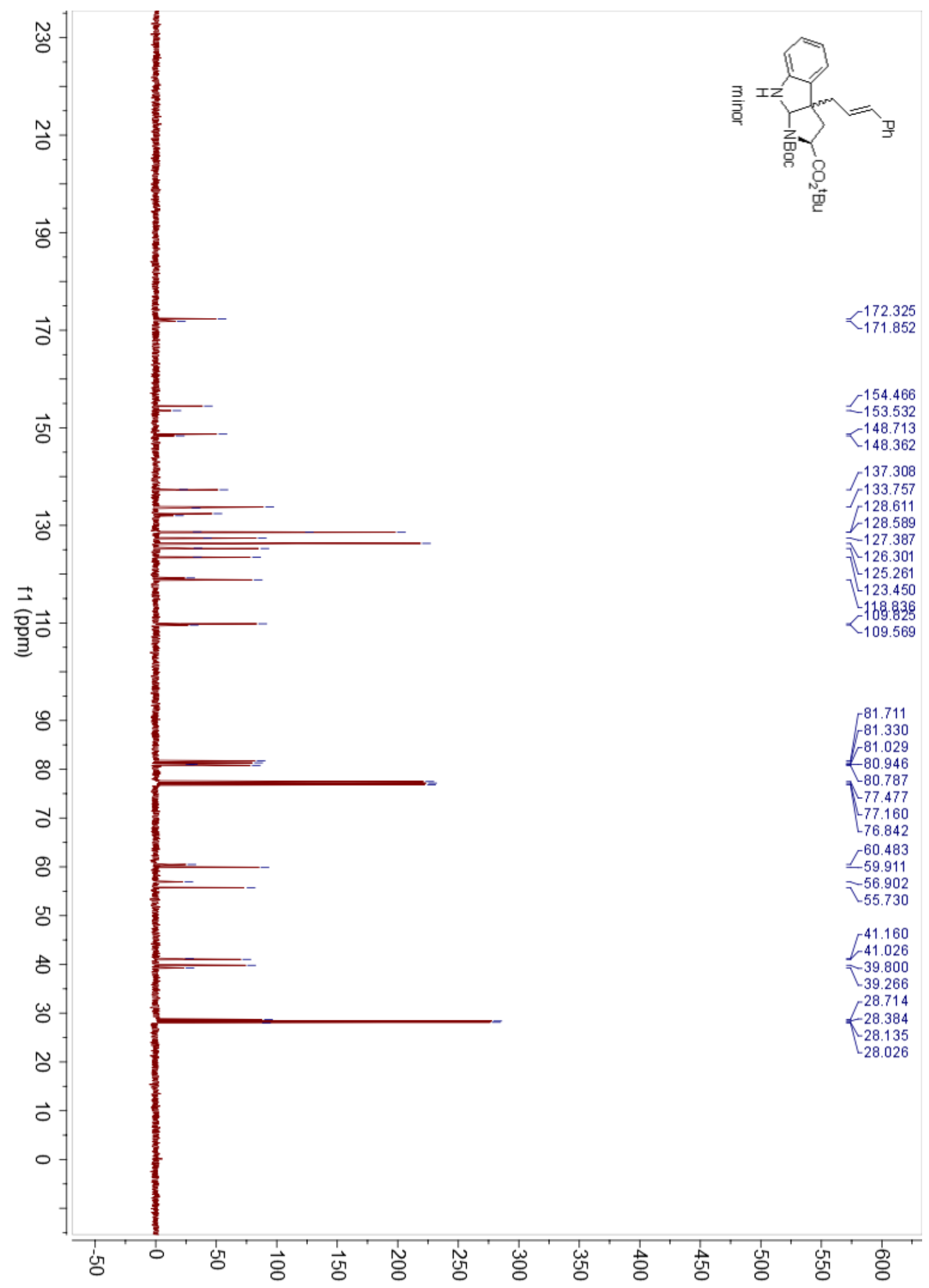


${ }^{1} \mathrm{H}$ NMR spectra of $\mathbf{3 s}$

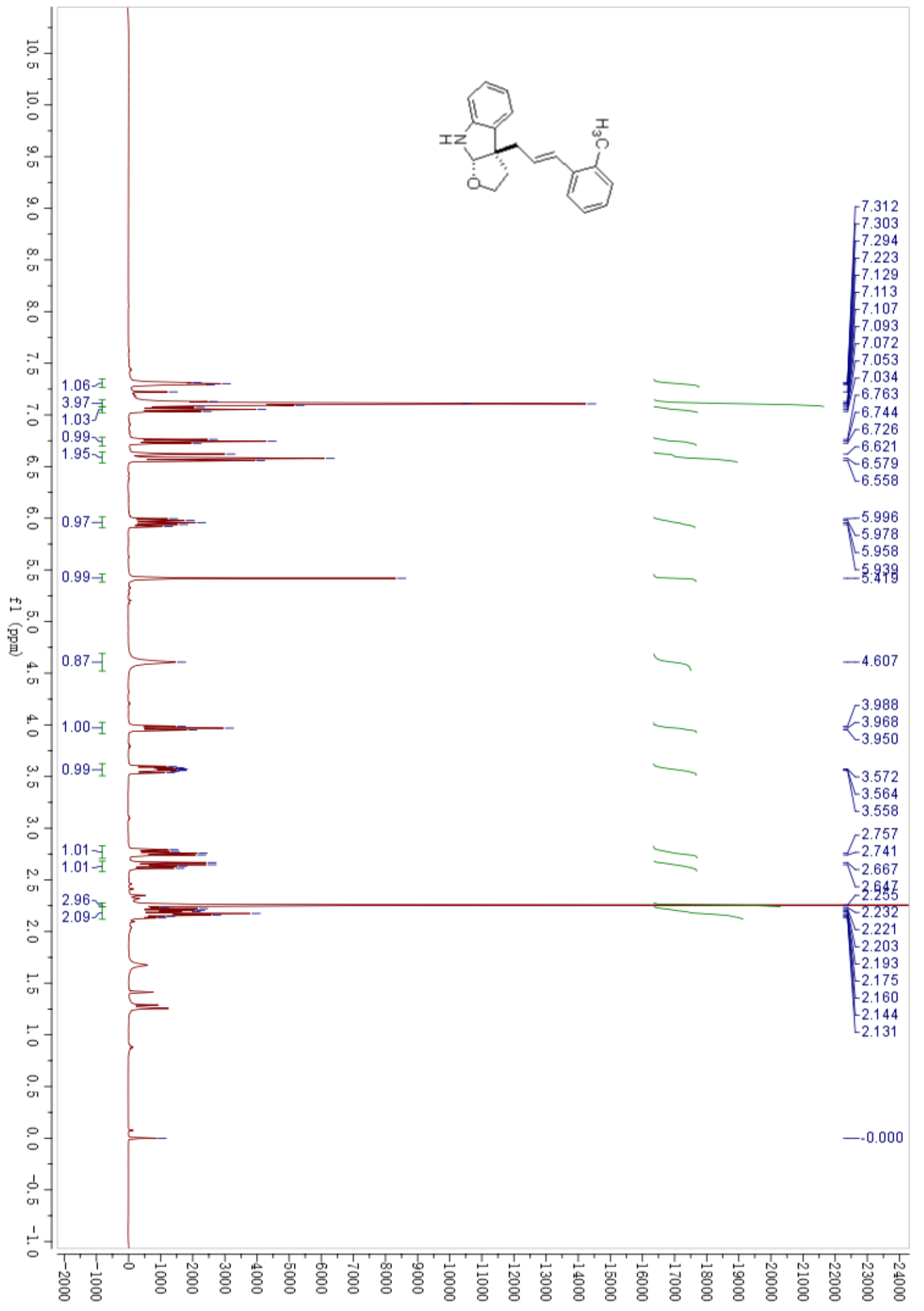


${ }^{13} \mathrm{C}$ NMR spectra of $\mathbf{3 s}$

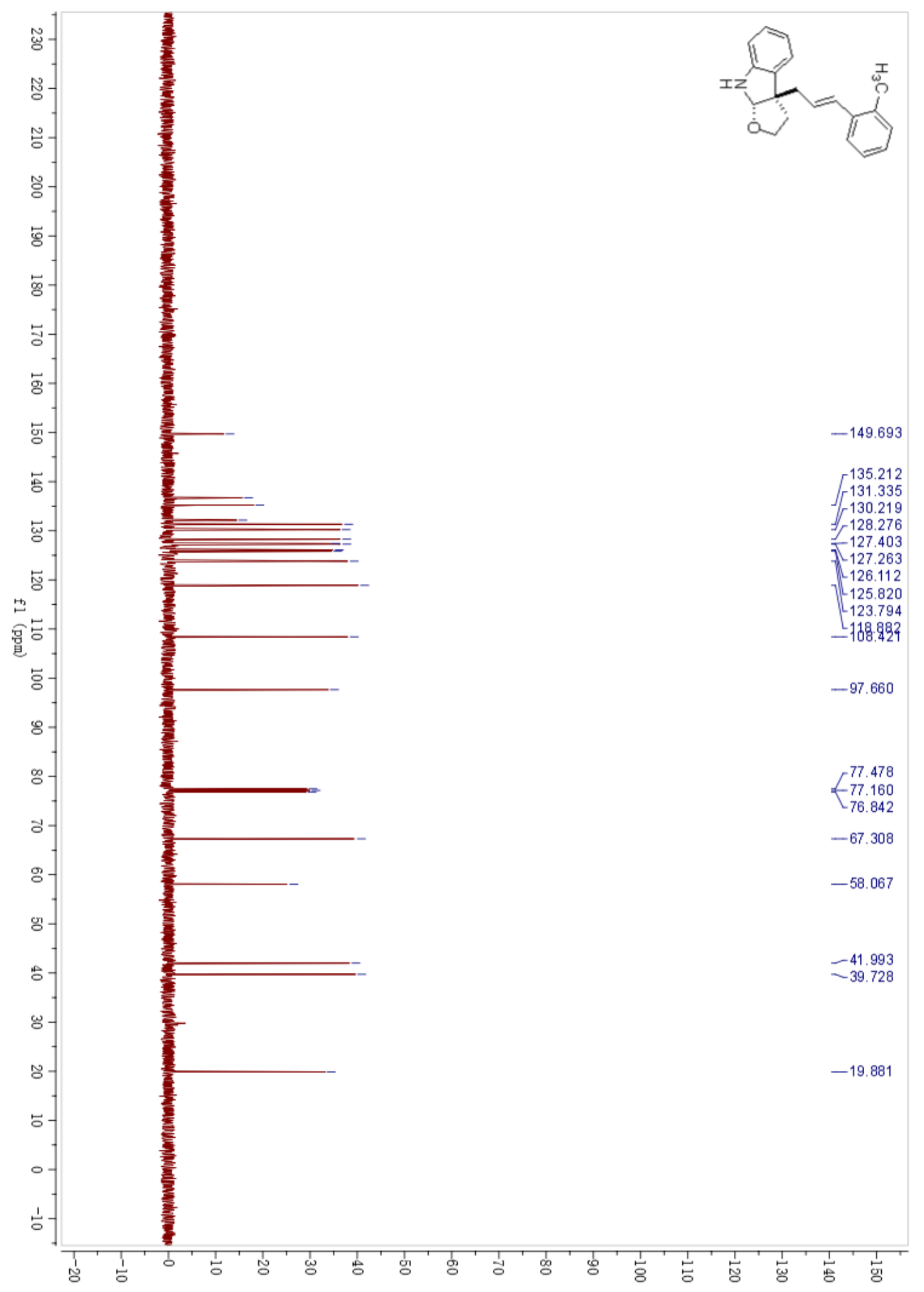


${ }^{1} \mathrm{H}$ NMR spectra of $\mathbf{3 t}$

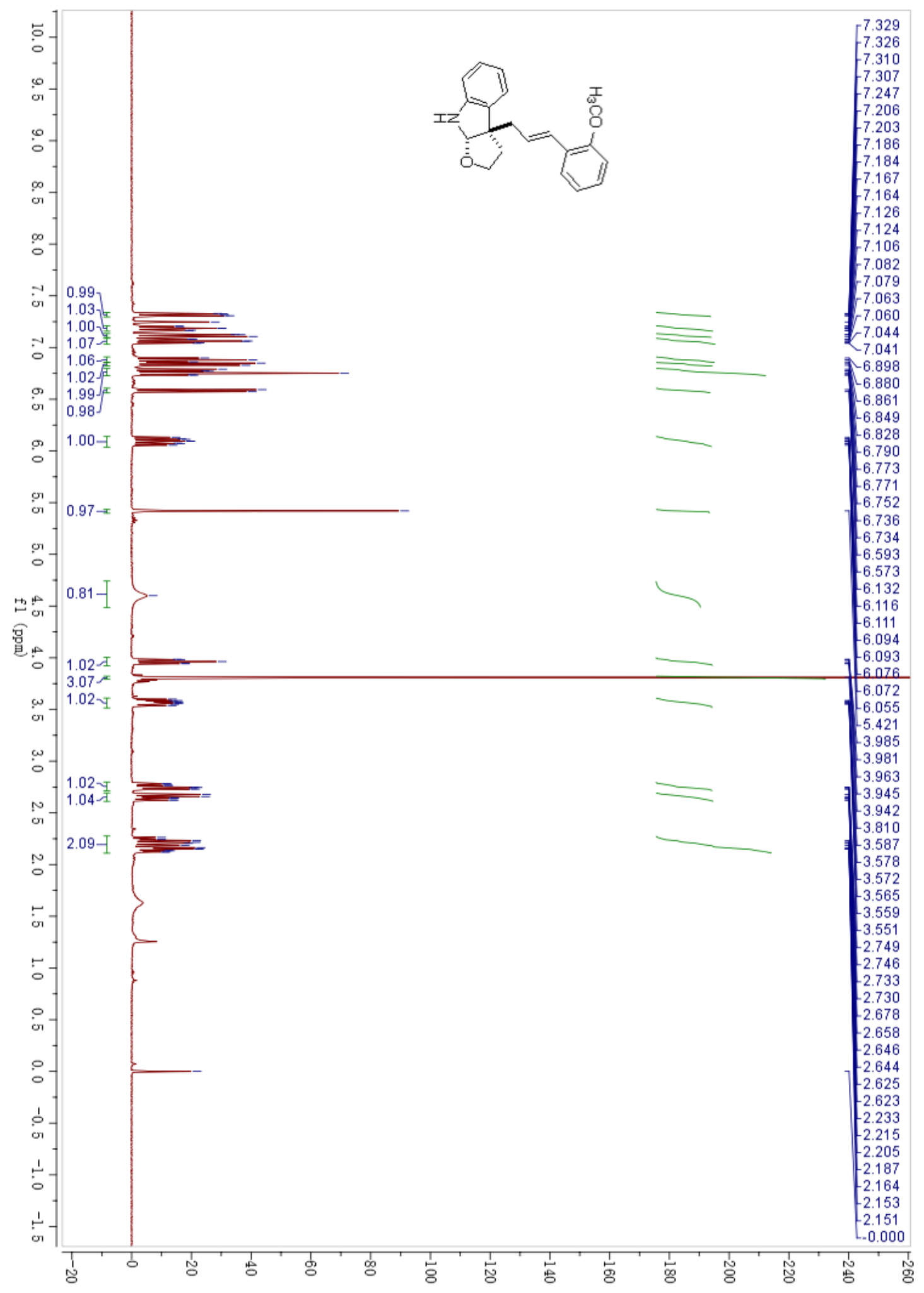


${ }^{13} \mathrm{C}$ NMR spectra of $\mathbf{3 t}$

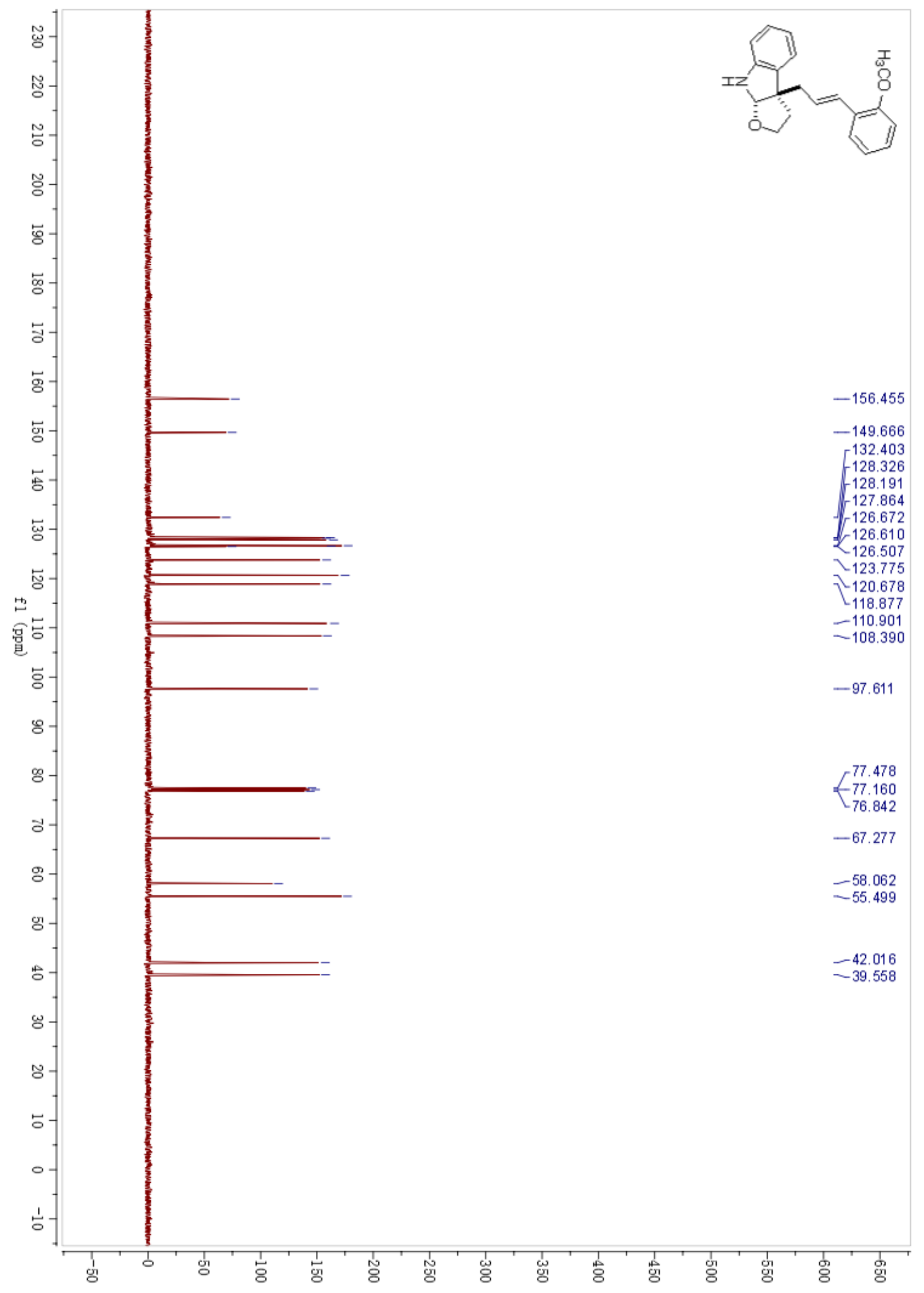


${ }^{1}$ H NMR spectra of $\mathbf{3 u}$

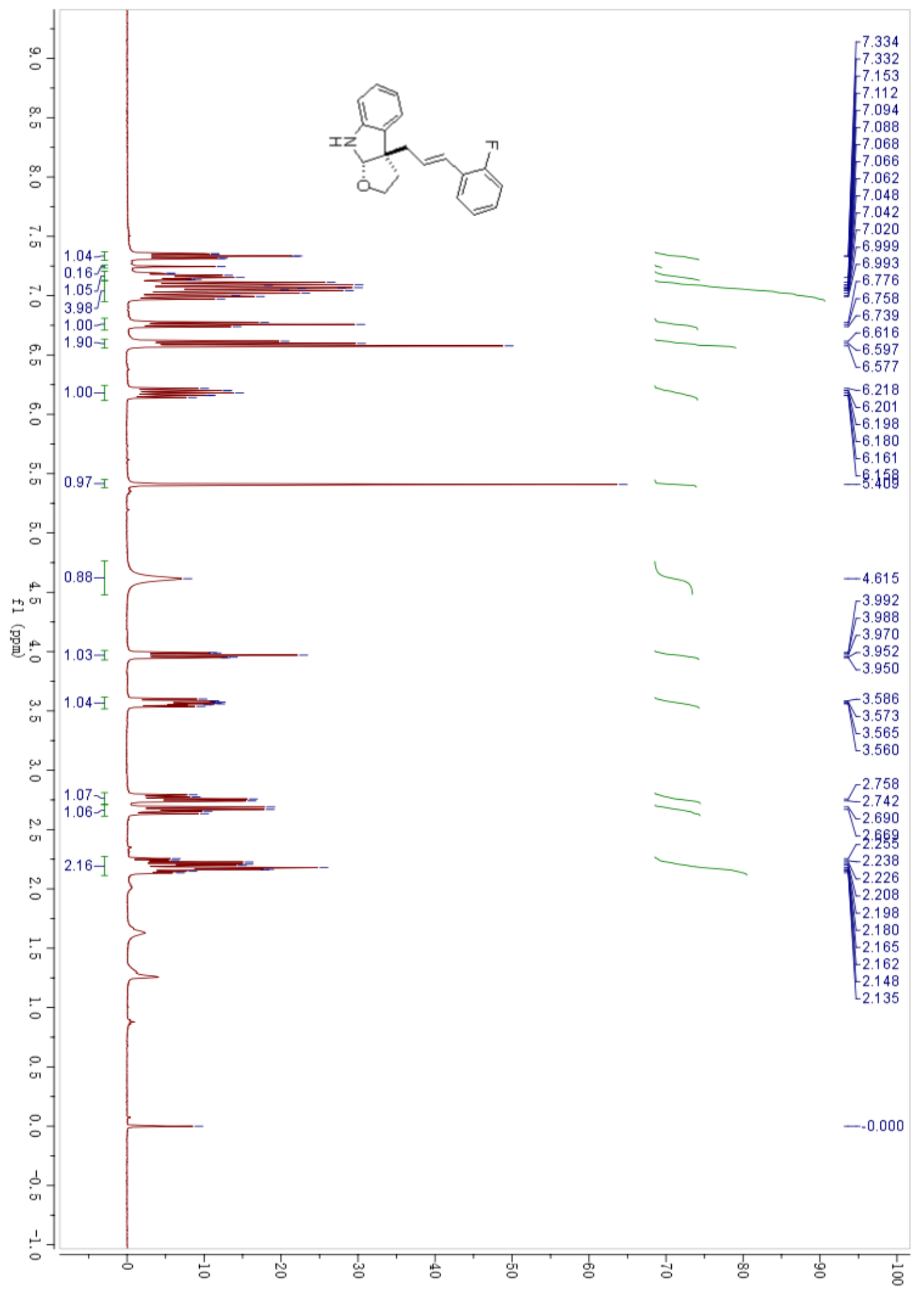


${ }^{13} \mathrm{C}$ NMR spectra of $\mathbf{3 u}$

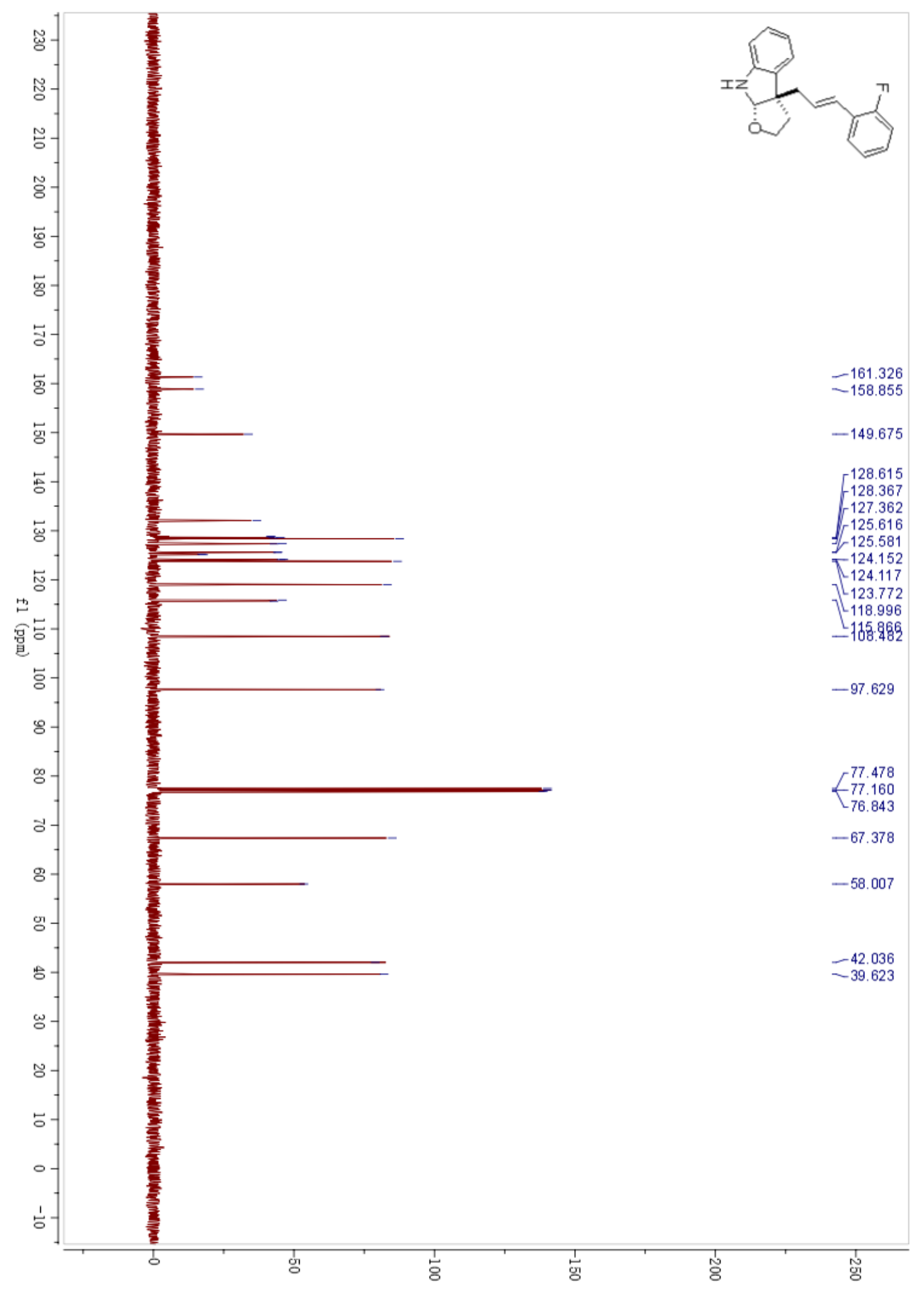


${ }^{19} \mathrm{~F}$ NMR spectra of $\mathbf{3 u}$

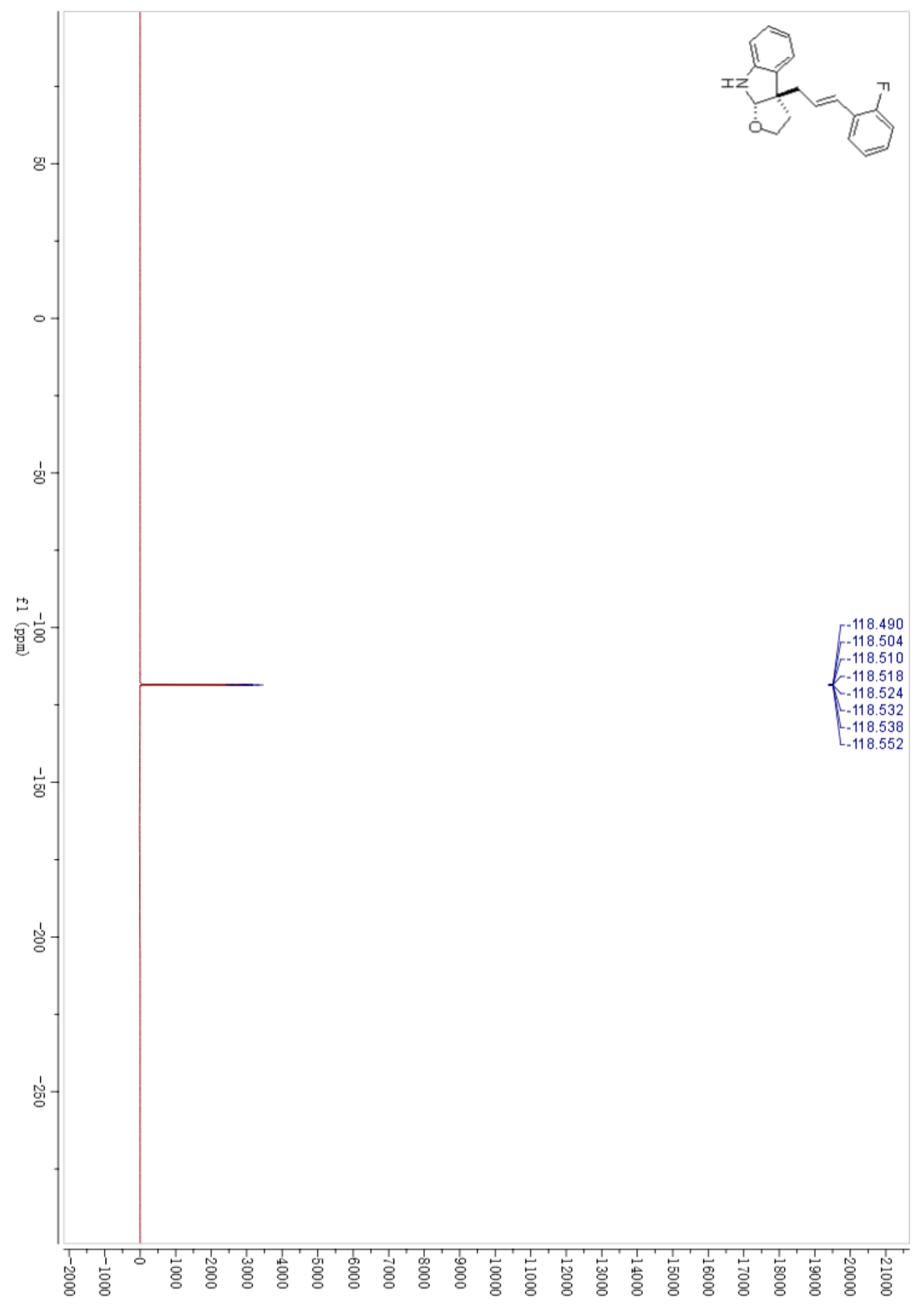


${ }^{1} \mathrm{H}$ NMR spectra of $\mathbf{3 v}$

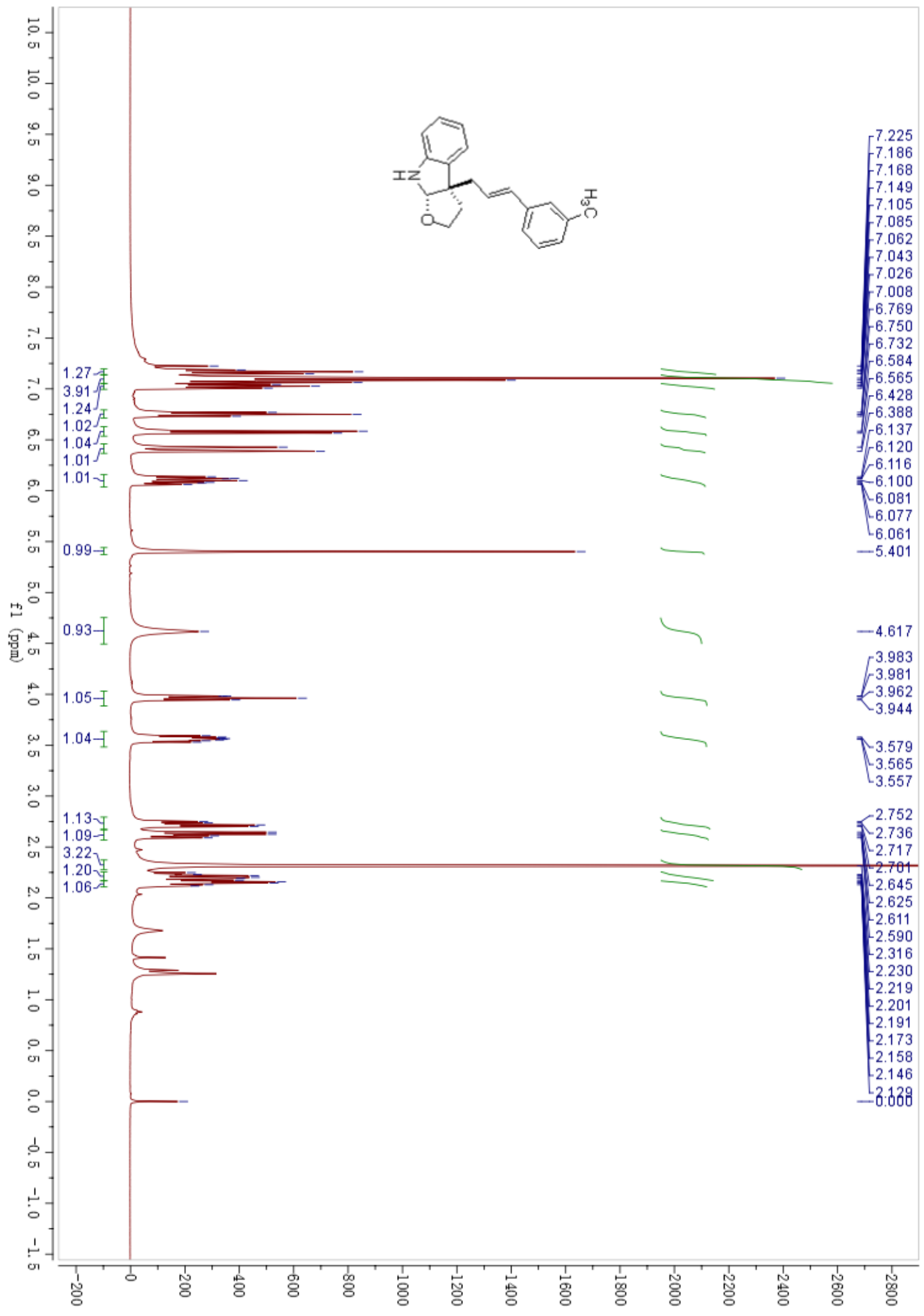


${ }^{13} \mathrm{C}$ NMR spectra of $\mathbf{3 v}$

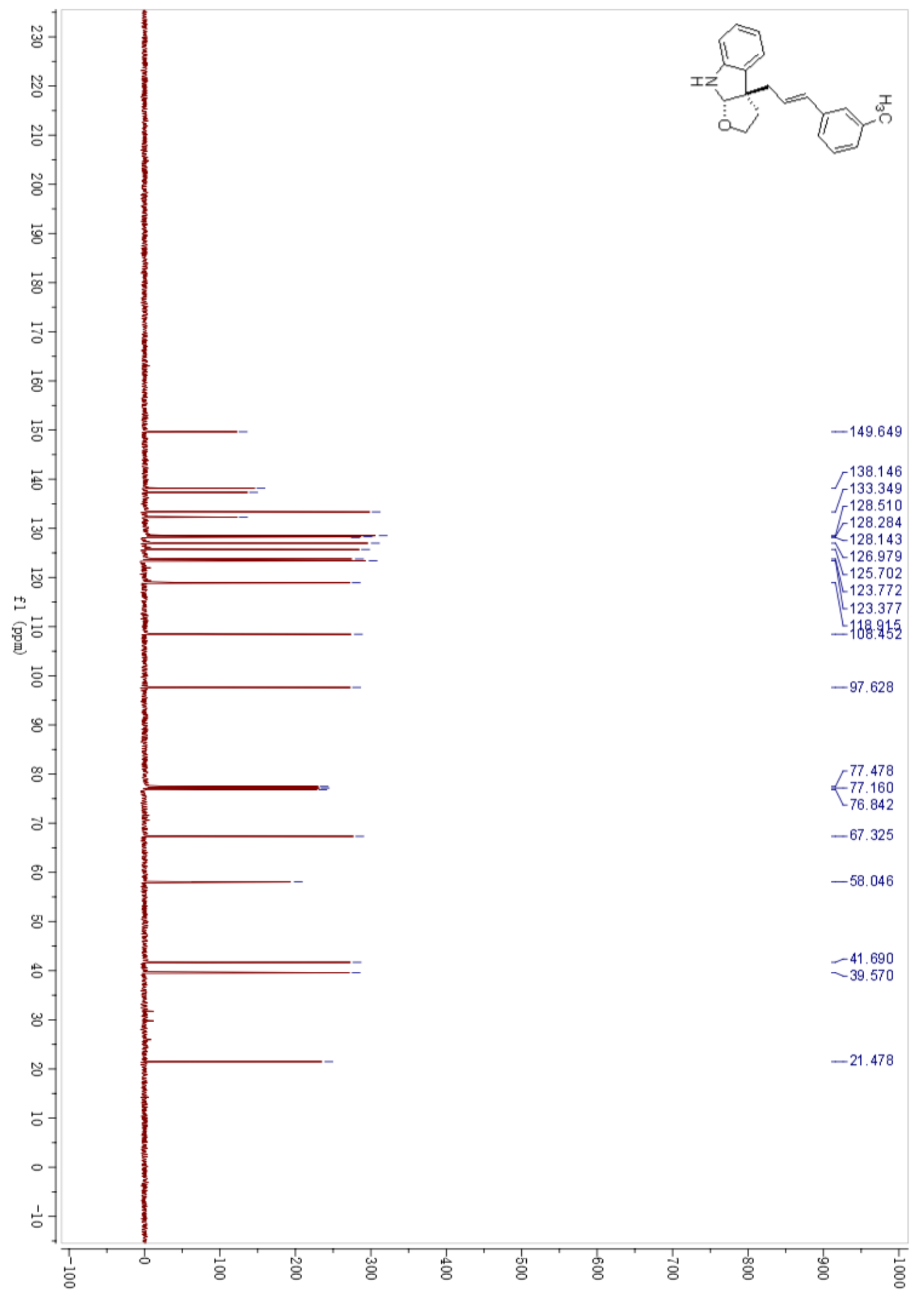


${ }^{1} \mathrm{H}$ NMR spectra of $\mathbf{3 w}$

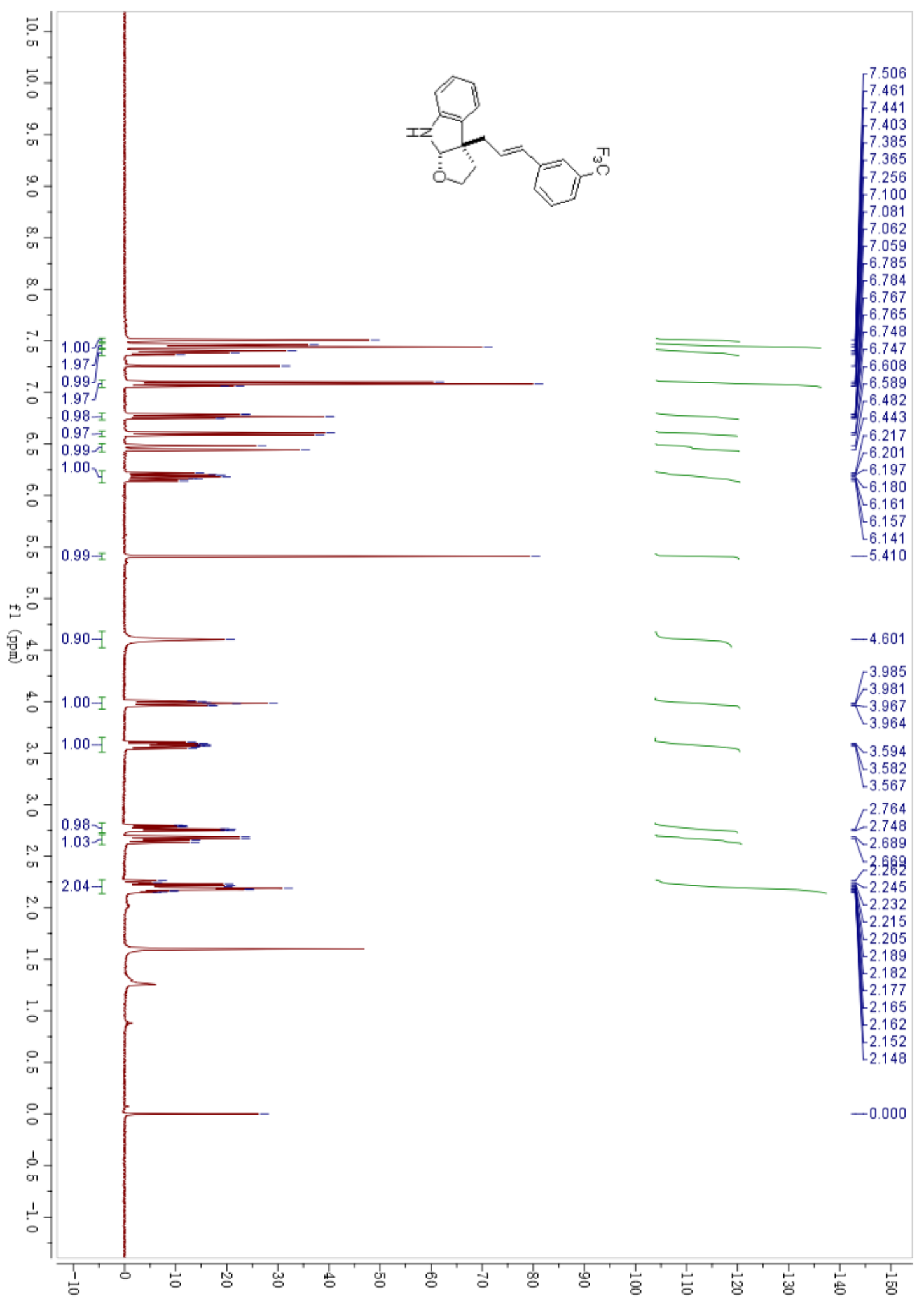

S82 
${ }^{13} \mathrm{C}$ NMR spectra of $\mathbf{3 w}$

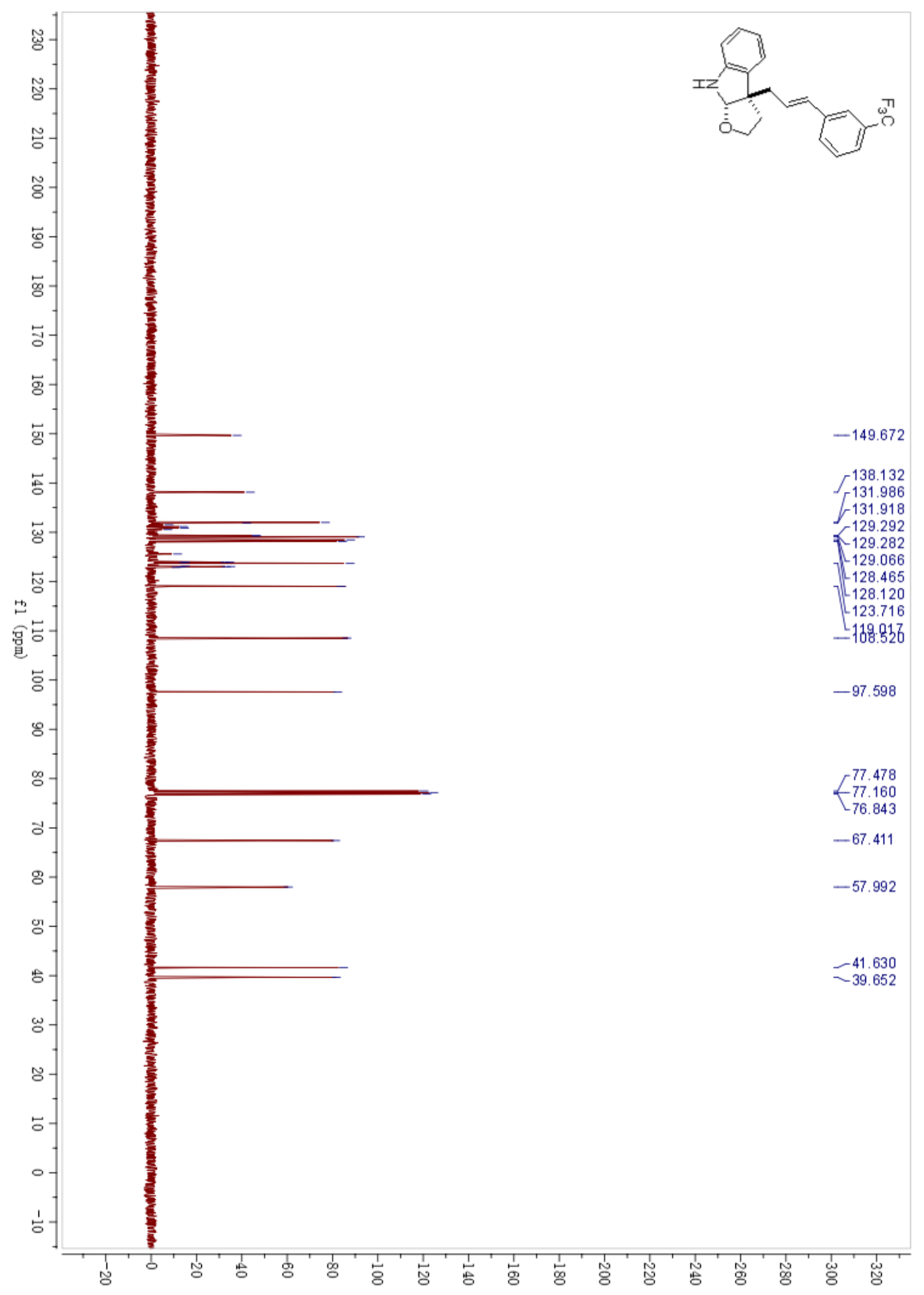


${ }^{19}$ F NMR spectra of $\mathbf{3 w}$

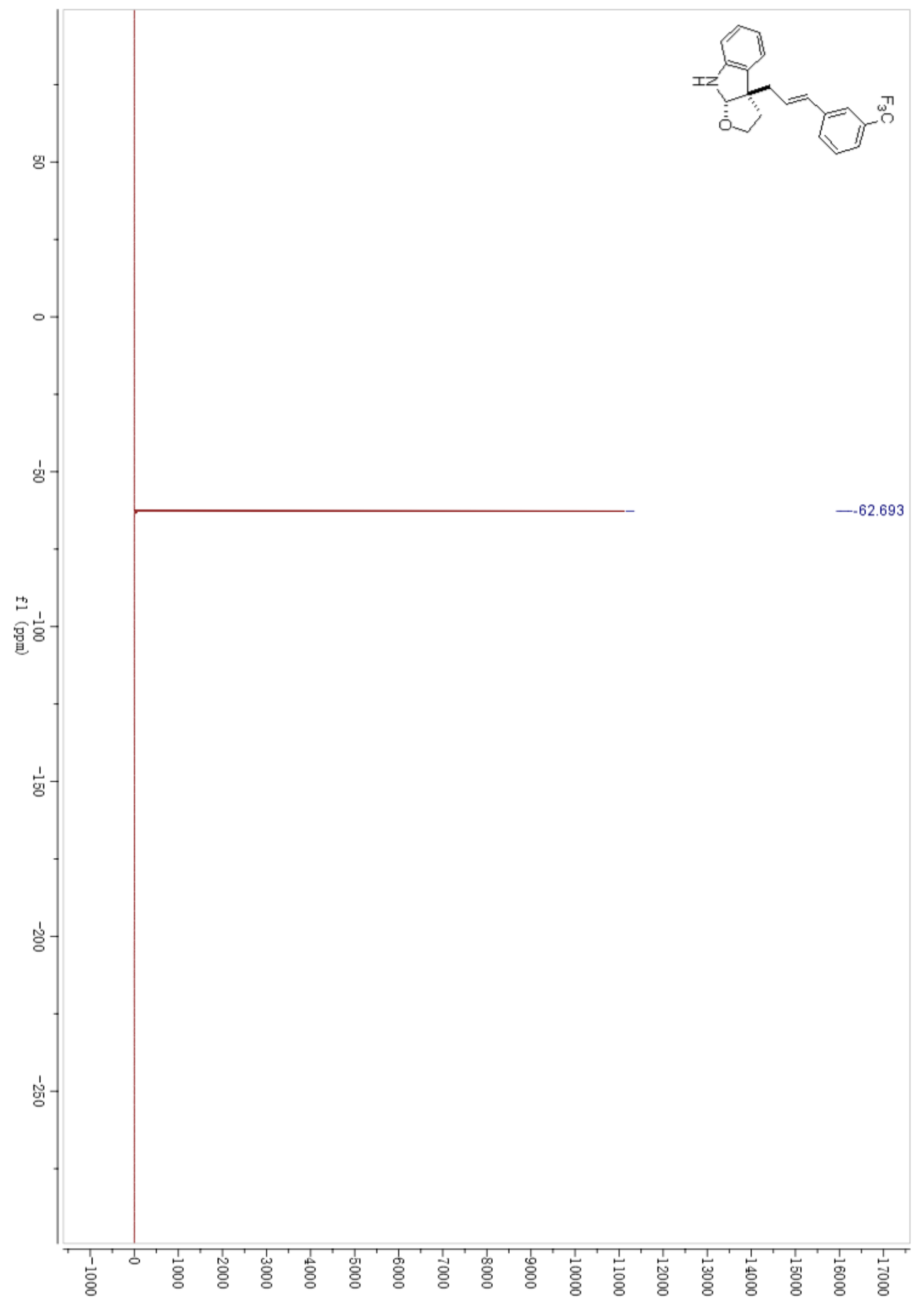


${ }^{1} \mathrm{H}$ NMR spectra of $\mathbf{3 x}$

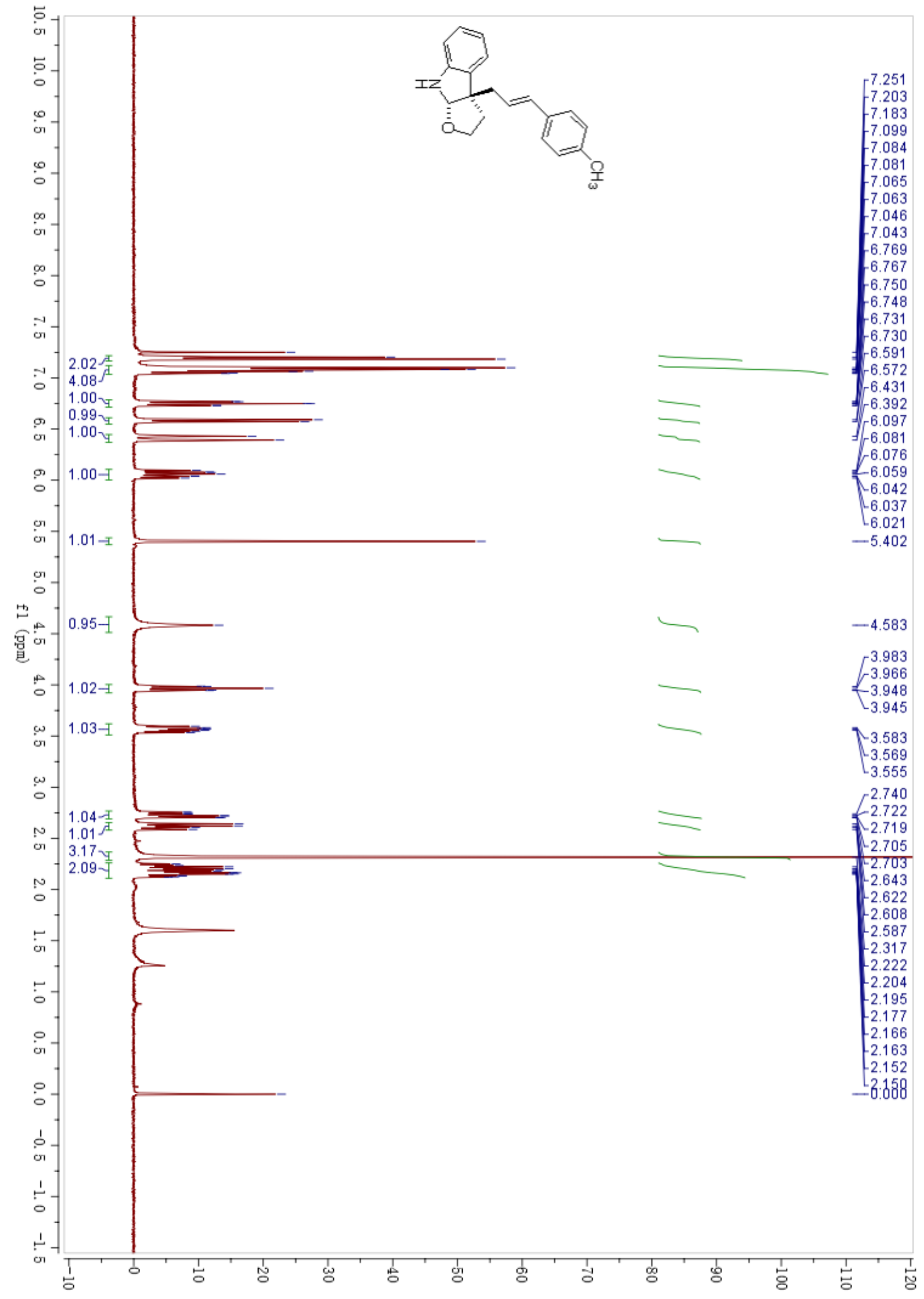

S85 
${ }^{13} \mathrm{C}$ NMR spectra of $\mathbf{3} \mathbf{x}$

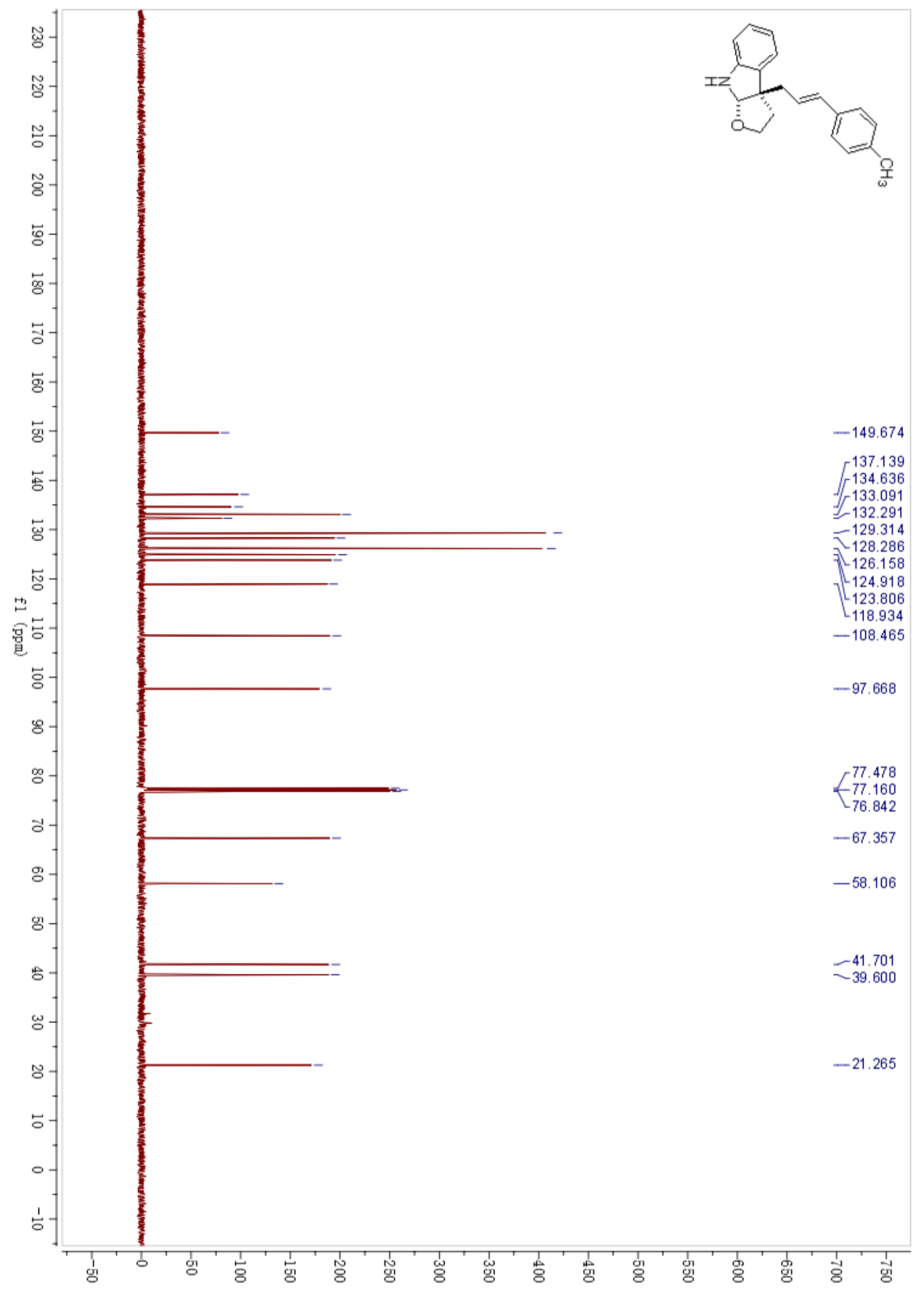


${ }^{1} \mathrm{H}$ NMR spectra of $\mathbf{3 y}$

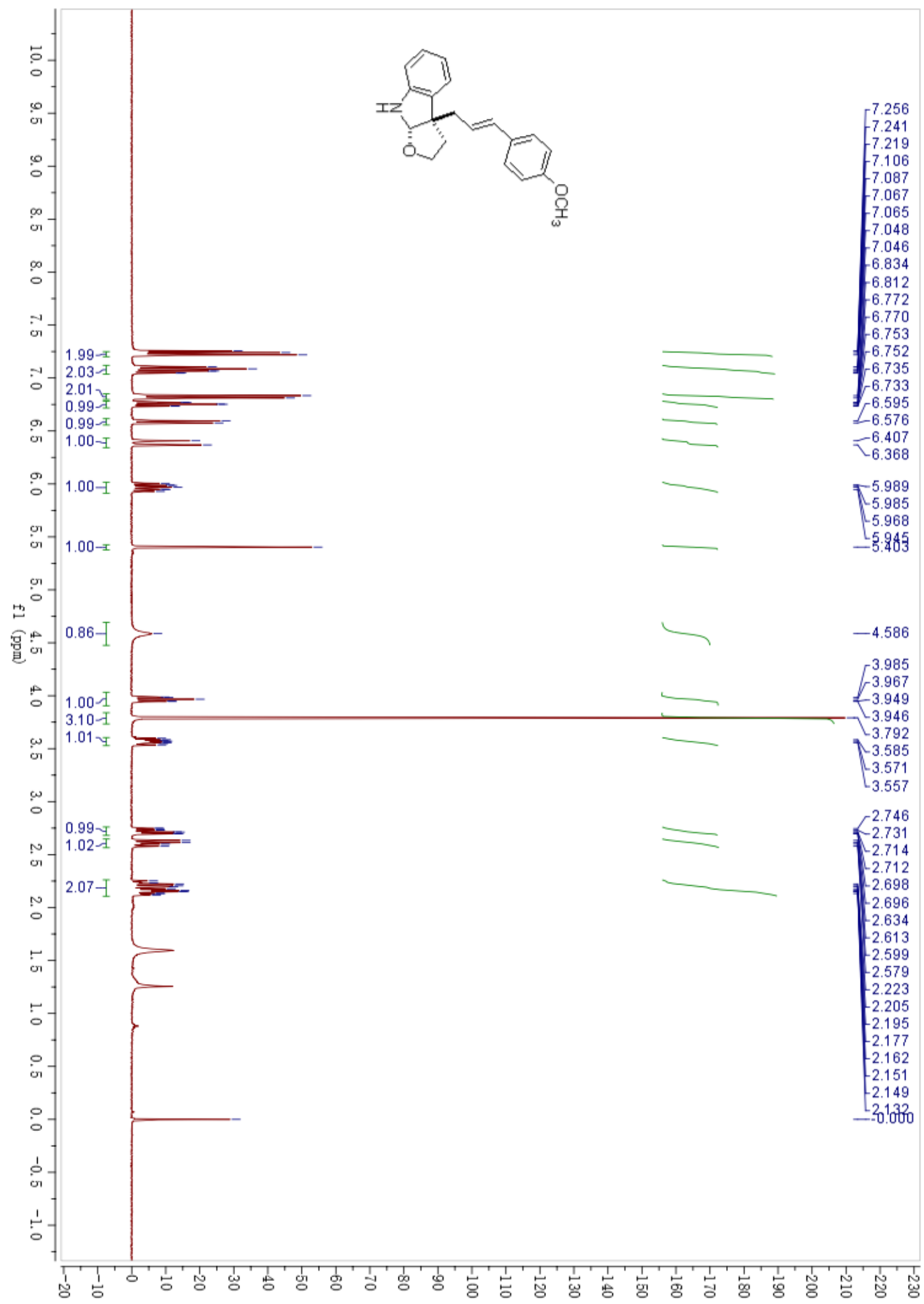


${ }^{13} \mathrm{C}$ NMR spectra of $\mathbf{3 y}$

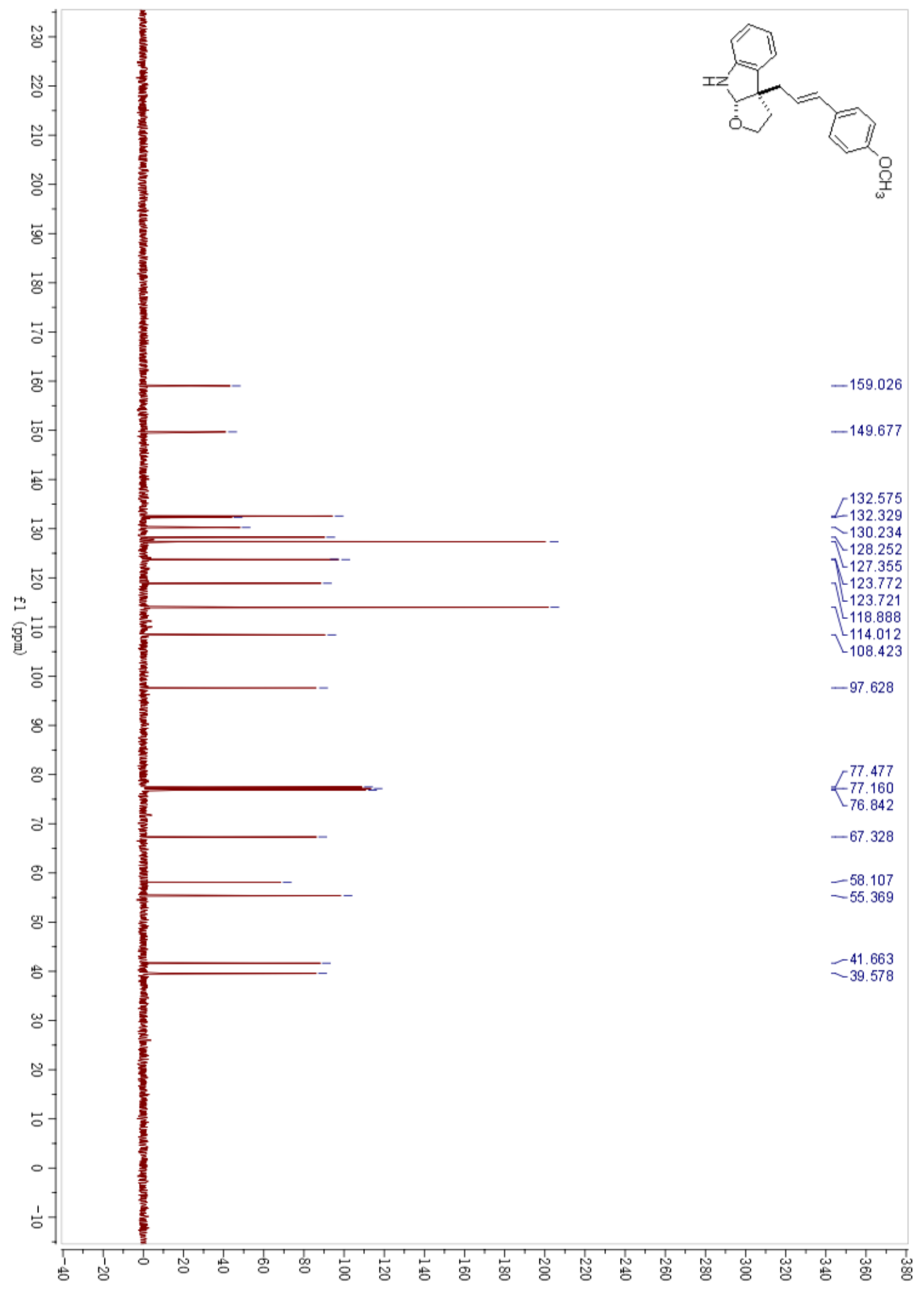


${ }^{1} \mathrm{H}$ NMR spectra of $\mathbf{3 z}$

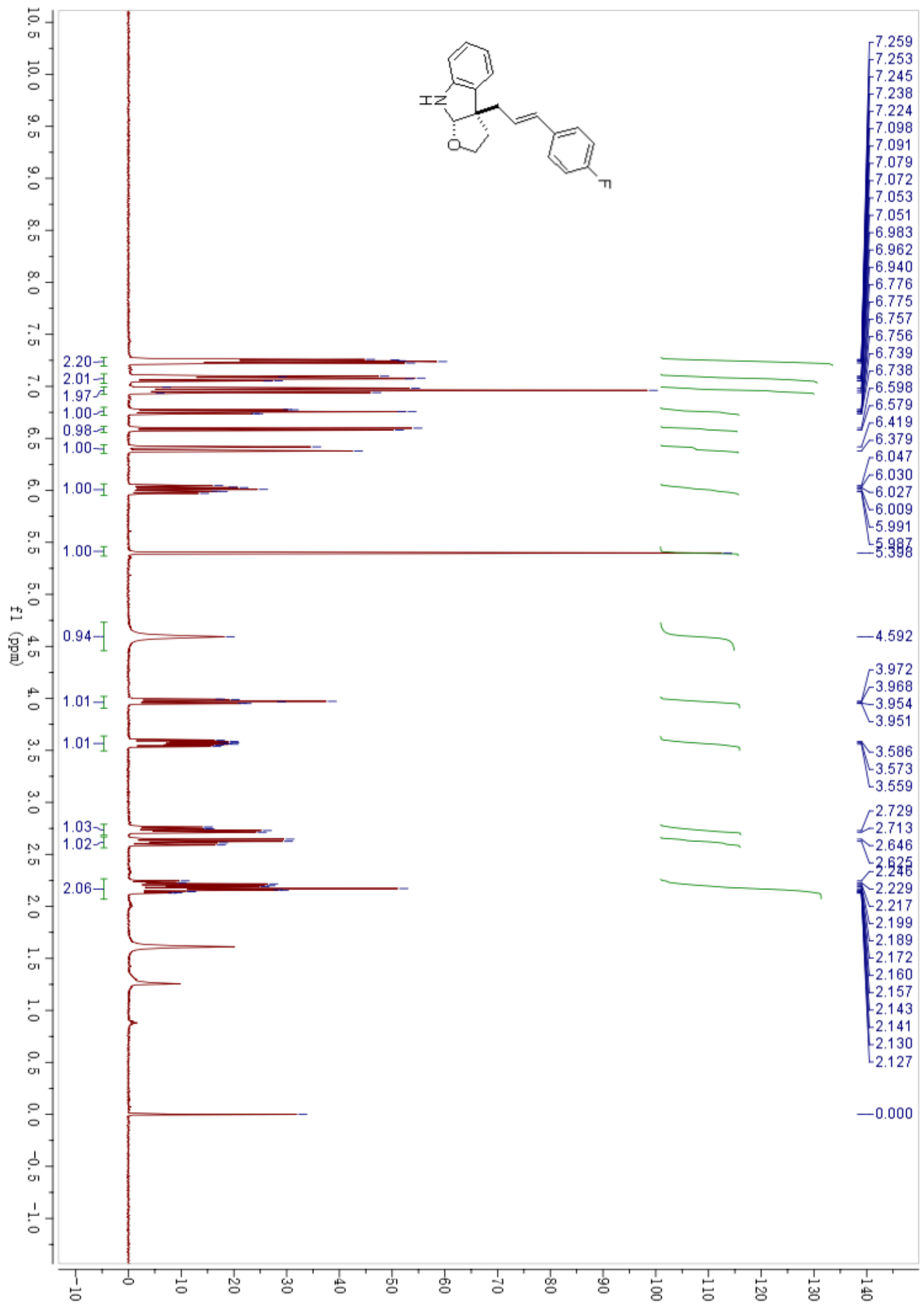


${ }^{13} \mathrm{C}$ NMR spectra of $\mathbf{3 z}$

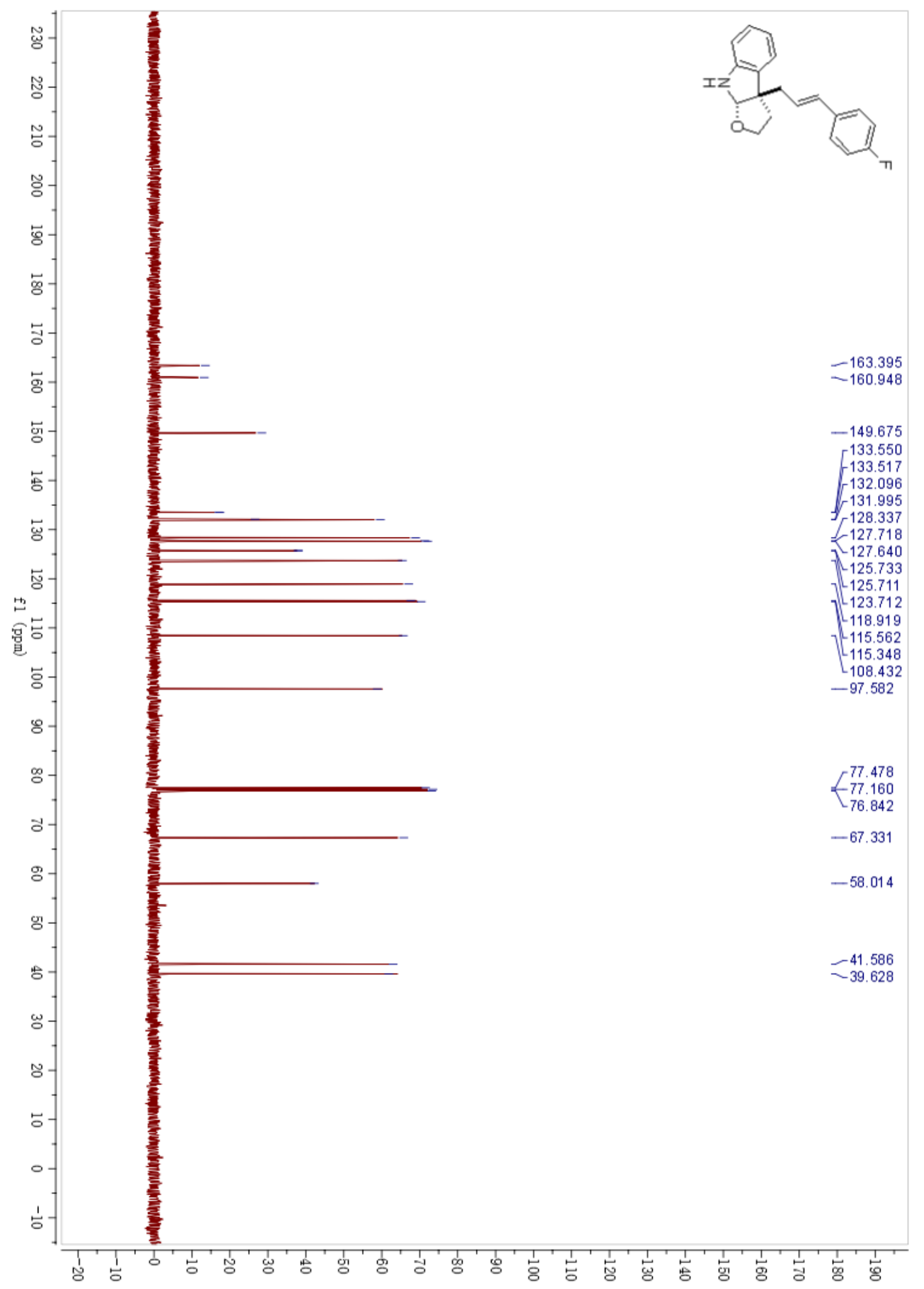


${ }^{19}$ F NMR spectra of $\mathbf{3 z}$

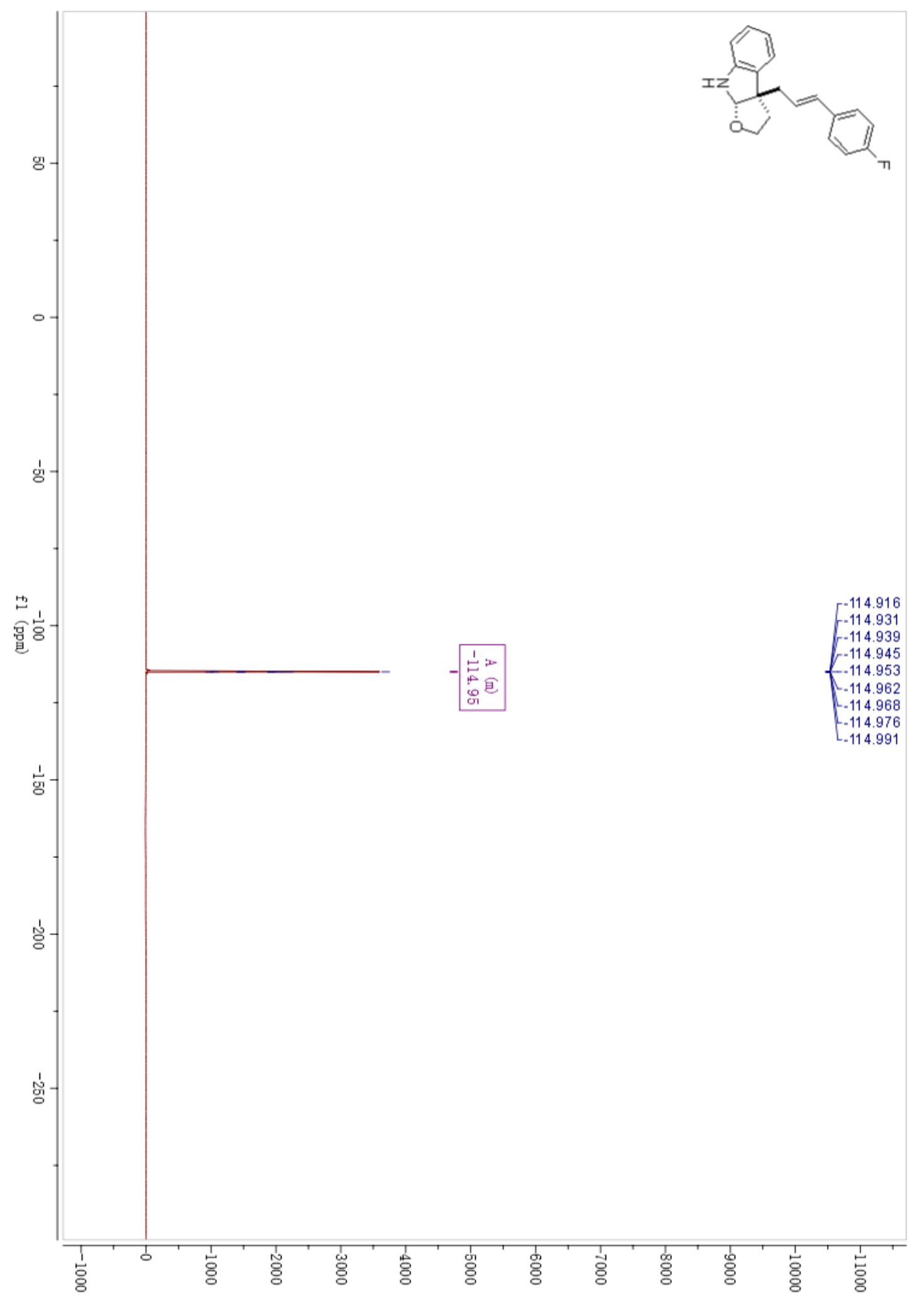


${ }^{1} \mathrm{H}$ NMR spectra of $\mathbf{3 a a}$

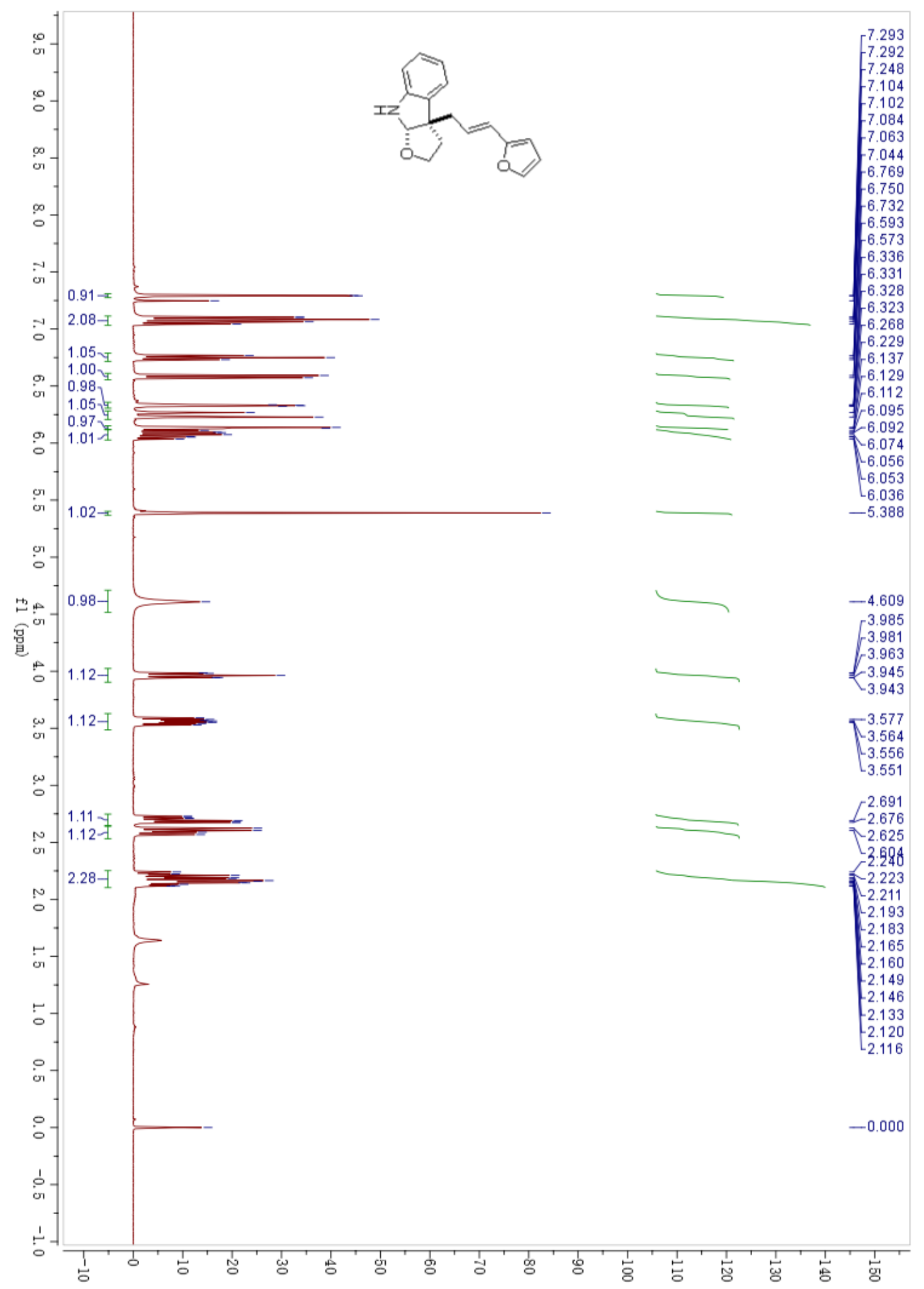


${ }^{13} \mathrm{C}$ NMR spectra of $\mathbf{3 a a}$

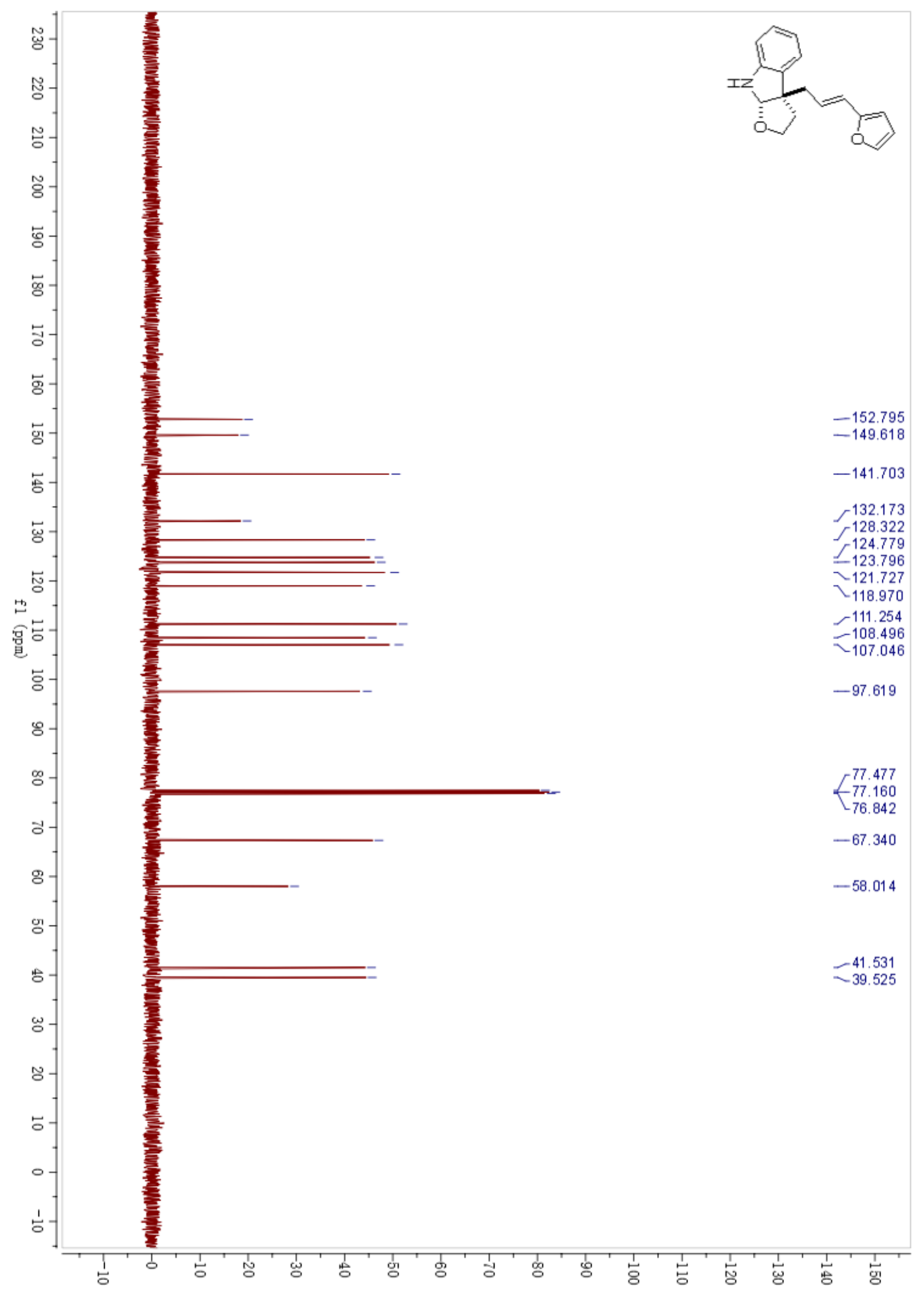


${ }^{1} \mathrm{H}$ NMR spectra of $\mathbf{3 a b}$

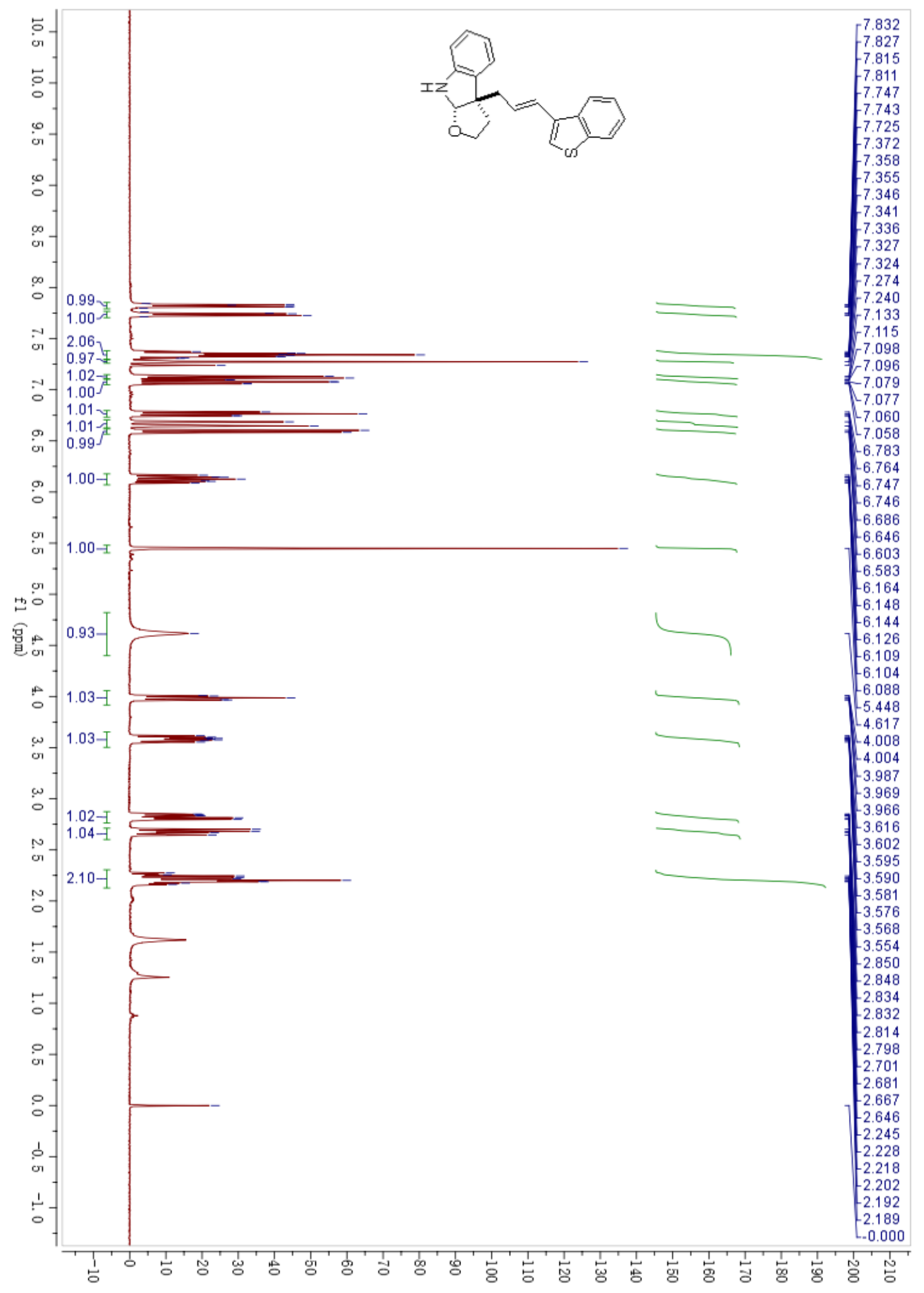


${ }^{13} \mathrm{C}$ NMR spectra of $\mathbf{3 a b}$

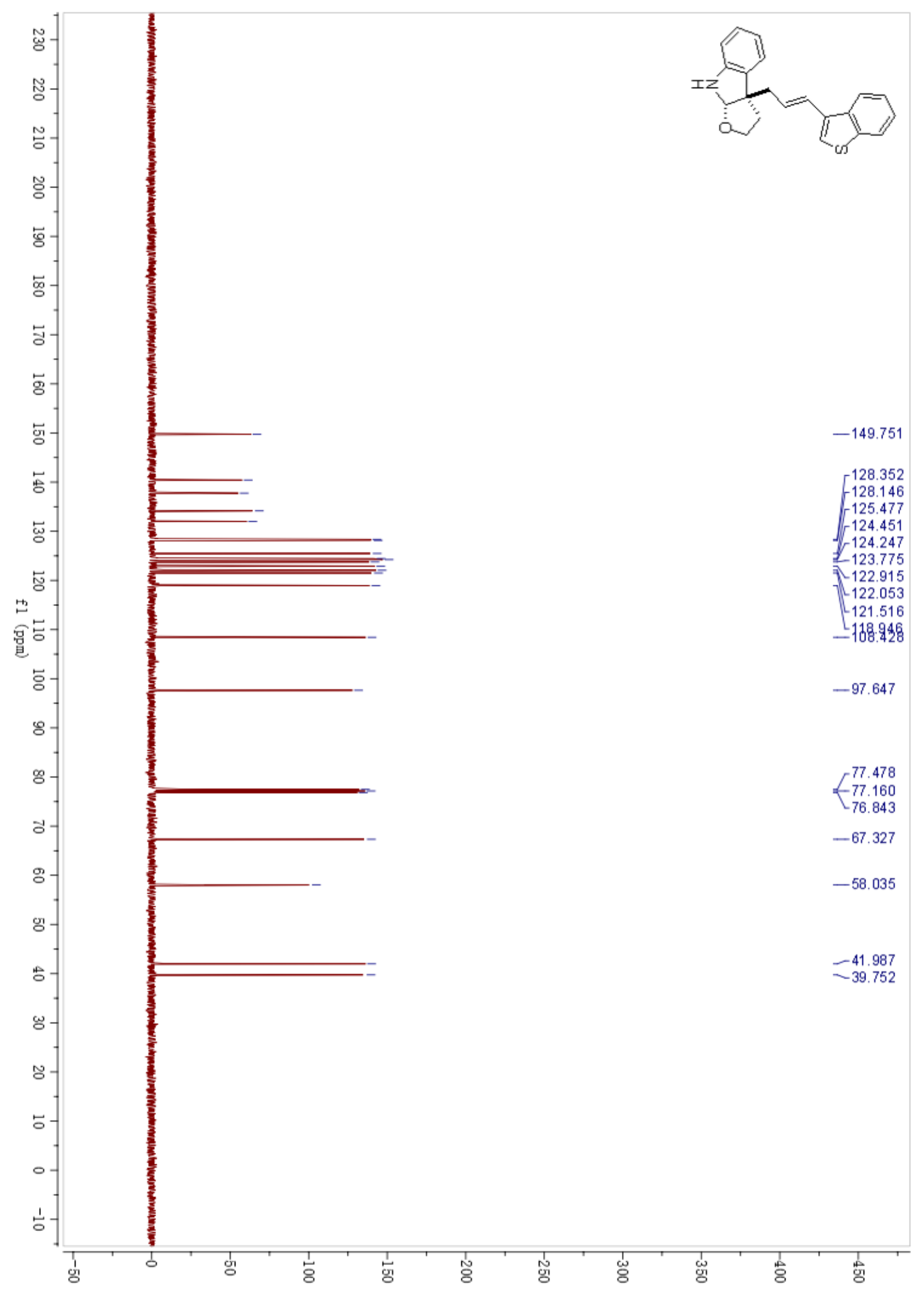


${ }^{1} \mathrm{H}$ NMR spectra of 5

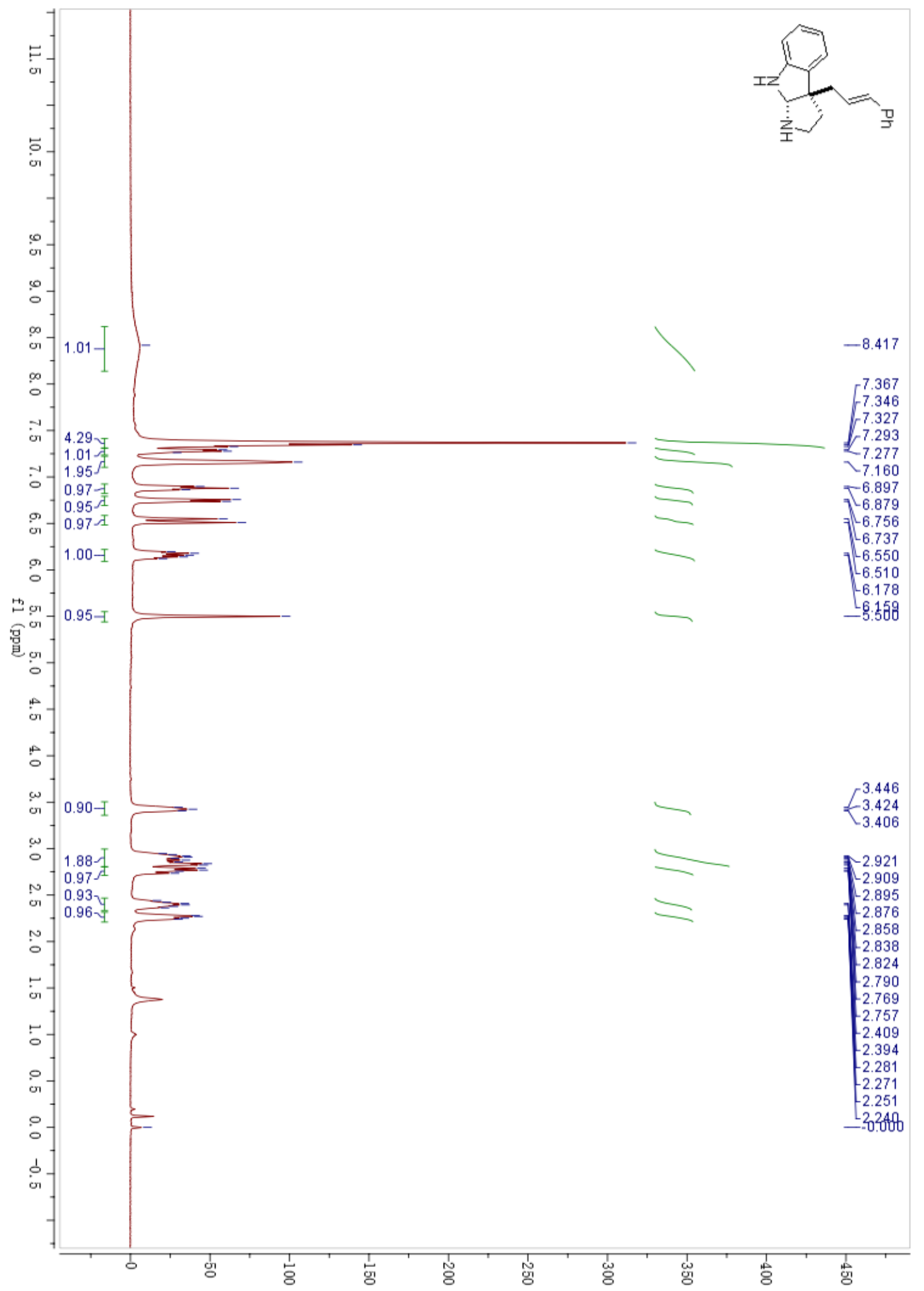


${ }^{13} \mathrm{C}$ NMR spectra of 5

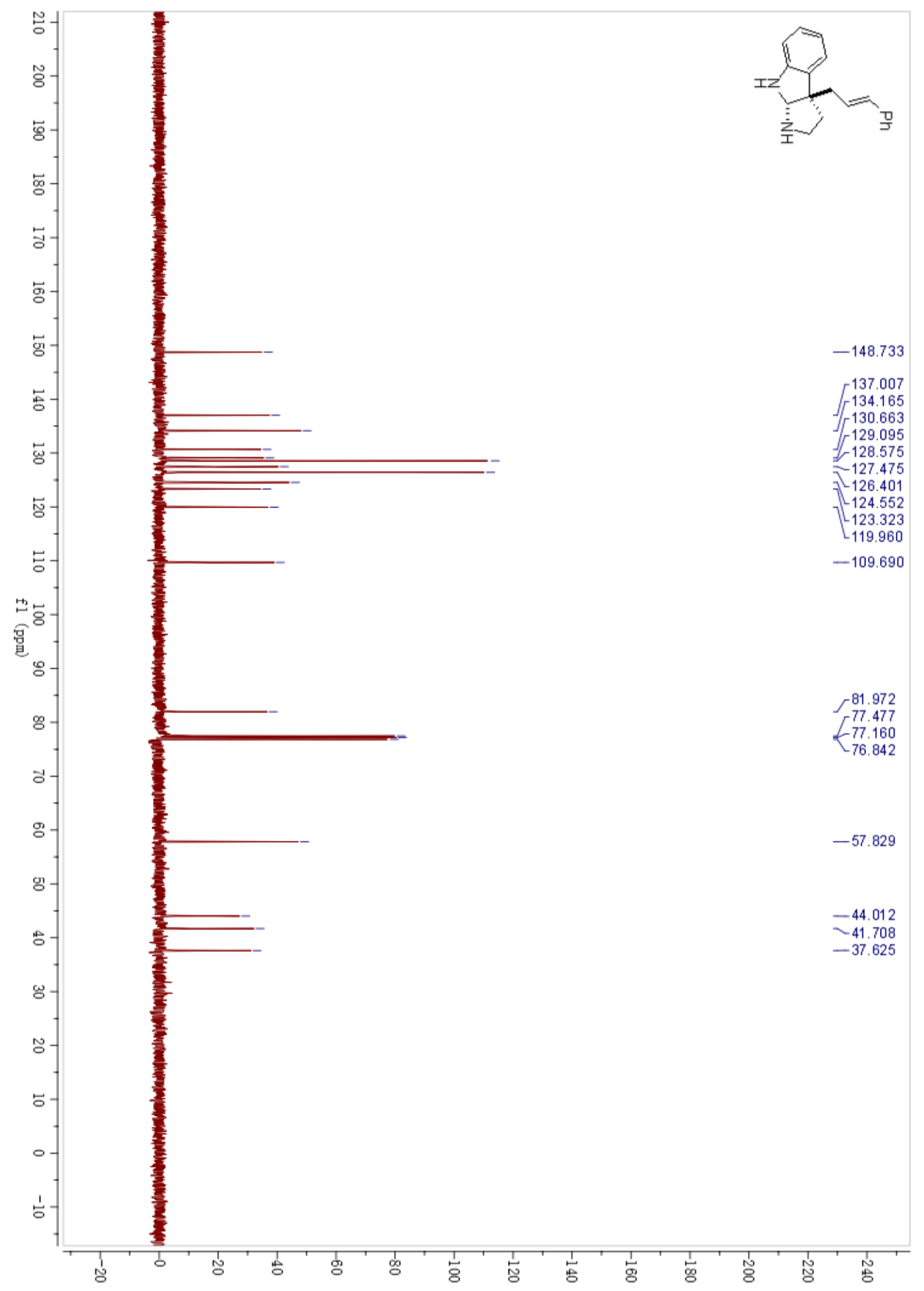


${ }^{1} \mathrm{H}$ NMR spectra of 6

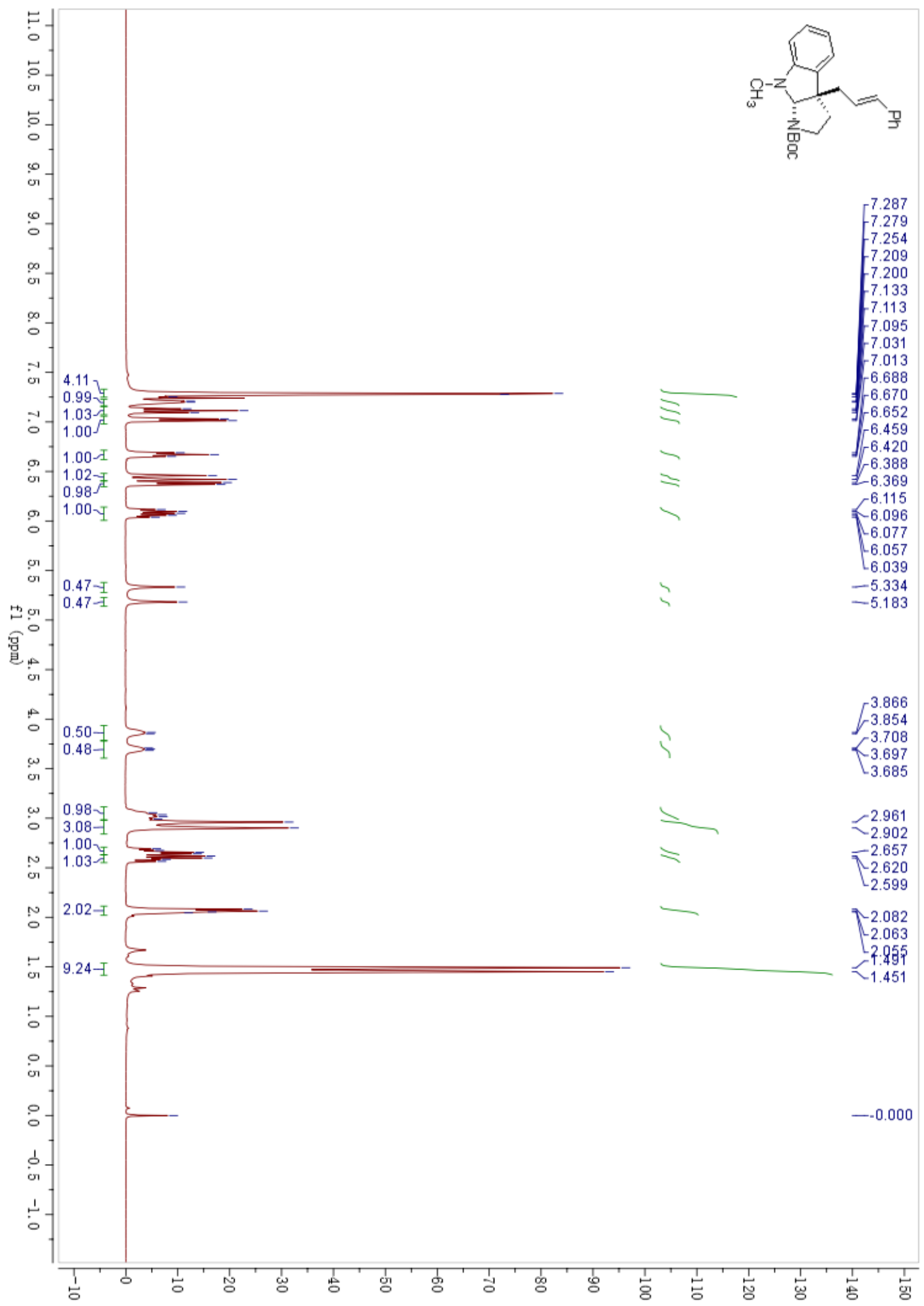


${ }^{13} \mathrm{C}$ NMR spectra of 6

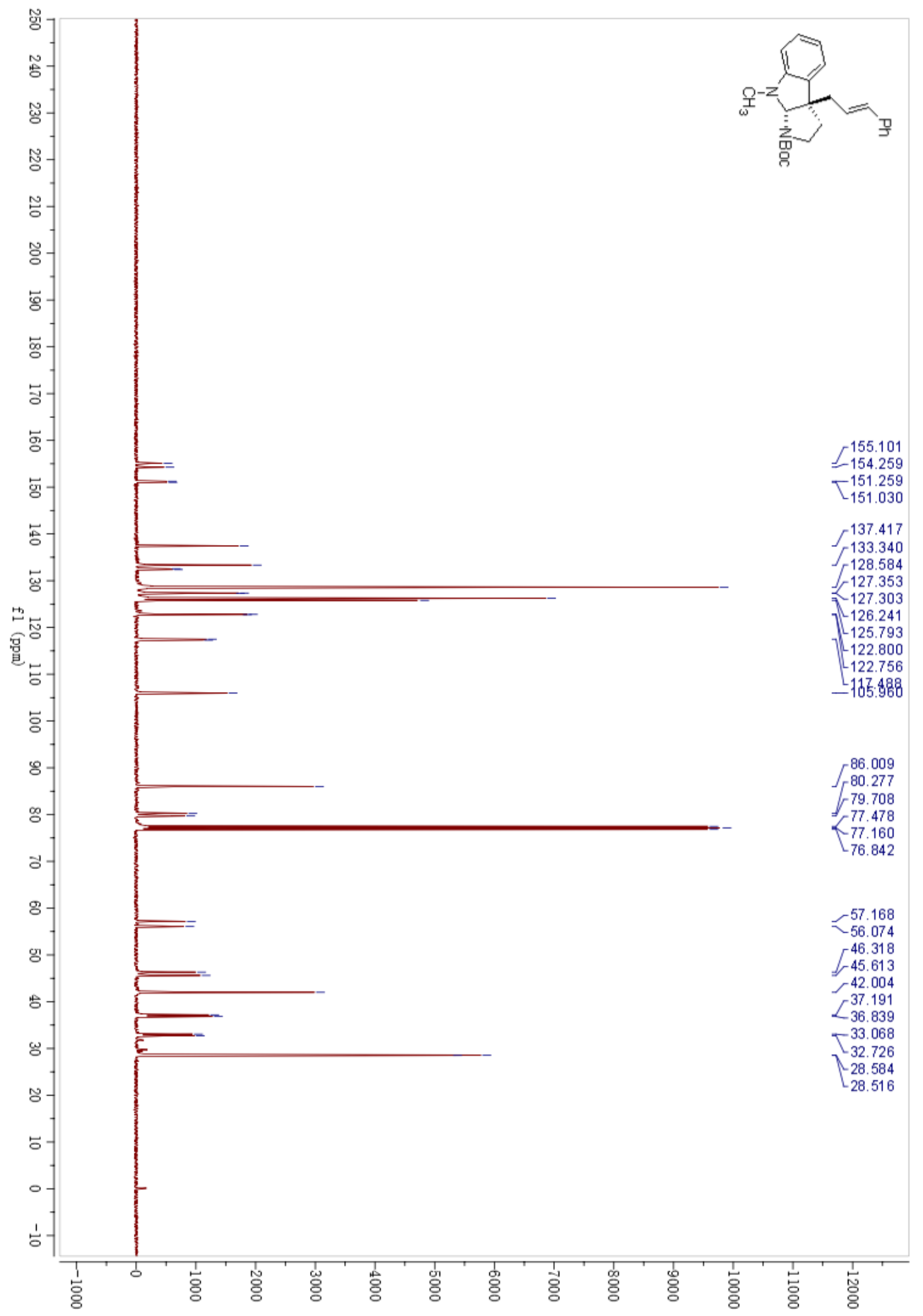


${ }^{1} \mathrm{H}$ NMR spectra of 7

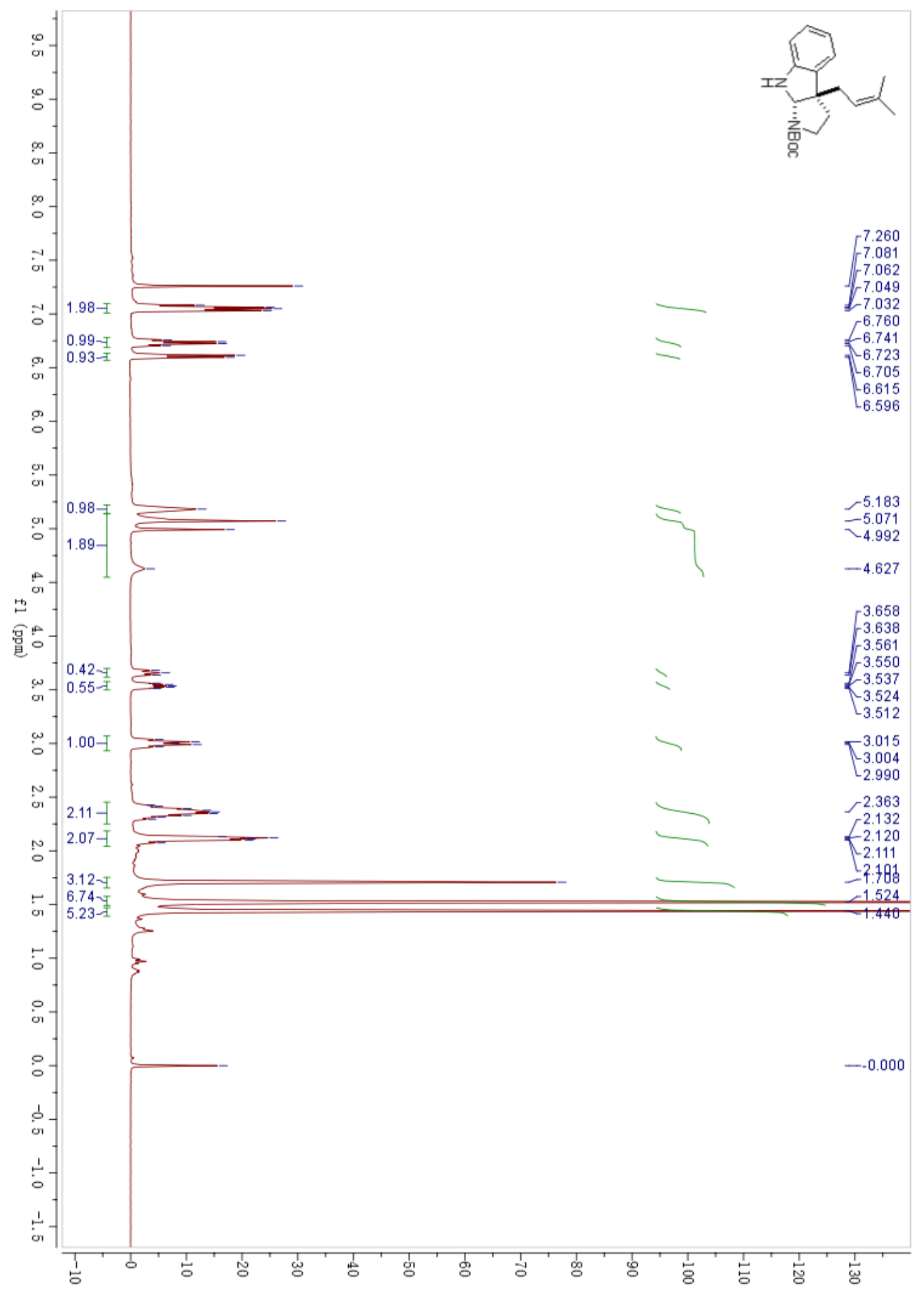


${ }^{13} \mathrm{C}$ NMR spectra of 7

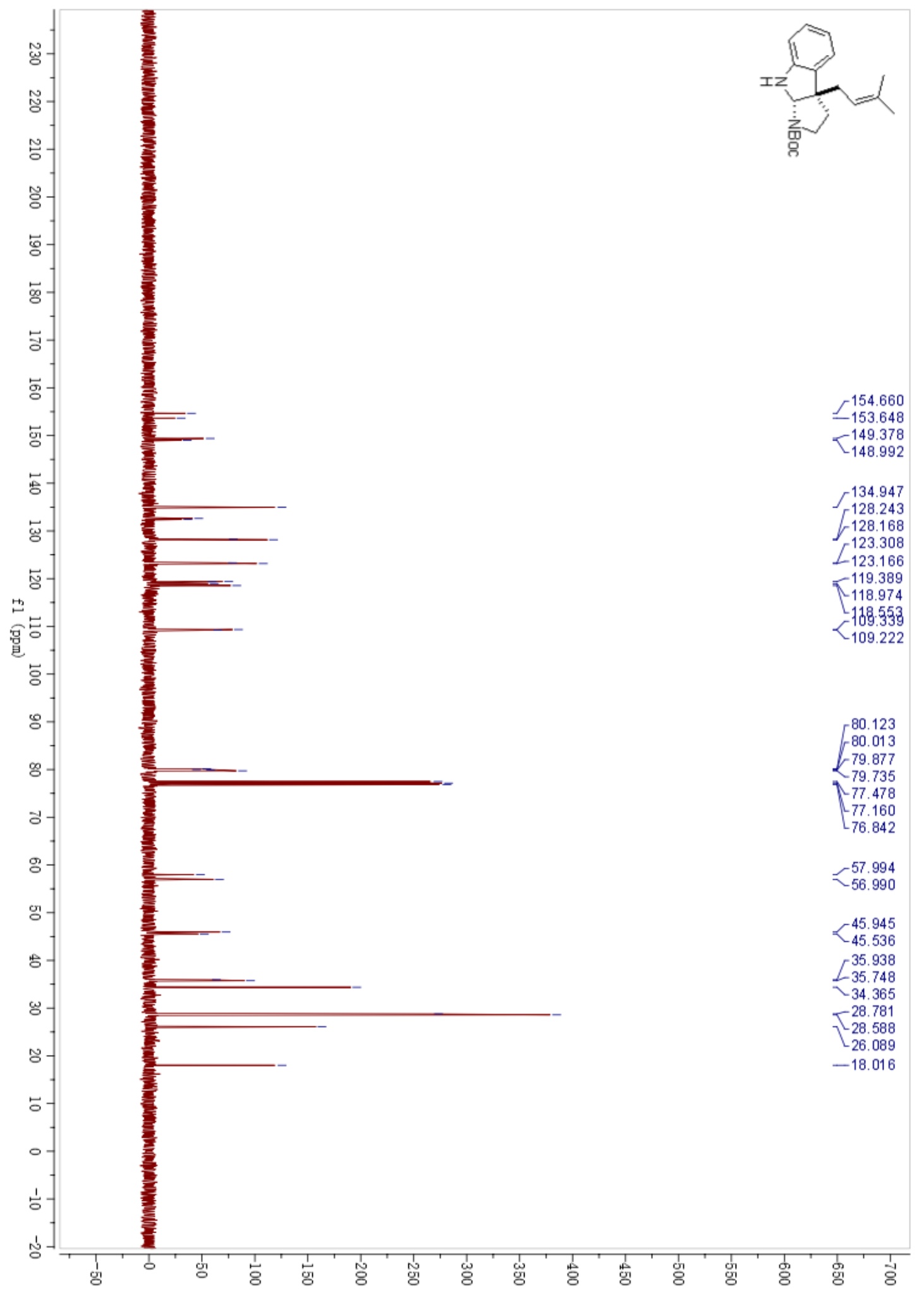




\section{References}

[1] Han, L.; Liu, C.; Zhang, W.; Shi, X.-X.; You, S.-L. Chem. Commun. 2014, 50, 1231.

[2] Tu, D.; Cheng, X.; Gao, Y.; Yang, P.; Ding, Y.; Jiang, C. Org. Biomol. Chem. 2016, 14, 7443.

[3] Lafrance, M.; Roggen, M.; Carreira, E. M. Angew. Chem. Int. Ed. 2012, 51, 3470 . 